\title{
Practical Method for Reductive Deuteration of Ketones with Magnesium and $\mathrm{D}_{2} \mathrm{O}$
}

\begin{abstract}
Nengbo Zhu, Min Su, Wen-Ming Wan, Yajun Li and Hongli Bao*
State Key Laboratory of Structural Chemistry, Key Laboratory of Coal to Ethylene Glycol and Its Related Technology, Center for Excellence in Molecular Synthesis, Fujian Institute of Research on the Structure of Matter, Chinese Academy of Sciences, 155 Yangqiao Road West, Fuzhou, Fujian 350002, P. R. of China.
\end{abstract}


Table of contents:

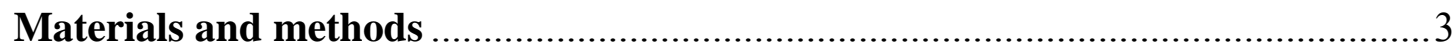

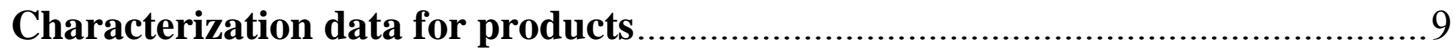

Synthesis of deuterium-labelled drugs and pharmaceutical precursors .............24

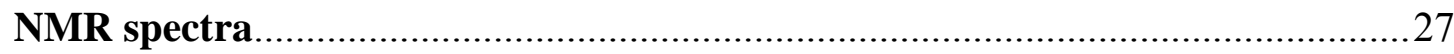

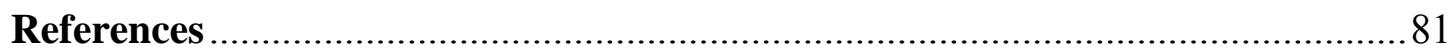




\section{Materials and methods}

All reactions were carried out under an atmosphere of nitrogen in flame-dried glassware with magnetic stirring unless otherwise indicated. Commercially obtained reagents were used as received. Solvents were dried by Innovative Technology Solvent Purification System. Liquids and solutions were transferred via syringe. All reactions were monitored by thin-layer chromatography. ${ }^{1} \mathrm{H},{ }^{13} \mathrm{C}$ and ${ }^{19} \mathrm{~F}$ NMR spectra were recorded on Bruker-BioSpin AVANCE III HD or ECZ 600S. Data for ${ }^{1} \mathrm{H}$ NMR spectra are reported relative to chloroform $(7.26 \mathrm{ppm})$, dimethyl sulfoxide $(3.36 \mathrm{ppm})$ or acetone $(2.05 \mathrm{ppm})$ as an internal standard and are reported as follows: chemical shift (ppm), multiplicity, coupling constant $(\mathrm{Hz})$, and integration. Data for ${ }^{13} \mathrm{C} N M R$ spectra are reported relative to chloroform $(77.23 \mathrm{ppm})$, dimethyl sulfoxide (39.98 ppm) or acetone (29.84 ppm) as an internal standard and are reported in terms of chemical shift (ppm). HRMS data were recorded on Bruker Impact II UHR-TOF or Waters Micromass GCT Premier. Melting point data were recorded on INESA-SGW $\mathrm{X}-4$. IR data $(\mathrm{KBr})$ were recorded on Bruker Vertex 70.

\section{Synthesis of ketones}

The following ketones were synthesized by references or corresponding method. Other ketones were commercially available.

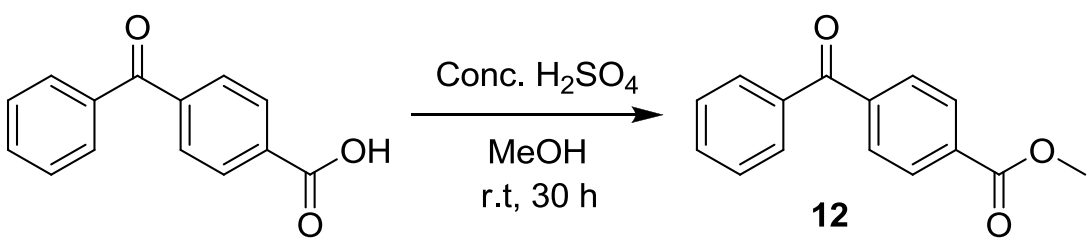

The compound was synthesized according to literature. ${ }^{1}$ 4-Benzoylbenzoic acid (5.0 mmol, $1130 \mathrm{mg})$, Conc. $\mathrm{H}_{2} \mathrm{SO}_{4}(0.53 \mathrm{~mL})$ and $\mathrm{MeOH}(50 \mathrm{~mL})$ were added into a round button flask with a stirring bar and stirred at room temperature for $30 \mathrm{~h}$. The solution was concentrated under reduced pressure and workup with dichloromethane/water, the organic solution was dried with anhydrous $\mathrm{MgSO}_{4}$ and concentrated under reduced pressure and the residue was purified by column chromatography on silica gel (PE/EA $=20 / 1-3 / 1)$ to give the product as a white solid (600 mg, $50 \%$ yield). ${ }^{1} \mathrm{H}$ NMR $\left(400 \mathrm{MHz}, \mathrm{CDCl}_{3}\right) \delta 8.15(\mathrm{~d}, J=8.2 \mathrm{~Hz}, 2 \mathrm{H}), 7.87-$ $7.78(\mathrm{~m}, 4 \mathrm{H}), 7.62(\mathrm{t}, J=7.4 \mathrm{~Hz}, 1 \mathrm{H}), 7.50(\mathrm{t}, J=7.6 \mathrm{~Hz}, 2 \mathrm{H}), 3.96(\mathrm{~s}, 3 \mathrm{H}) .{ }^{13} \mathrm{C} \mathrm{NMR}$ $\left(100 \mathrm{MHz}, \mathrm{CDCl}_{3}\right) \delta 196.0,166.3,141.3,136.9,133.2,133.0,130.1,129.8,129.5$, $128.5,52.5$. The data matches with the reported value.<smiles>C=Cc1ccc(C(=O)c2ccccc2)cc1</smiles> 
The compound was synthesized according to literature. ${ }^{2}{ }^{1} \mathrm{H}$ NMR $\left(400 \mathrm{MHz}, \mathrm{CDCl}_{3}\right)$ $\delta 7.83-7.73(\mathrm{~m}, 4 \mathrm{H}), 7.57(\mathrm{~d}, J=7.4 \mathrm{~Hz}, 1 \mathrm{H}), 7.52-7.41(\mathrm{~m}, 4 \mathrm{H}), 6.77(\mathrm{dd}, J=$ 17.6, $10.9 \mathrm{~Hz}, 1 \mathrm{H}), 5.88(\mathrm{~d}, J=17.6 \mathrm{~Hz}, 1 \mathrm{H}), 5.40(\mathrm{~d}, J=10.9 \mathrm{~Hz}, 1 \mathrm{H}) .{ }^{13} \mathrm{C}$ NMR $\left(100 \mathrm{MHz}, \mathrm{CDCl}_{3}\right) \delta 196.2,141.6,137.7,136.7,136.0,132.4,130.6,123.0,128.3$, 126.1, 116.6. The data matches with the reported value.<smiles>O=C(c1ccccc1)c1ccc2c(c1)OCO2</smiles>

The compound was synthesized according to literature. ${ }^{3} \mathrm{H}$ NMR (400 $\mathrm{MHz}, \mathrm{CDCl}_{3}$ ) $\delta 7.74(\mathrm{~d}, J=7.3 \mathrm{~Hz}, 2 \mathrm{H}), 7.56(\mathrm{~d}, J=7.5 \mathrm{~Hz}, 1 \mathrm{H}), 7.47(\mathrm{t}, J=7.6 \mathrm{~Hz}, 2 \mathrm{H}), 7.37(\mathrm{~d}, J$ $=7.7 \mathrm{~Hz}, 2 \mathrm{H}), 6.86(\mathrm{~d}, J=7.8 \mathrm{~Hz}, 1 \mathrm{H}), 6.06(\mathrm{~s}, 2 \mathrm{H}) .{ }^{13} \mathrm{C} \mathrm{NMR}\left(100 \mathrm{MHz}, \mathrm{CDCl}_{3}\right) \delta$ 195.1, 151.5, 147.9, 138.1, 132.0, 131.9, 129.7, 128.2, 126.9, 109.9, 107.7, 101.9.. The data matches with the reported value. 
Table S1. Reaction Condition Screening ${ }^{a}$.<smiles>O=C(c1ccccc1)c1ccccc1</smiles>

1

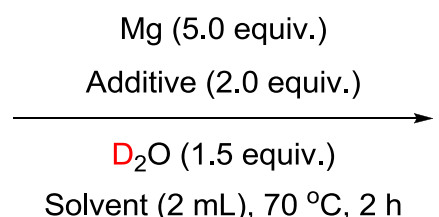

Solvent $(2 \mathrm{~mL}), 70^{\circ} \mathrm{C}, 2 \mathrm{~h}$<smiles>[2H]C(O)(c1ccccc1)c1ccccc1</smiles>

$1 a$<smiles>OC(c1ccccc1)(c1ccccc1)C(O)(c1ccccc1)c1ccccc1</smiles>

$1 \mathrm{~b}$

\begin{tabular}{|c|c|c|c|c|}
\hline Entry & Additive & Solvent & Yield of 1a & Yield of $\mathbf{1 b}$ \\
\hline $1^{\mathrm{b}}$ & $\mathrm{LiCl}$ & THF & Trace & Trace \\
\hline 2 & $\mathrm{MgI}_{2}$ & THF & Trace & Trace \\
\hline $3^{\mathrm{b}}$ & $\mathrm{BrCH}_{2} \mathrm{Br}$ & THF & $7 \%(40 \% \mathrm{D})^{\mathrm{c}}$ & Trace \\
\hline 4 & $\mathrm{BrCH}_{2} \mathrm{CH}_{2} \mathrm{Br}$ & THF & $83 \%(>98 \% \mathrm{D})^{\mathrm{c}}$ & $12 \%$ \\
\hline 5 & $\mathrm{BrCH}_{2} \mathrm{CH}_{3}$ & THF & $30 \%(27 \% \mathrm{D})^{\mathrm{c}}$ & $45 \%$ \\
\hline $6^{\mathrm{b}}$ & $\mathrm{Br}\left(\mathrm{CH}_{2}\right)_{3} \mathrm{Br}$ & THF & $2 \%(74 \% \mathrm{D})^{\mathrm{c}}$ & Trace \\
\hline $7^{\mathrm{b}}$ & $\mathrm{BrCH}_{2} \mathrm{CH}_{2} \mathrm{CH}_{3}$ & THF & Trace & Trace \\
\hline 8 & $\mathrm{Br}\left(\mathrm{CH}_{2}\right)_{4} \mathrm{Br}$ & THF & $30 \%(<2 \% \mathrm{D})^{\mathrm{c}}$ & $35 \%$ \\
\hline 9 & $\mathrm{Br}\left(\mathrm{CH}_{2}\right)_{5} \mathrm{Br}$ & THF & $57(<2 \% \mathrm{D})^{\mathrm{c}}$ & $16 \%$ \\
\hline 10 & $\mathrm{Br}\left(\mathrm{CH}_{2}\right)_{6} \mathrm{Br}$ & THF & $68 \%(<2 \% \mathrm{D})^{\mathrm{c}}$ & $15 \%$ \\
\hline 11 & $\mathrm{BrCH}_{2} \mathrm{CH}_{2} \mathrm{Br}$ & Ether & $15 \%$ & Trace \\
\hline $12^{\mathrm{b}}$ & $\mathrm{BrCH}_{2} \mathrm{CH}_{2} \mathrm{Br}$ & MTBE & Trace & Trace \\
\hline $13^{\mathrm{b}}$ & $\mathrm{BrCH}_{2} \mathrm{CH}_{2} \mathrm{Br}$ & Dioxane & Trace & Trace \\
\hline $14^{\mathrm{b}}$ & $\mathrm{BrCH}_{2} \mathrm{CH}_{2} \mathrm{Br}$ & DME & Trace & Trace \\
\hline $15^{\mathrm{d}}$ & $\mathrm{BrCH}_{2} \mathrm{CH}_{2} \mathrm{Br}$ & THF & Trace & Trace \\
\hline $16^{\mathrm{b}}$ & -- & THF & Trace & $4 \%$ \\
\hline
\end{tabular}

a. Conditions: benzophenone (1.0 mmol), additive (2.0 equiv.), $\mathrm{Mg}$ (freshly peeled, 5.0 equiv.), and $\mathrm{D}_{2} \mathrm{O}$ (1.5 equiv.) in solvent $(2 \mathrm{~mL})$ at $70{ }^{\circ} \mathrm{C}$ for $2 \mathrm{~h}$; isolated yield; $\mathrm{b}$. quantitative yield of $\mathbf{1}$ recovered in most cases. c. the deuterium ratio was determined by ${ }^{1} \mathrm{H}$ NMR; d: without Mg. 
Table S2. Further Reaction Condition Screening ${ }^{\mathrm{a}}$.<smiles>O=C(c1ccccc1)c1ccccc1</smiles>

$1,1 \mathrm{mmol}$

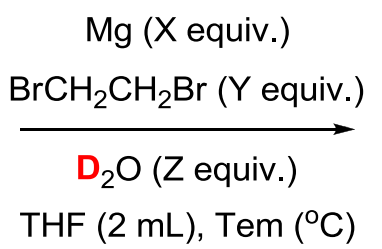<smiles>[2H]C(O)(c1ccccc1)c1ccccc1</smiles>

\begin{tabular}{llllll}
\hline Entry & $\mathrm{Mg}(\mathrm{X}$ equiv. $)$ & Additive (Y equiv. $)$ & $\mathrm{D}_{2} \mathrm{O}(\mathrm{Z}$ equiv. $)$ & $\mathrm{Tem}\left({ }^{\circ} \mathrm{C}\right)$ & Yield \\
\hline 1 & 5.0 & 2.0 & 1.5 & r.t & $56 \%$ \\
2 & 5.0 & 2.0 & 1.5 & 50 & $59 \%$ \\
3 & 4.0 & 2.0 & 1.5 & 70 & $65 \%$ \\
4 & 3.0 & 2.0 & 1.5 & 70 & $40 \%$ \\
5 & 5.0 & 1.0 & 1.5 & 70 & $42 \%$ \\
6 & 5.0 & 3.0 & 1.5 & 70 & $70 \%$ \\
7 & 5.0 & 2.0 & 1.0 & 70 & $61 \%$ \\
8 & 5.0 & 2.0 & 2.0 & 70 & $68 \%$ \\
9 & 5.0 & 2.0 & -- & 70 & $29 \%(0 \% \mathrm{D})$
\end{tabular}

a: Conditions: freshly peeled Mg scraps; isolated yield; the reaction for 2 hours; the deuterium ratio was determined by ${ }^{1} \mathrm{H}$ NMR. 


\section{General procedure for Table S1 and S2:}<smiles>O=C(c1ccccc1)c1ccccc1</smiles>

$1,1 \mathrm{mmol}$

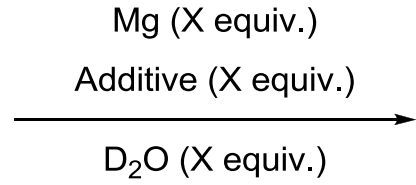

Solvent $(2 \mathrm{~mL})$, Tem $\left({ }^{\circ} \mathrm{C}\right)$<smiles>[2H]C(O)(c1ccccc1)c1ccccc1</smiles>

$1 \mathrm{a}$

To a Schlenk tube with a stirring bar was added Freshly Peeled Mg. The reaction tube was dried by heating under vacuum and refilled with nitrogen. This process was repeated for three times. Solvent $(1 \mathrm{~mL})$ and additive were added into the reaction and the mixture was stirred at $70{ }^{\circ} \mathrm{C}$ in oil bath. Then, benzophenone and $\mathrm{D}_{2} \mathrm{O}$ was dissolved in $1 \mathrm{~mL}$ of solvent and the solution was added into the reaction by syringe and the mixture was stirred at $70{ }^{\circ} \mathrm{C}$ for $2 \mathrm{~h}$ in oil bath. The reaction was cooled to room temperature, followed with quenching and hydrolysis with $10 \mathrm{~mL}$ of saturated aqueous ammonium chloride. After filtration and workup with dichloromethane/water, the organic solution was dried with anhydrous $\mathrm{MgSO}_{4}$ and concentrated under reduced pressure and the residue was purified by column chromatography on silica gel $(\mathrm{PE} / \mathrm{EA}=20 / 1-5 / 1)$ to give the product as a white solid.

General procedure for Figure 3:

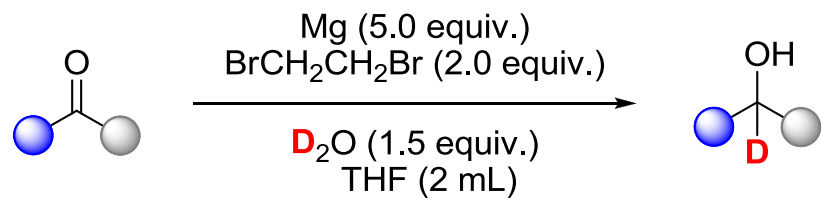

To a Schlenk tube with a stirring bar was added $120 \mathrm{mg}$ (5.0 mmol, 5.0 equiv.) of freshly Peeled Mg. The reaction tube was dried by heating under vacuum and refilled with nitrogen. This process was repeated for three times. Followed by the addition of $1 \mathrm{~mL}$ of THF, the mixture was warmed up to $70{ }^{\circ} \mathrm{C}$ in oil bath. Then, 1,2-dibromoethane (200 uL, $2.0 \mathrm{mmol}, 2.0$ equiv.), a THF solution (1 mL) of ketone (1.0 mmol, 1.0 equiv.) and $\mathrm{D}_{2} \mathrm{O}(30 \mathrm{mg}, 1.5 \mathrm{mmol}, 1.5$ equiv.) were added by syringe sequentially. The mixture was stirred at $70{ }^{\circ} \mathrm{C}$ for $2 \mathrm{~h}$ in oil bath. After completion as detected by TLC, the solution was cooled down to room temperature, and quenched with $10 \mathrm{~mL}$ of saturated aqueous ammonium chloride. After filtration and workup with dichloromethane/water, the organic solution was dried with anhydrous $\mathrm{MgSO}_{4}$ and concentrated under reduced pressure. The residue was purified by column chromatography on silica gel (PE/EA) to give the product. 


\section{The procedure for synthesis of $16 \mathrm{a}$ in $5 \mathbf{~ m m o l ~ s c a l e : ~}$}<smiles>Cc1ccccc1C(=O)c1ccccc1</smiles>

16, $5 \mathrm{mmol}$

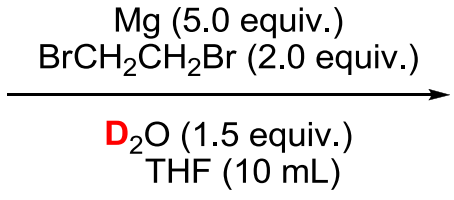

THF $(10 \mathrm{~mL})$<smiles>Cc1ccccc1C(O)(c1ccccc1)c1ccccc1</smiles>

$16 a$

To a Schlenk tube with a stirring bar was added $600 \mathrm{mg}$ ( $25.0 \mathrm{mmol}, 5.0$ equiv.) of freshly Peeled Mg. The reaction tube was dried by heating under vacuum and refilled with nitrogen. This process was repeated for three times. Followed by the addition of $8 \mathrm{~mL}$ of THF, the mixture was warmed up to $70{ }^{\circ} \mathrm{C}$ in oil bath. Then, 1,2-dibromoethane (1000 uL, $10.0 \mathrm{mmol}, 2.0$ equiv.), a THF solution ( $3 \mathrm{~mL}$ ) of ketone (980 mg, $5.0 \mathrm{mmol}, 1.0$ equiv.) and $\mathrm{D}_{2} \mathrm{O}$ (150 mg, $7.5 \mathrm{mmol}, 1.5$ equiv.) were added by syringe sequentially. The mixture was stirred at $70{ }^{\circ} \mathrm{C}$ for $2 \mathrm{~h}$ in oil bath. After completion as detected by TLC, the solution was cooled down to room temperature, and quenched with $30 \mathrm{~mL}$ of saturated aqueous ammonium chloride. After filtration and workup with dichloromethane/water, the organic solution was dried with anhydrous $\mathrm{MgSO}_{4}$ and concentrated under reduced pressure. The residue was purified by column chromatography on silica gel $(\mathrm{PE} / \mathrm{EA}=20 / 1-5 / 1)$ to give the product $16 \mathbf{a}(807.2 \mathrm{mg}, 81 \%$ yield, > 98\% D). 


\section{Characterization data for products}

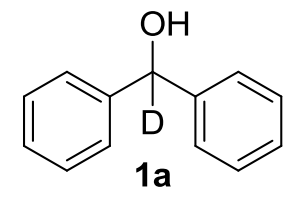

The reaction was performed following the general procedure for Figure 3 and the product was purified by column chromatography on silica gel (PE/EA $=20 / 1-5 / 1)(\mathbf{1 a}$, $154.4 \mathrm{mg}, 83 \%$ yield, > 98\% D, white solid). ${ }^{1} \mathrm{H} \mathrm{NMR}\left(600 \mathrm{MHz}, \mathrm{CDCl}_{3}\right) \delta 7.40-$ $7.32(\mathrm{~m}, 8 \mathrm{H}), 7.31-7.27(\mathrm{~m}, 2 \mathrm{H}), 2.57(\mathrm{br}, 1 \mathrm{H}) .{ }^{13} \mathrm{C} \mathrm{NMR}\left(150 \mathrm{MHz}, \mathrm{CDCl}_{3}\right) \delta$ $143.9,128.6,127.7,126.7,75.9(\mathrm{t}, J=22.6 \mathrm{~Hz})$. HRMS (ESI) calcd for $\left[\mathrm{C}_{13} \mathrm{H}_{10} \mathrm{DO}\right]^{-}$ ([M-H] $\left.]^{\top}\right): 184.0878$, found: $184.0873 . \mathrm{Mp}: 63.5-64.4{ }^{\circ} \mathrm{C} . \mathrm{IR}(\mathrm{KBr}): 3374,3083,3059$, $3025,1494,1448,1189,753,728,699 \mathrm{~cm}^{-1}$.<smiles>OC(O)(c1ccccc1)c1ccc(F)cc1</smiles>

The reaction was performed following the general procedure for Figure 3 and the product was purified by column chromatography on silica gel (PE/EA $=20 / 1-5 / 1)(\mathbf{2 a}$, $142 \mathrm{mg}, 70 \%$ yield, > 99\% D, white solid). ${ }^{1} \mathrm{H}$ NMR $\left(400 \mathrm{MHz}, \mathrm{CDCl}_{3}\right) \delta 7.34-7.22$ $(\mathrm{m}, 7 \mathrm{H}), 6.98(\mathrm{t}, J=8.7 \mathrm{~Hz}, 2 \mathrm{H}), 2.56(\mathrm{br}, 1 \mathrm{H}) .{ }^{13} \mathrm{C} \mathrm{NMR}\left(100 \mathrm{MHz}, \mathrm{CDCl}_{3}\right) \delta 162.2$ $(\mathrm{d}, J=245.7 \mathrm{~Hz}), 143.6,139.5(\mathrm{~d}, J=3.1 \mathrm{~Hz}), 128.6,128.3(\mathrm{~d}, J=8.1 \mathrm{~Hz}), 127.8$, 126.5, $115.3(\mathrm{~d}, J=21.4 \mathrm{~Hz}), 75.2(\mathrm{t}, J=22.2 \mathrm{~Hz}) .{ }^{19} \mathrm{~F}$ NMR $\left(376 \mathrm{MHz}, \mathrm{CDCl}_{3}\right) \delta$ -114.99. HRMS (ESI) calcd for $\left[\mathrm{C}_{13} \mathrm{H}_{9} \mathrm{DFO}\right]^{-}\left([\mathrm{M}-\mathrm{H}]^{-}\right): 202.0784$, found: 202.0780. Mp: 45.8 - $46.3{ }^{\circ} \mathrm{C}$. IR (KBr): 3336, 3060, 3028, 1603, 1508, 1223, 1156, 842, 698 $\mathrm{cm}^{-1}$.<smiles>OC(O)(c1ccccc1)c1cccc(F)c1</smiles>

The reaction was performed following the general procedure for Figure 3 and the product was purified by column chromatography on silica gel (PE/EA $=20 / 1-5 / 1)(\mathbf{3 a}$, $168.5 \mathrm{mg}, 83 \%$ yield, $>97 \% \mathrm{D}$, clean oil). ${ }^{1} \mathrm{H}$ NMR $\left(400 \mathrm{MHz}, \mathrm{CDCl}_{3}\right) \delta 7.34-7.30$ (m, 4H), $7.29-7.21(\mathrm{~m}, 2 \mathrm{H}), 7.12-7.05(\mathrm{~m}, 2 \mathrm{H}), 6.97-6.87(\mathrm{~m}, 1 \mathrm{H}), 2.57(\mathrm{br}, 1 \mathrm{H})$. ${ }^{13} \mathrm{C} \mathrm{NMR}\left(100 \mathrm{MHz}, \mathrm{CDCl}_{3}\right) \delta 162.9(\mathrm{~d}, J=246.0 \mathrm{~Hz}), 146.3(\mathrm{~d}, J=6.8 \mathrm{~Hz}), 143.3$, $130.0(\mathrm{~d}, J=8.2 \mathrm{~Hz}), 128.7,128.0,126.6,122.1(\mathrm{~d}, J=2.8 \mathrm{~Hz}), 114.4(\mathrm{~d}, J=21.2$ $\mathrm{Hz}), 113.4(\mathrm{~d}, J=22.1 \mathrm{~Hz}), 75.3(\mathrm{t}, J=22.2 \mathrm{~Hz}) .{ }^{19} \mathrm{~F} \mathrm{NMR}\left(376 \mathrm{MHz}, \mathrm{CDCl}_{3}\right) \delta$ 
-112.69. HRMS (ESI) calcd for $\left[\mathrm{C}_{13} \mathrm{H}_{9} \mathrm{DFO}\right]^{-}\left([\mathrm{M}-\mathrm{H}]^{-}\right)$: 202.0784 , found: 202.0783. IR (KBr): 3393, 3061, 3028, 1589, 1486, 1448, 1249, 728, $700 \mathrm{~cm}^{-1}$.<smiles>OC(O)(c1ccc(F)cc1)c1ccc(F)cc1</smiles>

The reaction was performed following the general procedure for Figure 3 and the product was purified by column chromatography on silica gel (PE/EA $=20 / 1-5 / 1)(\mathbf{4 a}$, $154.7 \mathrm{mg}, 70 \%$ yield, > 98\% D, clean oil). ${ }^{1} \mathrm{H}$ NMR $\left(400 \mathrm{MHz}, \mathrm{CDCl}_{3}\right) \delta 7.32-7.24$ $(\mathrm{m}, 4 \mathrm{H}), 7.03-6.96(\mathrm{~m}, 4 \mathrm{H}), 2.60(\mathrm{br}, 1 \mathrm{H}) .{ }^{13} \mathrm{C} \mathrm{NMR}\left(100 \mathrm{MHz}, \mathrm{CDCl}_{3}\right) \delta 162.2(\mathrm{~d}$, $J=246.1 \mathrm{~Hz}), 139.4(\mathrm{~d}, J=3.1 \mathrm{~Hz}), 128.2(\mathrm{~d}, J=8.1 \mathrm{~Hz}), 115.4(\mathrm{~d}, J=21.5 \mathrm{~Hz})$, $74.5(\mathrm{t}, J=22.2 \mathrm{~Hz}) .{ }^{19} \mathrm{~F}$ NMR $\left(376 \mathrm{MHz}, \mathrm{CDCl}_{3}\right) \delta-114.67$. HRMS (ESI) calcd for $\left[\mathrm{C}_{13} \mathrm{H}_{8} \mathrm{DF}_{2} \mathrm{O}\right]^{-}\left([\mathrm{M}-\mathrm{H}]^{-}\right): 220.0690$, found: 220.0672. IR (KBr): 3359, 3041, 1603, $1508,1225,1156,1048,834,765 \mathrm{~cm}^{-1}$.<smiles>OC(O)(c1cccc(F)c1)c1cccc(F)c1</smiles>

The reaction was performed following the general procedure for Figure 3 and the product was purified by column chromatography on silica gel (PE/EA $=20 / 1-5 / 1)(\mathbf{5 a}$, $185.7 \mathrm{mg}, 84 \%$ yield, $>98 \% \mathrm{D}$, clean oil). ${ }^{1} \mathrm{H}$ NMR $\left(400 \mathrm{MHz}, \mathrm{CDCl}_{3}\right) \delta 7.28(\mathrm{td}, J=$ 7.9, 6.0 Hz, 2H), $7.14-7.02(\mathrm{~m}, 4 \mathrm{H}), 7.01-6.89(\mathrm{~m}, 2 \mathrm{H}), 2.66(\mathrm{br}, 1 \mathrm{H}) .{ }^{13} \mathrm{C} \mathrm{NMR}$ $\left(100 \mathrm{MHz}, \mathrm{CDCl}_{3}\right) \delta 163.0(\mathrm{~d}, J=246.5 \mathrm{~Hz}), 145.7(\mathrm{~d}, J=6.7 \mathrm{~Hz}), 130.2(\mathrm{~d}, J=8.2$ $\mathrm{Hz}), 122.1(\mathrm{~d}, J=2.9 \mathrm{~Hz}), 114.8(\mathrm{~d}, J=21.2 \mathrm{~Hz}), 113.4(\mathrm{~d}, J=22.2 \mathrm{~Hz}), 74.7$ (t, $J=$ $22.2 \mathrm{~Hz}) .{ }^{19} \mathrm{~F}$ NMR $\left(376 \mathrm{MHz}, \mathrm{CDCl}_{3}\right) \delta$-112.33. HRMS (ESI) calcd for $\left[\mathrm{C}_{13} \mathrm{H}_{8} \mathrm{DF}_{2} \mathrm{O}\right]^{-}\left([\mathrm{M}-\mathrm{H}]^{-}\right): 220.0690$, found: 220.0692. IR $(\mathrm{KBr}): 3368,3068,1613$, $1591,1485,1442,1248,1135,892,787,742 \mathrm{~cm}^{-1}$.<smiles>OC([18OH])(c1ccccc1)c1ccc(F)c(F)c1</smiles>

The reaction was performed following the general procedure for Figure 3 and the product was purified by column chromatography on silica gel $(\mathrm{PE} / \mathrm{EA}=20 / 1-5 / 1)(\mathbf{6 a}$, $90.7 \mathrm{mg}, 41 \%$ yield, > 98\% D, clean oil). ${ }^{1} \mathrm{H}$ NMR (400 MHz, $\left.\mathrm{CDCl}_{3}\right) \delta 7.39-7.15$ $(\mathrm{m}, 6 \mathrm{H}), 7.11-6.97(\mathrm{~m}, 2 \mathrm{H}), 2.41(\mathrm{br}, 1 \mathrm{H}) .{ }^{13} \mathrm{C} \mathrm{NMR}\left(100 \mathrm{MHz}, \mathrm{CDCl}_{3}\right) \delta 151.5(\mathrm{dd}$, $J=67.7,12.7 \mathrm{~Hz}), 149.0(\mathrm{dd}, J=66.7,12.8 \mathrm{~Hz}), 143.1,140.7,128.8,128.1,126.5$, $122.4(\mathrm{dd}, J=6.3,3.6 \mathrm{~Hz}), 116.3(\mathrm{dd}, J=165.4,17.6 \mathrm{~Hz}), 74.8(\mathrm{t}, J=22.2 \mathrm{~Hz}) .{ }^{19} \mathrm{~F}$ NMR (376 MHz, $\left.\mathrm{CDCl}_{3}\right) \delta-137.34(\mathrm{~d}, J=21.2 \mathrm{~Hz}),-139.69(\mathrm{~d}, J=21.2 \mathrm{~Hz})$. HRMS 
(ESI) calcd for $\left[\mathrm{C}_{13} \mathrm{H}_{8} \mathrm{DF}_{2} \mathrm{O}\right]^{-}\left([\mathrm{M}-\mathrm{H}]^{-}\right)$: 220.0690, found: 220.0684. IR (KBr): 3368, $3062,3029,1610,1516,1449,1424,1279,1208,1114,1051,774,725,700 \mathrm{~cm}^{-1}$.

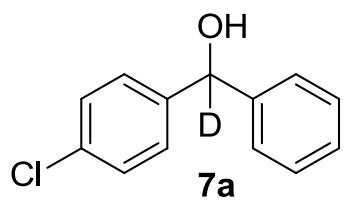

The reaction was performed following the general procedure for Figure 3 and the product was purified by column chromatography on silica gel (PE/EA $=20 / 1-5 / 1)(7 \mathbf{a}$, $164.3 \mathrm{mg}, 75 \%$ yield, $>98 \% \mathrm{D}$, white solid). ${ }^{1} \mathrm{H} \mathrm{NMR}\left(400 \mathrm{MHz}, \mathrm{CDCl}_{3}\right) \delta 7.34-$ $7.20(\mathrm{~m}, 9 \mathrm{H}), 2.56(\mathrm{br}, 1 \mathrm{H}),{ }^{13} \mathrm{C}$ NMR $\left(100 \mathrm{MHz}, \mathrm{CDCl}_{3}\right) \delta 143.4,142.2,133.3$, 128.7, 128.6, 127.9, 127.9, 126.6, $75.2(\mathrm{t}, J=22.2 \mathrm{~Hz}$ ). HRMS (ESI) calcd for $\left[\mathrm{C}_{13} \mathrm{H}_{9} \mathrm{ClDO}^{-}\left([\mathrm{M}-\mathrm{H}]^{-}\right): 218.0488\right.$, found: $218.0485 . \mathrm{Mp}: 55.2-56.2{ }^{\circ} \mathrm{C}$. IR $(\mathrm{KBr})$ : $3377,3060,3027,1600,1489,1448,1399,1189,1089,1048,1014,839,699 \mathrm{~cm}^{-1}$.<smiles>OC(Br)(c1ccccc1)c1cccc(Cl)c1</smiles>

The reaction was performed following the general procedure for Figure 3 and the product was purified by column chromatography on silica gel (PE/EA $=20 / 1-5 / 1)(\mathbf{8 a}$, $184 \mathrm{mg}, 84 \%$ yield, $>98 \% \mathrm{D}$, clean oil). ${ }^{1} \mathrm{H}$ NMR (400 MHz, $\left.\mathrm{CDCl}_{3}\right) \delta 7.36-7.25$ $(\mathrm{m}, 6 \mathrm{H}), 7.23-7.16(\mathrm{~m}, 3 \mathrm{H}), 2.65(\mathrm{br}, 1 \mathrm{H}) .{ }^{13} \mathrm{C} \mathrm{NMR}\left(100 \mathrm{MHz}, \mathrm{CDCl}_{3}\right) \delta 145.7$, 143.2, 134.4, 129.8, 128.7, 128.0, 127.7, 126.6, 124.7, 75.2 (t, $J=22.2 \mathrm{~Hz}$ ). HRMS (ESI) calcd for $\left[\mathrm{C}_{13} \mathrm{H}_{9} \mathrm{ClDO}^{-}\left([\mathrm{M}-\mathrm{H}]^{-}\right): 218.0488\right.$, found: 218.0487 . IR (KBr): 3365, $3061,3027,1595,1573,1474,1448,1421,1189,1048,778,702 \mathrm{~cm}^{-1}$.<smiles>OC(O)(c1ccc(Cl)cc1)c1ccc(Cl)cc1</smiles>

The reaction was performed following the general procedure for Figure 3 and the product was purified by column chromatography on silica gel (PE/EA $=20 / 1-5 / 1)(9 \mathbf{a}$, $200.7 \mathrm{mg}, 79 \%$ yield, > 97\% D, white solid). ${ }^{1} \mathrm{H} \mathrm{NMR}\left(400 \mathrm{MHz}, \mathrm{CDCl}_{3}\right) \delta 7.27$ (d, $J$ $=8.5 \mathrm{~Hz}, 4 \mathrm{H}), 7.22(\mathrm{~d}, J=8.5 \mathrm{~Hz}, 4 \mathrm{H}), 2.70(\mathrm{br}, 1 \mathrm{H}) .{ }^{13} \mathrm{C} \mathrm{NMR}\left(100 \mathrm{MHz}, \mathrm{CDCl}_{3}\right) \delta$ 141.7, 133.6, 128.8, 127.9, $74.5(\mathrm{t}, J=22.2 \mathrm{~Hz})$. HRMS (ESI) calcd for [ $\left.\mathrm{C}_{13} \mathrm{H}_{8} \mathrm{Cl}_{2} \mathrm{DO}\right]^{-}\left([\mathrm{M}-\mathrm{H}]^{-}\right): 252.0099$, found: $252.0097 . \mathrm{Mp}: 85.3-86.2{ }^{\circ} \mathrm{C}$. IR $(\mathrm{KBr})$ : $3351,2921,2850,1486,1400,1200,1089,1058,1014,814,786 \mathrm{~cm}^{-1}$. 
<smiles>OC(O)(c1ccccc1)c1ccc(Cl)c(Cl)c1</smiles>

The reaction was performed following the general procedure for Figure 3 and the product was purified by column chromatography on silica gel $(\mathrm{PE} / \mathrm{EA}=20 / 1-5 / 1)$ (10a, $162.6 \mathrm{mg}, 64 \%$ yield, > 99\% D, clean oil). ${ }^{1} \mathrm{H} \mathrm{NMR}\left(400 \mathrm{MHz}, \mathrm{CDCl}_{3}\right) \delta 7.47$ $(\mathrm{d}, J=2.0 \mathrm{~Hz}, 1 \mathrm{H}), 7.38-7.23(\mathrm{~m}, 7 \mathrm{H}), 7.15(\mathrm{dd}, J=8.3,2.0 \mathrm{~Hz}, 1 \mathrm{H}), 2.57(\mathrm{br}, 1 \mathrm{H})$. ${ }^{13} \mathrm{C} \mathrm{NMR}\left(100 \mathrm{MHz}, \mathrm{CDCl}_{3}\right) \delta 143.8,142.8,132.5,131.4,130.4,128.8,128.4,128.2$, 126.6, 125.8, $74.7(\mathrm{t}, J=22.2 \mathrm{~Hz})$. HRMS (ESI) calcd for $\left[\mathrm{C}_{13} \mathrm{H}_{8} \mathrm{Cl}_{2} \mathrm{DO}\right]^{-}\left([\mathrm{M}-\mathrm{H}]^{-}\right)$: 252.0099, found: 252.0097. IR (KBr): 3360, 3060, 3027, 1468, 1448, 1380, 1189, $1052,1029,700 \mathrm{~cm}^{-1}$.<smiles>N#Cc1ccc(C(O)(O)c2ccccc2)cc1</smiles>

The reaction was performed following the general procedure for Figure 3 and the product was purified by column chromatography on silica gel (PE/EA $=20 / 1-5 / 1)$ (11a, $109.2 \mathrm{mg}$, 52\% yield, > 97\% D, white solid). ${ }^{1} \mathrm{H} \mathrm{NMR}\left(400 \mathrm{MHz}, \mathrm{CDCl}_{3}\right) \delta$ $7.58(\mathrm{~d}, J=8.3 \mathrm{~Hz}, 2 \mathrm{H}), 7.48(\mathrm{~d}, J=8.3 \mathrm{~Hz}, 2 \mathrm{H}), 7.37-7.26(\mathrm{~m}, 5 \mathrm{H}), 2.76$ (br, $1 \mathrm{H})$. ${ }^{13} \mathrm{C} \mathrm{NMR}\left(100 \mathrm{MHz}, \mathrm{CDCl}_{3}\right) \delta 148.9,142.8,132.3,128.9,128.3,127.0,126.7,118.9$, 111.0, $75.2(\mathrm{t}, J=22.2 \mathrm{~Hz})$. HRMS (ESI) calcd for $\left[\mathrm{C}_{14} \mathrm{H}_{10} \mathrm{DNNaO}\right]^{+}\left([\mathrm{M}+\mathrm{Na}]^{+}\right)$: 233.0796, found: 233.0794 . Mp: 68.5 - 69.2 ${ }^{\circ} \mathrm{C}$. IR (KBr): 3420, 3048, 3028, 2231, $1489,1447,1400,1201,1057,847,731,703 \mathrm{~cm}^{-1}$.<smiles>[2H]C(O)(c1ccccc1)c1ccc(C(=O)OC)cc1</smiles>

The reaction was performed following the general procedure for Figure 3 and the product was purified by column chromatography on silica gel $(\mathrm{PE} / \mathrm{EA}=10 / 1-4 / 1)$ (12a, at $50{ }^{\circ} \mathrm{C}$ for 1 hour, $170.3 \mathrm{mg}, 70 \%$ yield, > 96\% D, clean oil). ${ }^{1} \mathrm{H}$ NMR (400 $\left.\mathrm{MHz}, \mathrm{CDCl}_{3}\right) \delta 7.99(\mathrm{~d}, J=8.3 \mathrm{~Hz}, 2 \mathrm{H}), 7.46(\mathrm{~d}, J=8.4 \mathrm{~Hz}, 2 \mathrm{H}), 7.35-7.23(\mathrm{~m}, 5 \mathrm{H})$, $3.89(\mathrm{~s}, 3 \mathrm{H}) .{ }^{13} \mathrm{C} \mathrm{NMR}\left(100 \mathrm{MHz}, \mathrm{CDCl}_{3}\right) \delta 167.0,148.7,143.2,129.8,129.2,128.7$, 128.0, 126.7, 126.3, $75.5(\mathrm{t}, J=22.2 \mathrm{~Hz}$ ), 52.13. HRMS (ESI) calcd for $\left[\mathrm{C}_{15} \mathrm{H}_{13} \mathrm{DNaO}_{3}\right]^{+}\left([\mathrm{M}+\mathrm{Na}]^{+}\right): 266.0898$, found: 266.0899. IR (KBr): 3456, 3207, 2951, $1714,1440,1282,1186,1115,753,702 \mathrm{~cm}^{-1}$. 
<smiles>C=Cc1ccc(C(O)(c2ccccc2)c2ccccc2)cc1</smiles>

The reaction was performed following the general procedure for Figure 3 and the product was purified by column chromatography on silica gel (PE/EA $=20 / 1-5 / 1$ ) (at $70{ }^{\circ} \mathrm{C}$ for 1 hour, 13a, $120.5 \mathrm{mg}, 57 \%$ yield, > 96\% D, white solid). ${ }^{1} \mathrm{H}$ NMR (400 $\left.\mathrm{MHz}, \mathrm{CDCl}_{3}\right) \delta 7.38-7.21(\mathrm{~m}, 9 \mathrm{H}), 6.68(\mathrm{dd}, J=17.6,10.9 \mathrm{~Hz}, 1 \mathrm{H}), 5.72(\mathrm{~d}, J=$ $17.6 \mathrm{~Hz}, 1 \mathrm{H}), 5.22(\mathrm{~d}, J=10.9 \mathrm{~Hz}, 1 \mathrm{H}), 2.37(\mathrm{br}, 1 \mathrm{H}) .{ }^{13} \mathrm{C} \mathrm{NMR}\left(100 \mathrm{MHz}, \mathrm{CDCl}_{3}\right) \delta$ $143.7,143.3,136.9,136.5,128.5,127.6,126.7,126.5,126.3,114.0,75.6(\mathrm{t}, J=22.2$ $\mathrm{Hz})$. HRMS (ESI) calcd for $\left[\mathrm{C}_{15} \mathrm{H}_{13} \mathrm{DNaO}\right]^{+}\left([\mathrm{M}+\mathrm{Na}]^{+}\right): 234.1000$, found: 234.1001. Mp: $66.7-67.2^{\circ} \mathrm{C}$. IR (KBr): 3397, 3057, 3207, 1625, 1495, 1445, 1400, 1183, 1043 , $990,909,844,717 \mathrm{~cm}^{-1}$.<smiles>Cc1ccc(C(O)(c2ccccc2)c2ccccc2)cc1</smiles>

The reaction was performed following the general procedure for Figure 3 and the product was purified by column chromatography on silica gel (PE/EA $=20 / 1-5 / 1)$ (14a, $157.2 \mathrm{mg}, 79 \%$ yield, > 99\% D, white solid). ${ }^{1} \mathrm{H} \mathrm{NMR}\left(400 \mathrm{MHz}, \mathrm{CDCl}_{3}\right) \delta$ $7.37-7.26(\mathrm{~m}, 4 \mathrm{H}), 7.25-7.17(\mathrm{~m}, 3 \mathrm{H}), 7.11(\mathrm{~d}, J=7.9 \mathrm{~Hz}, 2 \mathrm{H}), 2.45$ (br, 1H), 2.30 (s, 3H). $\left.{ }^{13} \mathrm{C} \mathrm{NMR} \mathrm{(100} \mathrm{MHz,} \mathrm{CDCl}_{3}\right) \delta 144.0,141.0,137.3,129.2,128.5,127.5$, 126.6, 126.5, $75.7(\mathrm{t}, J=22.2 \mathrm{~Hz}), 21.2$. HRMS (ESI) calcd for $\left[\mathrm{C}_{14} \mathrm{H}_{13} \mathrm{DNaO}\right]^{+}$ $\left([\mathrm{M}+\mathrm{Na}]^{+}\right): 222.1000$, found: $222.1001 . \mathrm{Mp}: 52.5-53.3{ }^{\circ} \mathrm{C} . \mathrm{IR}(\mathrm{KBr}): 3394,3057$, 3025, 2922, 1513, 1492, 1448, 1190, 1177, 1048, 1019, 727, $698 \mathrm{~cm}^{-1}$.<smiles>Cc1cccc(C(O)(c2ccccc2)c2ccccc2)c1</smiles>

The reaction was performed following the general procedure for Figure 3 and the product was purified by column chromatography on silica gel $(\mathrm{PE} / \mathrm{EA}=20 / 1-5 / 1)$ (15a, $165.3 \mathrm{mg}, 83 \%$ yield, > 98\% D, white solid). ${ }^{1} \mathrm{H}$ NMR (400 $\left.\mathrm{MHz}, \mathrm{CDCl}_{3}\right) \delta$ $7.37-7.28(\mathrm{~m}, 4 \mathrm{H}), 7.26-7.11(\mathrm{~m}, 4 \mathrm{H}), 7.06(\mathrm{~d}, J=7.3 \mathrm{~Hz}, 1 \mathrm{H}), 2.39$ (br, 1H), 2.31 (s, 3H). ${ }^{13} \mathrm{C}$ NMR $\left(100 \mathrm{MHz}, \mathrm{CDCl}_{3}\right) \delta 143.9,143.8,138.2,128.5,128.5,128.4$, 127.6, 127.2, 126.6, 123.7, $75.9(\mathrm{t}, J=22.2 \mathrm{~Hz}), 21$. 6. HRMS (ESI) calcd for $\left[\mathrm{C}_{14} \mathrm{H}_{13} \mathrm{DNaO}\right]^{+}\left([\mathrm{M}+\mathrm{Na}]^{+}\right): 222.1000$, found: $222.1001 . \mathrm{Mp}: 47.7-48.5{ }^{\circ} \mathrm{C}$. IR (KBr): 3393, 3058, 3026, 2920, 1605, 1491, 1448, 1145, 1047, 727, $698 \mathrm{~cm}^{-1}$. 
<smiles>Cc1ccccc1C(O)(O)c1ccccc1</smiles>

The reaction was performed following the general procedure for Figure 3 and the product was purified by column chromatography on silica gel $(\mathrm{PE} / \mathrm{EA}=20 / 1-5 / 1)$ (16a, $167.5 \mathrm{mg}, 84 \%$ yield, $>98 \% \mathrm{D}$, white solid) (The reaction run in $5 \mathrm{mmol}$ scale: $807.2 \mathrm{mg}, 81 \%$ yield, > 98\% D). ${ }^{1} \mathrm{H}$ NMR $\left(400 \mathrm{MHz}, \mathrm{CDCl}_{3}\right) \delta 7.50-7.46(\mathrm{~m}, 1 \mathrm{H})$, $7.31-7.08(\mathrm{~m}, 8 \mathrm{H}), 2.35(\mathrm{br}, 1 \mathrm{H}), 2.21(\mathrm{~s}, 3 \mathrm{H}) .{ }^{13} \mathrm{C} \mathrm{NMR}\left(100 \mathrm{MHz}, \mathrm{CDCl}_{3}\right) \delta 142.8$, $141.4,135.4,130.6,128.5,127.6,127.6,127.2,126.3,126.2,72.9$ (t, $J=22.2 \mathrm{~Hz}$ ), 19.5. HRMS (ESI) calcd for $\left[\mathrm{C}_{14} \mathrm{H}_{13} \mathrm{DNaO}\right]^{+}\left([\mathrm{M}+\mathrm{Na}]^{+}\right): 222.1000$, found: 222.1002 . Mp: 87.5 - $88.2^{\circ} \mathrm{C}$. IR (KBr): 3224, 3062, 3020, 1602, 1488, 1458, 1446, 1182, 1046, $1026,757,735,697 \mathrm{~cm}^{-1}$.<smiles>Cc1ccc(C(O)([18F])c2ccc(C)cc2)cc1</smiles>

The reaction was performed following the general procedure for Figure 3 and the product was purified by column chromatography on silica gel (PE/EA $=20 / 1-5 / 1)$ (17a, $164.1 \mathrm{mg}, 77 \%$ yield, > 99\% D, white solid). ${ }^{1} \mathrm{H}$ NMR $\left(400 \mathrm{MHz}, \mathrm{CDCl}_{3}\right) \delta$ $7.21(\mathrm{~d}, J=8.0 \mathrm{~Hz}, 4 \mathrm{H}), 7.10(\mathrm{~d}, J=8.0 \mathrm{~Hz}, 4 \mathrm{H}), 2.41(\mathrm{br}, 1 \mathrm{H}), 2.30(\mathrm{~s}, 6 \mathrm{H}) .{ }^{13} \mathrm{C}$ NMR $\left(100 \mathrm{MHz}, \mathrm{CDCl}_{3}\right) \delta 141.1,137.2,129.2,126.5,75.5(\mathrm{t}, J=22.2 \mathrm{~Hz}), 21.2$. HRMS (ESI) calcd for $\left[\mathrm{C}_{15} \mathrm{H}_{15} \mathrm{DNaO}\right]^{+}\left([\mathrm{M}+\mathrm{Na}]^{+}\right)$: 236.1156 , found: $236.1156 . \mathrm{Mp}$ : 65.3 - $65.8^{\circ} \mathrm{C}$. IR (KBr): 3437, 3022, 2922, 1646, 1512, 1450, 1179, 1050, 810, 749 $\mathrm{cm}^{-1}$.<smiles>Cc1ccc(C)c(C(O)(c2ccccc2)c2ccccc2)c1</smiles>

The reaction was performed following the general procedure for Figure 3 and the product was purified by column chromatography on silica gel $(\mathrm{PE} / \mathrm{EA}=20 / 1-5 / 1)$ (18a, $164.2 \mathrm{mg}, 77 \%$ yield, > 97\% D, white solid). ${ }^{1} \mathrm{H} \mathrm{NMR}\left(400 \mathrm{MHz}, \mathrm{CDCl}_{3}\right) \delta$ $7.34-7.16(\mathrm{~m}, 6 \mathrm{H}), 7.00(\mathrm{~d}, J=7.8 \mathrm{~Hz}, 1 \mathrm{H}), 6.93(\mathrm{~s}, 1 \mathrm{H}), 2.42(\mathrm{br}, 1 \mathrm{H}), 2.28(\mathrm{~s}, 3 \mathrm{H})$, 2.17 (s, 3H). ${ }^{13} \mathrm{C}$ NMR $\left(100 \mathrm{MHz}, \mathrm{CDCl}_{3}\right) \delta 143.1,138.7,137.2,135.3,131.4,128.5$, $127.5,127.1,126.8,126.5,72.8(\mathrm{t}, J=22.2 \mathrm{~Hz}), 21.1,19.4$. HRMS (ESI) calcd for $\left[\mathrm{C}_{15} \mathrm{H}_{15} \mathrm{DNaO}\right]^{+}\left([\mathrm{M}+\mathrm{Na}]^{+}\right): 236.1156$, found: $236.1154 . \mathrm{Mp}: 47.1-47.9{ }^{\circ} \mathrm{C}$. IR (KBr): 3369, 3024, 2921, 1611, 1496, 1449, 1190, 1039, 829, $701 \mathrm{~cm}^{-1}$. 
<smiles>Cc1ccc(C(O)(O)c2ccccc2)c(C)c1</smiles>

The reaction was performed following the general procedure for Figure 3 and the product was purified by column chromatography on silica gel $(\mathrm{PE} / \mathrm{EA}=20 / 1-5 / 1)$ (19a, $172.7 \mathrm{mg}, 81 \%$ yield, > 97\% D, white solid). ${ }^{1} \mathrm{H} \mathrm{NMR}\left(400 \mathrm{MHz}, \mathrm{CDCl}_{3}\right) \delta$ $7.35-7.28(\mathrm{~m}, 5 \mathrm{H}), 7.26-7.20(\mathrm{~m}, 1 \mathrm{H}), 7.02(\mathrm{~d}, J=7.8 \mathrm{~Hz}, 1 \mathrm{H}), 6.95(\mathrm{~s}, 1 \mathrm{H}), 2.29$ (s, 3H), 2.27 (br, 1H), 2.20 (s, 3H). $\left.{ }^{13} \mathrm{C} \mathrm{NMR} \mathrm{(100} \mathrm{MHz,} \mathrm{CDCl}_{3}\right) \delta$ 143.1, 138.6, 137.2, 135.3, 131.4, 128.5, 127.5, 127.0, 126.8, 126.4, 72.8 (t, $J=22.2 \mathrm{~Hz}), 21.1$, 19.3. HRMS (ESI) calcd for $\left[\mathrm{C}_{15} \mathrm{H}_{15} \mathrm{DNaO}\right]^{+}\left([\mathrm{M}+\mathrm{Na}]^{+}\right): 236.1156$, found: 236.1156 . Mp: 45.9 - $46.3{ }^{\circ} \mathrm{C}$. IR (KBr): 3452, 3022, 2916, 1607, 1492, 1447, 1324, 1184, 1026 , $787,756,696 \mathrm{~cm}^{-1}$.<smiles>Cc1ccc(C(O)(O)c2ccccc2)cc1C</smiles>

The reaction was performed following the general procedure for Figure 3 and the product was purified by column chromatography on silica gel (PE/EA $=20 / 1-5 / 1)$ (20a, $170.6 \mathrm{mg}, 80 \%$ yield, > 98\% D, white solid). ${ }^{1} \mathrm{H} \mathrm{NMR}\left(400 \mathrm{MHz}, \mathrm{CDCl}_{3}\right) \delta$ $7.36-7.17(\mathrm{~m}, 5 \mathrm{H}), 7.12-7.03(\mathrm{~m}, 3 \mathrm{H}), 2.42(\mathrm{br}, 1 \mathrm{H}), 2.21(\mathrm{~s}, 6 \mathrm{H}) .{ }^{13} \mathrm{C}$ NMR $(100$ $\left.\mathrm{MHz}, \mathrm{CDCl}_{3}\right) \delta 144.0,141.5,136.8,136.0,129.8,128.5,127.9,127.4,126.5,124.1$, $75.7(\mathrm{t}, J=21.2 \mathrm{~Hz}), 20.0,19.6$. HRMS $(\mathrm{ESI})$ calcd for $\left[\mathrm{C}_{15} \mathrm{H}_{15} \mathrm{DNaO}\right]^{+}\left([\mathrm{M}+\mathrm{Na}]^{+}\right)$: 236.1156, found: 236.1156 . Mp: $60.2-60.8^{\circ} \mathrm{C}$. IR (KBr): 3361, 3023, 2926, 1498 , $1449,1051,1015,702 \mathrm{~cm}^{-1}$.<smiles>Cc1cc(C)c(C(O)(c2ccccc2)c2ccccc2)c(C)c1</smiles>

21a

The reaction was performed following the general procedure for Figure 3 and the product was purified by column chromatography on silica gel $(\mathrm{PE} / \mathrm{EA}=20 / 1-5 / 1)$ (21a, $195.5 \mathrm{mg}, 86 \%$ yield, > 98\% D, clean oil). ${ }^{1} \mathrm{H} \mathrm{NMR}\left(400 \mathrm{MHz}, \mathrm{CDCl}_{3}\right) \delta 7.31-$ $7.16(\mathrm{~m}, 5 \mathrm{H}), 6.83(\mathrm{~s}, 2 \mathrm{H}), 2.32(\mathrm{br}, 1 \mathrm{H}), 2.26(\mathrm{~s}, 3 \mathrm{H}), 2.20(\mathrm{~s}, 6 \mathrm{H}) .{ }^{13} \mathrm{C}$ NMR $(150$ $\left.\mathrm{MHz}, \mathrm{CDCl}_{3}\right) \delta 143.2,137.5,137.2,136.6,130.2,128.3,126.6,125.7,70.8(\mathrm{t}, J=$ 21.1 Hz), 21.0, 20.7. HRMS (ESI) calcd for $\left[\mathrm{C}_{16} \mathrm{H}_{17} \mathrm{DNaO}\right]^{+}\left([\mathrm{M}+\mathrm{Na}]^{+}\right): 250.1313$, found: 250.1312. IR (KBr): 3435, 3023, 2920, 1608, 1486, 1449, 1184, 1023, 851, $713 \mathrm{~cm}^{-1}$. 
<smiles>[2H]C(O)(c1ccccc1)c1ccc(C(C)(C)C)cc1</smiles>

The reaction was performed following the general procedure for Figure 3 and the product was purified by column chromatography on silica gel $(\mathrm{PE} / \mathrm{EA}=20 / 1-5 / 1)$ (22a, $173.6 \mathrm{mg}, 72 \%$ yield, > 99\% D, white solid). ${ }^{1} \mathrm{H} \mathrm{NMR}\left(400 \mathrm{MHz}, \mathrm{CDCl}_{3}\right) \delta$ $7.38-7.12(\mathrm{~m}, 9 \mathrm{H}), 2.55(\mathrm{br}, 1 \mathrm{H}), 1.28(\mathrm{~s}, 9 \mathrm{H}) .{ }^{13} \mathrm{C} \mathrm{NMR}\left(100 \mathrm{MHz}, \mathrm{CDCl}_{3}\right) \delta 150.5$, 143.9, 140.9, 128.5, 127.5, 126.6, 126.4, 125.5, 75.6 (t, $J=21.2 \mathrm{~Hz}), 34.6,31.5$. HRMS (ESI) calcd for $\left[\mathrm{C}_{17} \mathrm{H}_{19} \mathrm{DNaO}\right]^{+}\left([\mathrm{M}+\mathrm{Na}]^{+}\right): 264.1469$, found: 264.1469 . Mp: 78.2 - $78.7^{\circ} \mathrm{C}$. IR (KBr): 3285, 3057, 3026, 2960, 1512, 1451, 1398, 1048, 839, 743, $698 \mathrm{~cm}^{-1}$.<smiles>OC(O)(c1ccccc1)c1ccc(-c2ccccc2)cc1</smiles>

The reaction was performed following the general procedure for Figure 3 and the product was purified by column chromatography on silica gel $(\mathrm{PE} / \mathrm{EA}=20 / 1-5 / 1)$ (23a, $224.6 \mathrm{mg}, 86 \%$ yield, > 98\% D, white solid). ${ }^{1} \mathrm{H} \mathrm{NMR}\left(400 \mathrm{MHz}, \mathrm{CDCl}_{3}\right) \delta$ $7.56-7.50(\mathrm{~m}, 4 \mathrm{H}), 7.43-7.35(\mathrm{~m}, 6 \mathrm{H}), 7.35-7.28(\mathrm{~m}, 3 \mathrm{H}), 7.28-7.22(\mathrm{~m}, 1 \mathrm{H})$, 2.54 (br, 1H). ${ }^{13} \mathrm{C}$ NMR $\left(100 \mathrm{MHz}, \mathrm{CDCl}_{3}\right) \delta 143.8,142.9,140.8,140.5,128.9,128.6$, 127.7, 127.4, 127.3, 127.2, 127.1, 126.6, 75.6 (t, $J=23.2 \mathrm{~Hz})$. HRMS (ESI) calcd for $\left[\mathrm{C}_{19} \mathrm{H}_{15} \mathrm{DNaO}\right]^{+}\left([\mathrm{M}+\mathrm{Na}]^{+}\right): 284.1156$, found: $284.1154 . \mathrm{Mp}: 93.3-94.0{ }^{\circ} \mathrm{C}$. IR (KBr): 3324, 3056, 3027, 1484, 1448, 1396, 1183, 1046, 999, 844, 755, $698 \mathrm{~cm}^{-1}$.<smiles>COc1ccc(C([14CH3])(O)c2ccccc2)cc1</smiles>

The reaction was performed following the general procedure for Figure 3 and the product was purified by column chromatography on silica gel (PE/EA $=20 / 1-5 / 1$ ) (24a, $176.5 \mathrm{mg}, 82 \%$ yield, > 98\% D, white solid). ${ }^{1} \mathrm{H} \mathrm{NMR}\left(400 \mathrm{MHz}, \mathrm{CDCl}_{3}\right) \delta$ $7.35-7.28(\mathrm{~m}, 4 \mathrm{H}), 7.26-7.20(\mathrm{~m}, 3 \mathrm{H}), 6.83(\mathrm{~d}, J=8.6 \mathrm{~Hz}, 2 \mathrm{H}), 3.74(\mathrm{~s}, 3 \mathrm{H}), 2.51$ (br, 1H). ${ }^{13} \mathrm{C} \mathrm{NMR}\left(100 \mathrm{MHz}, \mathrm{CDCl}_{3}\right) \delta 159.0,144.0,136.2,128.5,128.0,127.5$, 126.5, 113.9, $75.3(\mathrm{t}, J=22.2 \mathrm{~Hz}), 55.31$. HRMS (ESI) calcd for $\left[\mathrm{C}_{14} \mathrm{H}_{13} \mathrm{DNaO}_{2}\right]^{+}$ $\left([\mathrm{M}+\mathrm{Na}]^{+}\right): 238.0949$, found: $238.0949 . \mathrm{Mp}: 61.4-62.2{ }^{\circ} \mathrm{C}$. IR $(\mathrm{KBr}): \mathrm{IR}(\mathrm{KBr})$ : $3434,3026,2939,1610,1509,1451,1300,1245,1175,1034,839,700 \mathrm{~cm}^{-1}$. 
<smiles>COc1ccccc1C(O)(O)c1ccccc1</smiles>

$25 a$

The reaction was performed following the general procedure for Figure 3 and the product was purified by column chromatography on silica gel $(\mathrm{PE} / \mathrm{EA}=20 / 1-5 / 1)$ (25a, $120.5 \mathrm{mg}, 56 \%$ yield, > 97\% D, clean oil). ${ }^{1} \mathrm{H} \mathrm{NMR}\left(400 \mathrm{MHz}, \mathrm{CDCl}_{3}\right) \delta 7.41-$ $7.34(\mathrm{~m}, 2 \mathrm{H}), 7.31(\mathrm{t}, J=7.5 \mathrm{~Hz}, 2 \mathrm{H}), 7.27-7.20(\mathrm{~m}, 3 \mathrm{H}), 6.93(\mathrm{t}, J=7.5 \mathrm{~Hz}, 1 \mathrm{H})$, $6.87(\mathrm{~d}, J=8.1 \mathrm{~Hz}, 1 \mathrm{H}), 3.78(\mathrm{~s}, 3 \mathrm{H}), 3.09$ (br, $1 \mathrm{H}) .{ }^{13} \mathrm{C} \mathrm{NMR}\left(100 \mathrm{MHz}, \mathrm{CDCl}_{3}\right) \delta$ $156.8,143.3,131.9,128.8,128.2,127.9,127.2,126.6,120.8,110.8,71.9(\mathrm{t}, J=22.2$ $\mathrm{Hz})$, 55.4. HRMS (ESI) calcd for $\left[\mathrm{C}_{14} \mathrm{H}_{13} \mathrm{DNaO}_{2}\right]^{+}\left([\mathrm{M}+\mathrm{Na}]^{+}\right): 238.0949$, found:238.0949. IR (KBr): 3392, 3059, 2936, 1594, 1488, 1458, 1239, 1182, 1032 , $749,697 \mathrm{~cm}^{-1}$.<smiles>CCN(CC)CCOc1ccc(C(O)(O)c2ccccc2)cc1</smiles>

26a

The reaction was performed following the general procedure for Figure 3 and the product was purified by column chromatography on silica gel (PE/EA $=5 / 1-1 / 1)$ (at $70{ }^{\circ} \mathrm{C}$ for $10 \mathrm{~min}, \mathbf{2 6 a}, 153.2 \mathrm{mg}, 51 \%$ yield, 93\% D, light yellow oil). ${ }^{1} \mathrm{H}$ NMR (400 $\left.\mathrm{MHz}, \mathrm{CDCl}_{3}\right) \delta 7.39-7.29(\mathrm{~m}, 4 \mathrm{H}), 7.28-7.20(\mathrm{~m}, 3 \mathrm{H}), 6.84(\mathrm{~d}, J=8.6 \mathrm{~Hz}, 2 \mathrm{H})$, $5.77(\mathrm{~s}, 0.07 \mathrm{H}, \mathrm{CHOH}$ of the undeuterated compound), $4.00(\mathrm{t}, J=6.4 \mathrm{~Hz}, 2 \mathrm{H}), 2.83$ $(\mathrm{t}, J=6.3 \mathrm{~Hz}, 2 \mathrm{H}), 2.63-2.57(\mathrm{~m}, 4 \mathrm{H}), 1.05(\mathrm{t}, J=7.1 \mathrm{~Hz}, 6 \mathrm{H}) .{ }^{13} \mathrm{C} \mathrm{NMR}(100 \mathrm{MHz}$, $\left.\mathrm{CDCl}_{3}\right) \delta 158.3,144.1,136.3,128.4,127.9,127.4,126.4,114.4,75.7(\mathrm{CHOH}$ of undeuterated compound), $75.3(\mathrm{t}, J=22.2 \mathrm{~Hz}), 66.4,51.6,47.7,11.7$. HRMS (ESI) calcd for $\left[\mathrm{C}_{19} \mathrm{H}_{25} \mathrm{DNO}_{2}\right]^{+}\left([\mathrm{M}+\mathrm{H}]^{+}\right)$: 301.2021, found: 301.2020. IR (KBr): 3363, $3060,2970,2932,1609,1508,1458,1242,1174,1048,838,700 \mathrm{~cm}^{-1}$.<smiles>CN(C)c1ccc(C(O)(c2ccccc2)c2ccccc2)cc1</smiles>

The reaction was performed following the general procedure for Figure 3 and the product was purified by column chromatography on silica gel (PE/EA $=5 / 1-1 / 1)(\mathbf{2 7 a}$, $109.5 \mathrm{mg}, 48 \%$ yield, $89 \% \mathrm{D}$, light yellow oil). ${ }^{1} \mathrm{H} \mathrm{NMR}\left(400 \mathrm{MHz}, \mathrm{CDCl}_{3}\right) \delta 7.38(\mathrm{~d}$, $J=7.3 \mathrm{~Hz}, 2 \mathrm{H}), 7.32(\mathrm{t}, J=7.5 \mathrm{~Hz}, 2 \mathrm{H}), 7.27-7.15(\mathrm{~m}, 3 \mathrm{H}), 6.68(\mathrm{~d}, J=8.7 \mathrm{~Hz}, 2 \mathrm{H})$, $5.76(\mathrm{~s}, 0.11 \mathrm{H}, \mathrm{CHOH}$ of the undeuterated compound), $2.92(\mathrm{~s}, 6 \mathrm{H}), 2.18(\mathrm{br}, 1 \mathrm{H})$. ${ }^{13} \mathrm{C} \mathrm{NMR}\left(100 \mathrm{MHz}, \mathrm{CDCl}_{3}\right) \delta 150.2,144.2,132.0,128.3,127.8,127.2,126.4,112.5$, 
76.0 ( $\mathrm{CHOH}$ of undeuterated compound), 75.6, ( $\mathrm{t}, J=22.2 \mathrm{~Hz}$ ), 40.7. HRMS (ESI) calcd for $\left[\mathrm{C}_{15} \mathrm{H}_{16} \mathrm{DNaO}\right]^{+}\left([\mathrm{M}+\mathrm{Na}]^{+}\right)$: 251.1265 , found: 251.1261. IR (KBr): 3452, $3025,2928,2889,1610,1519,1447,1349,1161,1094,701 \mathrm{~cm}^{-1}$.<smiles>OC([18OH])(c1ccccc1)c1ccc2c(c1)OCO2</smiles>

The reaction was performed following the general procedure for Figure 3 and the product was purified by column chromatography on silica gel $(\mathrm{PE} / \mathrm{EA}=20 / 1-5 / 1)$ (28a, $114.5 \mathrm{mg}, 63 \%$ yield, > 98\% D, white solid). ${ }^{1} \mathrm{H} \mathrm{NMR}\left(400 \mathrm{MHz}, \mathrm{CDCl}_{3}\right) \delta$ $7.39-7.28(\mathrm{~m}, 4 \mathrm{H}), 7.27-7.22(\mathrm{~m}, 1 \mathrm{H}), 6.82(\mathrm{dt}, J=3.8,2.1 \mathrm{~Hz}, 2 \mathrm{H}), 6.74(\mathrm{~d}, J=$ $8.4 \mathrm{~Hz}, 1 \mathrm{H}), 5.90(\mathrm{~s}, 2 \mathrm{H}), 2.34(\mathrm{br}, 1 \mathrm{H}) .{ }^{13} \mathrm{C} \mathrm{NMR}\left(100 \mathrm{MHz}, \mathrm{CDCl}_{3}\right)$ 147.8, 147.0, 143.7, 138.0, 128.5, 127.6, 126.3, 120.0, 108.1, 107.2, 101.0, 75.6 (t, $J=22.2 \mathrm{~Hz}$ ). HRMS (ESI) calcd for $\left[\mathrm{C}_{14} \mathrm{H}_{11} \mathrm{DNaO}_{3}\right]^{+}\left([\mathrm{M}+\mathrm{Na}]^{+}\right): 252.0741$, found: 252.0740 . IR (KBr): 3398, 3024, 2892, 1492, 1440, 1243, 1039, 936, $702 \mathrm{~cm}^{-1}$.<smiles>COc1ccc(C(O)(c2ccc(F)cc2)c2ccc(F)cc2)cc1</smiles>

The reaction was performed following the general procedure for Figure 3 and the product was purified by column chromatography on silica gel $(\mathrm{PE} / \mathrm{EA}=20 / 1-5 / 1)$ (29a, $149.3 \mathrm{mg}, 64 \%$ yield, > 98\% $\mathrm{D}$, clean oil). ${ }^{1} \mathrm{H} \mathrm{NMR}\left(400 \mathrm{MHz}, \mathrm{CDCl}_{3}\right) \delta 7.31$ $(\mathrm{dd}, J=8.7,5.4 \mathrm{~Hz}, 2 \mathrm{H}), 7.24(\mathrm{~d}, J=8.7 \mathrm{~Hz}, 2 \mathrm{H}), 7.00(\mathrm{t}, J=8.7 \mathrm{~Hz}, 2 \mathrm{H}), 6.86(\mathrm{~d}, J$ $=8.7 \mathrm{~Hz}, 2 \mathrm{H}), 3.78(\mathrm{~s}, 3 \mathrm{H}), 2.34(\mathrm{br}, 1 \mathrm{H}) .{ }^{13} \mathrm{C} \mathrm{NMR}\left(100 \mathrm{MHz}, \mathrm{CDCl}_{3}\right) \delta 162.1(\mathrm{~d}, J$ $=245.5 \mathrm{~Hz}), 159.1,139.7(\mathrm{~d}, J=3.0 \mathrm{~Hz}), 135.9,128.1(\mathrm{~d}, J=8.1 \mathrm{~Hz}), 127.9,115.2(\mathrm{~d}$, $J=21.4 \mathrm{~Hz}), 114.0,74.7(\mathrm{t}, J=23.2 \mathrm{~Hz}), 55.3 .{ }^{19} \mathrm{~F} \mathrm{NMR}\left(376 \mathrm{MHz}, \mathrm{CDCl}_{3}\right) \delta-115.31$. HRMS (ESI) calcd for $\left[\mathrm{C}_{14} \mathrm{H}_{11} \mathrm{DFO}_{2}\right]^{-}\left([\mathrm{M}-\mathrm{H}]^{-}\right)$: 232.0890, found: 232.0872. IR (KBr): $3421,2838,2837,1607,1508,1243,1169,1036,828 \mathrm{~cm}^{-1}$.<smiles>OC(O)(c1ccccc1)c1ccc2ccccc2c1</smiles>

The reaction was performed following the general procedure for Figure 3 and the product was purified by column chromatography on silica gel (PE/EA $=20 / 1-5 / 1)$ (30a, $171.6 \mathrm{mg}, 73 \%$ yield, > 98\% D, white solid). ${ }^{1} \mathrm{H} \mathrm{NMR}\left(400 \mathrm{MHz}, \mathrm{CDCl}_{3}\right) \delta$ $7.80-7.68(\mathrm{~m}, 4 \mathrm{H}), 7.47-7.40(\mathrm{~m}, 2 \mathrm{H}), 7.38-7.33(\mathrm{~m}, 3 \mathrm{H}), 7.32-7.20(\mathrm{~m}, 3 \mathrm{H})$, 
2.65 (br, 1H). ${ }^{13} \mathrm{C}$ NMR (100 MHz, $\left.\mathrm{CDCl}_{3}\right) \delta 143.6,141.1,133.3,132.9,128.6,128.4$, 128.2, 127.8, 127.7, 126.8, 126.3, 126.1, 125.1, 124.9, 75.9 (t, $J=22.2 \mathrm{~Hz}$ ). HRMS (ESI) calcd for $\left[\mathrm{C}_{17} \mathrm{H}_{13} \mathrm{DNaO}\right]^{+}\left([\mathrm{M}+\mathrm{Na}]^{+}\right): 258.1000$, found: 258.1002 . Mp: $77.0-$ $77.5^{\circ} \mathrm{C}$. IR (KBr): 3385, 3055, 3027, 1601, 1498, 1447, 1358, 1167, 1167, 1050, 817 , $748,708 \mathrm{~cm}^{-1}$.

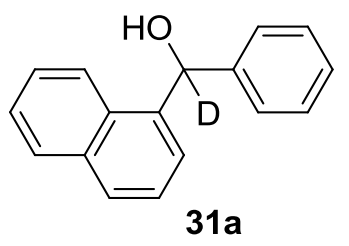

The reaction was performed following the general procedure for Figure 3 and the product was purified by column chromatography on silica gel $(\mathrm{PE} / \mathrm{EA}=20 / 1-5 / 1)$ (31a, $157.6 \mathrm{mg}, 67 \%$ yield, > 99\% $\mathrm{D}$, clean oil). ${ }^{1} \mathrm{H} \mathrm{NMR}\left(400 \mathrm{MHz}, \mathrm{CDCl}_{3}\right) \delta 8.08-$ $7.92(\mathrm{~m}, 1 \mathrm{H}), 7.91-7.75(\mathrm{~m}, 2 \mathrm{H}), 7.60(\mathrm{~d}, J=6.9 \mathrm{~Hz}, 1 \mathrm{H}), 7.52-7.34(\mathrm{~m}, 5 \mathrm{H}), 7.33$ $-7.20(\mathrm{~m}, 3 \mathrm{H}), 2.45$ (br, $1 \mathrm{H}) .{ }^{13} \mathrm{C}$ NMR $\left(100 \mathrm{MHz} \mathrm{CDCl}_{3}\right) \delta 143.1,138.8,134.0$, $130.7,128.8,128.6,128.5,127.7,127.1,126.2,125.7,125.4,124.6,124.0,73.2$ (t, $J$ $=22.2 \mathrm{~Hz})$. HRMS (ESI) calcd for $\left[\mathrm{C}_{17} \mathrm{H}_{13} \mathrm{DNaO}\right]^{+}\left([\mathrm{M}+\mathrm{Na}]^{+}\right): 258.1000$, found: 258.0999. IR (KBr): 3377, 3055, 1595, 1501, 1447, 1168, 1092, 1016, 960, 784, 701 $\mathrm{cm}^{-1}$.<smiles>[2H]C1(O)c2ccccc2-c2ccccc21</smiles>

The reaction was performed following the general procedure for Figure 3 and the product was purified by column chromatography on silica gel $(\mathrm{PE} / \mathrm{EA}=20 / 1-5 / 1)$ (32a, at $40{ }^{\circ} \mathrm{C}, 128.3 \mathrm{mg}, 70 \%$ yield, > 97\% D, white solid). ${ }^{1} \mathrm{H}$ NMR (400 MHz, $\left.\mathrm{CDCl}_{3}\right) \delta 7.60(\mathrm{dd}, J=10.8,7.5 \mathrm{~Hz}, 4 \mathrm{H}), 7.36(\mathrm{t}, J=7.2 \mathrm{~Hz}, 2 \mathrm{H}), 7.29(\mathrm{t}, J=7.3 \mathrm{~Hz}$, 2H), 2.05 (br, 1H). ${ }^{13} \mathrm{C}$ NMR (100 MHz, $\left.\mathrm{CDCl}_{3}\right) \delta 145.6,140.0,129.1,127.8,125.2$, 112.0, 74.8 (t, $J=23.2 \mathrm{~Hz}$ ). HRMS (ESI) calcd for $\left[\mathrm{C}_{13} \mathrm{H}_{8} \mathrm{DO}\right]^{-}\left([\mathrm{M}-\mathrm{H}]^{-}\right)$: 182.0722, found: 182.0715 . Mp: $148.5-149.0^{\circ} \mathrm{C}$. IR (KBr): 3305, 3040, 1445, 1197, 1039, 946, $737 \mathrm{~cm}^{-1}$.<smiles>[2H]C1(O)c2ccccc2-c2cc3ccccc3cc21</smiles>

The reaction was performed following the general procedure for Figure 3 and the product was purified by column chromatography on silica gel $(\mathrm{PE} / \mathrm{EA}=20 / 1-5 / 1)$ 
(33a, $132.9 \mathrm{mg}, 57 \%$ yield, > 98\% D, light yellow solid). ${ }^{1} \mathrm{H}$ NMR (400 MHz, DMSO- $\left.d_{6}\right) \delta 8.26(\mathrm{~s}, 1 \mathrm{H}), 8.09(\mathrm{~s}, 1 \mathrm{H}), 8.01-7.90(\mathrm{~m}, 3 \mathrm{H}), 7.67(\mathrm{~d}, J=7.2 \mathrm{~Hz}, 1 \mathrm{H})$, $7.54-7.35$ (m, 4H), 6.00 (br, 1H). ${ }^{13} \mathrm{C}$ NMR (150 MHz, DMSO- $\left.d_{6}\right) \delta 147.9,145.5$, $139.5,138.7,134.1,133.6,129.3,128.8,128.8,128.6,126.6,126.3,126.0,124.5$, $121.1,118.4,73.3(\mathrm{t}, J=23.2 \mathrm{~Hz})$. HRMS (ESI) calcd for $\left[\mathrm{C}_{17} \mathrm{H}_{11} \mathrm{DNaO}\right]^{+}\left([\mathrm{M}+\mathrm{Na}]^{+}\right)$: 256.0843, found: 256.0840 . Mp: $175.6-176.3{ }^{\circ} \mathrm{C}$. IR (KBr): 3208, 3054, 2925, 1462 , $1231,1187,1115,1042,948,876,752$,<smiles>CC(C)(C)c1ccccc1C1(O)c2ccccc2C1(C)C</smiles>

The reaction was performed following the general procedure for Figure 3 and the product was purified by column chromatography on silica gel $(\mathrm{PE} / \mathrm{EA}=20 / 1-5 / 1)$ (34a, $155.4 \mathrm{mg}, 69 \%$ yield, > 97\% D, white solid). ${ }^{1} \mathrm{H}$ NMR $\left(400 \mathrm{MHz}, \mathrm{CDCl}_{3}\right) \delta$ $7.64(\mathrm{dd}, J=7.5,1.4 \mathrm{~Hz}, 2 \mathrm{H}), 7.55(\mathrm{~d}, J=7.7 \mathrm{~Hz}, 2 \mathrm{H}), 7.37-7.24(\mathrm{~m}, 4 \mathrm{H}), 2.30$ (br, $1 \mathrm{H}), 1.75(\mathrm{~s}, 3 \mathrm{H}), 1.50(\mathrm{~s}, 3 \mathrm{H}) .{ }^{13} \mathrm{C} \mathrm{NMR}\left(100 \mathrm{MHz}, \mathrm{CDCl}_{3}\right) \delta 144.2,137.3,128.1$, 127.1, 126.5, 125.0, $68.3(\mathrm{t}, J=22.2 \mathrm{~Hz}), 38.7,31.4,31.3$. HRMS (ESI) calcd for $\left[\mathrm{C}_{16} \mathrm{H}_{15} \mathrm{DNaO}\right]^{+}\left([\mathrm{M}+\mathrm{Na}]^{+}\right): 248.1156$, found: $248.1154 . \mathrm{Mp}: 130.1-130.7{ }^{\circ} \mathrm{C}$. IR (KBr): 3435, 3062, 2969, 1656, 1589, 1450, 1324, 1284, 1204, 1043, 943, 759, 688 $\mathrm{cm}^{-1}$.

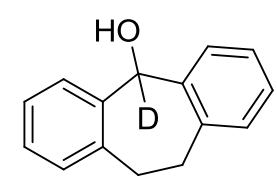

$35 \mathbf{a}$

The reaction was performed following the general procedure for Figure 3 and the product was purified by column chromatography on silica gel (PE/EA $=20 / 1-5 / 1)$ (at $40{ }^{\circ} \mathrm{C}$, 35a, $185.8 \mathrm{mg}, 88 \%$ yield, $>98 \% \mathrm{D}$, white solid). ${ }^{1} \mathrm{H}$ NMR (400 MHz, $\mathrm{CDCl}_{3}$ ) $\delta 7.38(\mathrm{dd}, J=7.1,1.6 \mathrm{~Hz}, 2 \mathrm{H}), 7.22-7.04(\mathrm{~m}, 6 \mathrm{H}), 3.44-3.28(\mathrm{~m}, 2 \mathrm{H}), 3.09-2.97$ $(\mathrm{m}, 2 \mathrm{H}), 2.48(\mathrm{br}, 1 \mathrm{H}) .{ }^{13} \mathrm{C} \mathrm{NMR}\left(100 \mathrm{MHz}, \mathrm{CDCl}_{3}\right) \delta 140.5,139.0,130.2,128.0$, 127.1, 126.2, $76.1(\mathrm{t}, J=23.2 \mathrm{~Hz}), 32.4$. HRMS (ESI) calcd for $\left[\mathrm{C}_{15} \mathrm{H}_{13} \mathrm{DNaO}\right]^{+}$ $\left([\mathrm{M}+\mathrm{Na}]^{+}\right): 234.1000$, found: $234.0999 . \mathrm{Mp}: 84.5-85.2{ }^{\circ} \mathrm{C}$. IR (KBr): 3314, 3062 , 2893, 1480, 1455, 1190, 1040, 980, $747 \mathrm{~cm}^{-1}$.<smiles>[2H]C(O)(CCC)c1ccccc1</smiles>

$36 \mathbf{a}$ 
The reaction was performed following the general procedure for Figure 3 and the product was purified by column chromatography on silica gel $(\mathrm{PE} / \mathrm{EA}=20 / 1-5 / 1)$ (36a, $21.2 \mathrm{mg}, 14 \%$ yield, > 97\% $\mathrm{D}$, clean oil). ${ }^{1} \mathrm{H}$ NMR $\left(600 \mathrm{MHz}, \mathrm{CDCl}_{3}\right) \delta 7.43-$ $7.19(\mathrm{~m}, 5 \mathrm{H}), 1.81-1.77(\mathrm{~m}, 1 \mathrm{H}), 1.71-1.65(\mathrm{~m}, 1 \mathrm{H}), 1.49-1.38(\mathrm{~m}, 1 \mathrm{H}), 1.35-$ $1.27(\mathrm{~m}, 1 \mathrm{H}), 0.93(\mathrm{t}, J=7.4 \mathrm{~Hz}, 3 \mathrm{H}) .{ }^{13} \mathrm{C} \mathrm{NMR}\left(150 \mathrm{MHz}, \mathrm{CDCl}_{3}\right) \delta 144.9,128.5$, 127.6, 126.0, $74.1(\mathrm{t}, J=21.7 \mathrm{~Hz}), 41.2,19.1,14.1$. HRMS $\left(\mathrm{EI}^{+}\right)$calcd for $\left[\mathrm{C}_{10} \mathrm{H}_{13} \mathrm{DO}\right]^{+}\left([\mathrm{M}]^{+}\right):$151.1107, found: 151.1111. IR (KBr): 3370, 2957, 2930, 1698, $1447,760,699 \mathrm{~cm}^{-1}$.<smiles>OC([181In]c1ccccc1)(c1ccccc1)c1ccccn1</smiles>

The reaction was performed following the general procedure for Figure 3 and the product was purified by column chromatography on silica gel $(\mathrm{PE} / \mathrm{EA}=10 / 1-3 / 1)$ (38a, $130.5 \mathrm{mg}, 70 \%$ yield, $87 \% \mathrm{D}$, light yellow solid). ${ }^{1} \mathrm{H} \mathrm{NMR}\left(400 \mathrm{MHz}, \mathrm{CDCl}_{3}\right) \delta$ $8.54(\mathrm{~d}, J=4.6 \mathrm{~Hz}, 1 \mathrm{H}), 7.60(\mathrm{td}, J=7.7,1.4 \mathrm{~Hz}, 1 \mathrm{H}), 7.44-7.21(\mathrm{~m}, 5 \mathrm{H}), 7.20-$ $7.05(\mathrm{~m}, 2 \mathrm{H}), 5.75(\mathrm{~s}, 0.13 \mathrm{H}, \mathrm{CHOH}$ of the undeuterated compound), 5.35 (br, $1 \mathrm{H})$. ${ }^{13} \mathrm{C} \mathrm{NMR}\left(100 \mathrm{MHz}, \mathrm{CDCl}_{3}\right) \delta 160.9,147.9,143.2,136.9,128.6,127.9,127.1,122.5$, 121.4, 75.0 ( $C \mathrm{HOH}$ of the undeuterated compound), $74.6(\mathrm{t}, J=22.2 \mathrm{~Hz}$ ). HRMS $\left[\mathrm{C}_{12} \mathrm{H}_{10} \mathrm{DNNaO}\right]^{+}\left([\mathrm{M}+\mathrm{Na}]^{+}\right): 209.0796$, found: $209.0796 . \mathrm{Mp}: 69.5-70.2{ }^{\circ} \mathrm{C}$. IR (KBr): 3109, 2841, 1588, 1479, 1443, 1204, 1112, 1058, 1001, 754, $694 \mathrm{~cm}^{-1}$.<smiles>OC(c1ccccc1)(c1ccccc1)c1ccncc1</smiles>

The reaction was performed following the general procedure for Figure 3 and the product was purified by column chromatography on silica gel (PE/EA $=10 / 1-3 / 1$ ) (at water bath for 1 hour, 39a, $152.7 \mathrm{mg}, 82 \%$ yield, $89 \% \mathrm{D}$, white solid). ${ }^{1} \mathrm{H}$ NMR (400 $\left.\mathrm{MHz}, \mathrm{CDCl}_{3}\right) \delta 8.29(\mathrm{~d}, J=6.0 \mathrm{~Hz}, 2 \mathrm{H}), 7.39-7.18(\mathrm{~m}, 7 \mathrm{H}), 5.73(\mathrm{~s}, 0.11 \mathrm{H}, \mathrm{CHOH}$ of the undeuterated compound). $\left.{ }^{13} \mathrm{C} \mathrm{NMR} \mathrm{(100} \mathrm{MHz,} \mathrm{CDCl}_{3}\right) \delta 153.5,149.1,143.0$, $128.8,128.1,126.8,121.5,74.7(\mathrm{CHOH}$ of the undeuterated compound), $74.2(\mathrm{t}, J=$ $21.2 \mathrm{~Hz})$. HRMS (ESI) calcd for $\left[\mathrm{C}_{12} \mathrm{H}_{10} \mathrm{DNNaO}\right]^{+}\left([\mathrm{M}+\mathrm{Na}]^{+}\right): 209.0796$, found: 209.0796. Mp: $120.5-121.1{ }^{\circ} \mathrm{C}$. IR (KBr): 3141, 2922, 2816, 1599, 1422, 1197 , $1097,1055,999,756,700 \mathrm{~cm}^{-1}$.<smiles>OC(O)(c1ccncc1)c1ccc(F)cc1</smiles> 
The reaction was performed following the general procedure for Figure 3 and the product was purified by column chromatography on silica gel (PE/EA $=10 / 1-3 / 1$ ) (at water bath for 1 hour, 40a, $124.6 \mathrm{mg}, 61 \%$ yield, $87 \% \mathrm{D}$, white solid). ${ }^{1} \mathrm{H}$ NMR (400 MHz, acetone- $\left.d_{6}\right) \delta 8.47(\mathrm{~d}, J=6.0 \mathrm{~Hz}, 2 \mathrm{H}), 7.46(\mathrm{dd}, J=8.6,5.6 \mathrm{~Hz}, 2 \mathrm{H}), 7.43-$ $7.38(\mathrm{~m}, 2 \mathrm{H}), 7.09(\mathrm{t}, J=8.9 \mathrm{~Hz}, 2 \mathrm{H}), 5.87(\mathrm{~s}, 0.13 \mathrm{H}, \mathrm{CHOH}$ of the undeuterated compound), $5.58(\mathrm{br}, 1 \mathrm{H}) .{ }^{13} \mathrm{C}$ NMR $\left(100 \mathrm{MHz}\right.$, acetone- $\left.d_{6}\right) \delta 162.1(\mathrm{~d}, J=243.7 \mathrm{~Hz})$, $153.8,149.5,140.5,128.5(\mathrm{~d}, J=8.2 \mathrm{~Hz}), 121.2,115.0(\mathrm{~d}, J=21.6 \mathrm{~Hz}) .73 .3(\mathrm{CHOH}$ of the undeuterated compound), $72.9(\mathrm{t}, J=22.2 \mathrm{~Hz}) .{ }^{19} \mathrm{~F}$ NMR $\left(376 \mathrm{MHz}\right.$, acetone- $\left.d_{6}\right)$ $\delta$-116.70. HRMS (ESI) calcd for $\left[\mathrm{C}_{12} \mathrm{H}_{10} \mathrm{DFNO}\right]^{+}\left([\mathrm{M}+\mathrm{H}]^{+}\right)$: 205.0882, found: 205.0883. Mp: $124.2-124.9{ }^{\circ} \mathrm{C}$. IR (KBr): 3120, 2825, 1602, 1504, 1413, 1224 , $1060,1002,825,784,756 \mathrm{~cm}^{-1}$.<smiles>OC(c1ccc(Cl)cc1)(c1ccccn1)c1ccccn1</smiles>

The reaction was performed following the general procedure for Figure 3 and the product was purified by column chromatography on silica gel (PE/EA $=10 / 1-3 / 1$ ) (at water bath for 1 hour, 41a, $171.8 \mathrm{mg}, 78 \%$ yield, $88 \% \mathrm{D}$, clean oil). ${ }^{1} \mathrm{H}$ NMR (400 $\left.\mathrm{MHz}, \mathrm{CDCl}_{3}\right) \delta 8.51(\mathrm{~d}, J=4.5 \mathrm{~Hz}, 1 \mathrm{H}), 7.61(\mathrm{td}, J=7.8,1.6 \mathrm{~Hz}, 1 \mathrm{H}), 7.36-7.22(\mathrm{~m}$, $4 \mathrm{H}), 7.21-7.08(\mathrm{~m}, 2 \mathrm{H}), 5.72(\mathrm{~s}, 0.12 \mathrm{H}, \mathrm{CHOH}$ of the undeuterated compound $), 5.49$ (br, 1H). ${ }^{13} \mathrm{C} \mathrm{NMR}\left(100 \mathrm{MHz}, \mathrm{CDCl}_{3}\right) \delta 160.6,148.0,141.7,137.1,133.6,128.7$, $128.4,122.7,121.2,74.4(\mathrm{CHOH}$ of the undeuterated compound), 74.0 ( $\mathrm{t}, J=22.2$ $\mathrm{Hz})$. HRMS (ESI) calcd for $\left[\mathrm{C}_{12} \mathrm{H}_{9} \mathrm{ClDNNaO}\right]^{+}\left([\mathrm{M}+\mathrm{Na}]^{+}\right): 243.0406$, found: 243.0406. IR (KBr): 3185, 3061, 1592, 1482, 1431, 1397, 1083, 1005, 797, $757 \mathrm{~cm}^{-1}$.<smiles>OC1(O)c2ccc(Cl)cc2CCc2ncccc21</smiles>

The reaction was performed following the general procedure for Figure 3 and the product was purified by column chromatography on silica gel (PE/EA $=10 / 1-3 / 1)$ (at $40{ }^{\circ} \mathrm{C}$ for 2 hours, $42 \mathrm{a}, 216.6 \mathrm{mg}, 88 \%$ yield, $82 \% \mathrm{D}$, light yellow oil). ${ }^{1} \mathrm{H}$ NMR (400 $\left.\mathrm{MHz}, \mathrm{CDCl}_{3}\right) \delta 8.29(\mathrm{~d}, J=4.6 \mathrm{~Hz}, 1 \mathrm{H}), 7.68(\mathrm{~d}, J=8.3 \mathrm{~Hz}, 1 \mathrm{H}), 7.36(\mathrm{~d}, J=7.6 \mathrm{~Hz}$, $1 \mathrm{H}), 7.18(\mathrm{dd}, J=8.3,2.0 \mathrm{~Hz}, 1 \mathrm{H}), 7.15-7.06(\mathrm{~m}, 2 \mathrm{H}), 6.45(\mathrm{br}, 1 \mathrm{H}), 6.20(\mathrm{~s}, 0.18 \mathrm{H}$, $\mathrm{CHOH}$ of the undeuterated compound), $3.49-3.21(\mathrm{~m}, 2 \mathrm{H}), 2.99-2.70(\mathrm{~m}, 2 \mathrm{H}) .{ }^{13} \mathrm{C}$ NMR $\left(100 \mathrm{MHz}, \mathrm{CDCl}_{3}\right) \delta 155.0,144.4,140.9,139.1,138.8,132.6,132.1,128.3$, 126.6, 125.2, 122.9, 68.8 ( $\mathrm{CHOH}$ of the undeuterated compound), 68.4 (t, $J=22.2$ $\mathrm{Hz}), 31.5,30.3$. HRMS (ESI) calcd for $\left[\mathrm{C}_{14} \mathrm{H}_{10} \mathrm{ClDNO}^{-}\left([\mathrm{M}-\mathrm{H}]^{-}\right): 245.0597\right.$, found: 245.0597. IR (KBr): 3305, 3056, 2926, 1666, 1585, 1449, 1422, 1296, 1194, 946, $841,799 \mathrm{~cm}^{-1}$. 
<smiles>O=C(c1ccccc1)C(O)(c1ccccc1)c1ccccc1</smiles>

The reaction was performed following the general procedure for Figure 3 and the product was purified by column chromatography on silica gel (PE/EA $=20 / 1-5 / 1)(\mathrm{Mg}$ 10 equiv., $\mathrm{BrCH}_{2} \mathrm{CH}_{2} \mathrm{Br} 4.0$ equiv., $\mathrm{D}_{2} \mathrm{O} 3.0$ equiv., THF $3 \mathrm{~mL}, \mathbf{4 3 a}, 106.7 \mathrm{mg}, 50 \%$ yield, > 98\% D, white solid). ${ }^{1} \mathrm{H}$ NMR $\left(400 \mathrm{MHz} \mathrm{CDCl}_{3}\right) \delta 7.91(\mathrm{~d}, J=7.4 \mathrm{~Hz}, 2 \mathrm{H})$, $7.51(\mathrm{t}, J=7.4 \mathrm{~Hz}, 1 \mathrm{H}), 7.42-7.23(\mathrm{~m}, 7 \mathrm{H}), 4.57$ (br, $1 \mathrm{H}) .{ }^{13} \mathrm{C}$ NMR $(100 \mathrm{MHz}$, $\left.\mathrm{CDCl}_{3}\right) \delta 199.0,139.0,134.0,133.5,129.2,128.7,128.7,127.8,75.8(\mathrm{t}, J=23.2 \mathrm{~Hz})$. HRMS (ESI) calcd for $\left[\mathrm{C}_{14} \mathrm{H}_{11} \mathrm{DNaO}_{2}\right]^{+}\left([\mathrm{M}+\mathrm{Na}]^{+}\right)$: 236.0792 , found: $236.0792 . \mathrm{Mp}$ : 127.4 - $127.9^{\circ} \mathrm{C}$. IR (KBr): 3378, 3057, 3024, 1675, 1587, 1444, 1331, 1259, 1113 , $753,696 \mathrm{~cm}^{-1}$.<smiles>[2H]C(O)(c1ccccc1)c1ccc(C(=O)c2ccccc2)cc1</smiles>

The reaction was performed following the general procedure for Figure 3 and the product was purified by column chromatography on silica gel (PE/EA $=20 / 1-5 / 1)(\mathrm{Mg}$ 10 eq., $\mathrm{BrCH}_{2} \mathrm{CH}_{2} \mathrm{Br} 4.0$ equiv., $\mathrm{D}_{2} \mathrm{O} 3.0$ equiv., THF $3 \mathrm{~mL}, 44 \mathrm{a}, 161.8 \mathrm{mg}, 56 \%$ yield, $72 \% \mathrm{D}$, light yellow oil). ${ }^{1} \mathrm{H} \mathrm{NMR}\left(400 \mathrm{MHz}, \mathrm{CDCl}_{3}\right) \delta 7.89-7.70(\mathrm{~m}, 4 \mathrm{H}), 7.58(\mathrm{t}$, $J=7.4 \mathrm{~Hz}, 1 \mathrm{H}), 7.52-7.43(\mathrm{~m}, 4 \mathrm{H}), 7.41-7.32(\mathrm{~m}, 4 \mathrm{H}), 7.32-7.26(\mathrm{~m}, 1 \mathrm{H}), 5.90$ (s, $0.28 \mathrm{H}, \mathrm{CHOH}$ of the undeuterated compound), 2.63 (br, $1 \mathrm{H}) .{ }^{13} \mathrm{C} \mathrm{NMR}(100 \mathrm{MHz}$, $\left.\mathrm{CDCl}_{3}\right) \delta 196.5,148.3(\mathrm{CHOH}$ of the undeuterated compound), 148.3, $143.3(\mathrm{CHOH}$ of the undeuterated compound), 143.2, 137.6, 136.6, 132.5, 130.4, 130.1, 128.8, 128.3, 128.0, 126.7, 126.3, 76.0 ( $\mathrm{CHOH}$ of the undeuterated compound), $75.5(\mathrm{t}, J=21.2$ $\mathrm{Hz})$. HRMS (ESI) calcd for $\left[\mathrm{C}_{20} \mathrm{H}_{15} \mathrm{DNaO}_{2}\right]^{+}\left([\mathrm{M}+\mathrm{Na}]^{+}\right): 312.1105$, found: 312.1103 . IR (KBr): 3443, 3058, 3029, 1651, 1602, 1312, 1278, 1184, 1051, 928, 746, $702 \mathrm{~cm}^{-1}$. 


\section{Synthesis of deuterium-labelled drugs and pharmaceutical}

\section{precursors}

\section{a) Reductive deuteration of Fenofibrate}<smiles>[R5]C(C)(OC(=O)C(C)(C)Oc1ccc(C([2H])(O)c2ccc(Cl)cc2)cc1)C(C)C</smiles>

The reaction was performed following the general procedure for Figure 3 and the product was purified by column chromatography on silica gel (PE/EA $=10 / 1-3 / 1)(1$ mmol of 45, 1.0 equiv. of $\mathrm{BrCH}_{2} \mathrm{CH}_{2} \mathrm{Br}, 1.0$ equiv. of $\mathrm{LiBF}_{4}$, at $40{ }^{\circ} \mathrm{C}$ for $20 \mathrm{~min}$ ). (45a, $141.8 \mathrm{mg}, 39 \%$ yield, > 97\% D, clean oil). ${ }^{1} \mathrm{H} \mathrm{NMR}\left(400 \mathrm{MHz}, \mathrm{CDCl}_{3}\right) \delta 7.30-$ $7.28(\mathrm{~m}, 4 \mathrm{H}), 7.18(\mathrm{~d}, J=8.8 \mathrm{~Hz}, 2 \mathrm{H}), 6.80(\mathrm{~d}, J=8.7 \mathrm{~Hz}, 2 \mathrm{H}), 5.06(\mathrm{p}, J=6.3 \mathrm{~Hz}$, $1 \mathrm{H}), 2.26(\mathrm{br}, 1 \mathrm{H}), 1.57(\mathrm{~s}, 6 \mathrm{H}), 1.20(\mathrm{~d}, J=6.3 \mathrm{~Hz}, 6 \mathrm{H}) .{ }^{13} \mathrm{C} \mathrm{NMR}\left(150 \mathrm{MHz}, \mathrm{CDCl}_{3}\right)$ $\delta 173.8,155.3,142.3,136.9,133.2,128.6,127.9,127.6,119.0,79.2,74.8(\mathrm{t}, J=21.1$ $\mathrm{Hz}), 69.1,25.4,21.6$. HRMS (ESI) calcd for $\left[\mathrm{C}_{20} \mathrm{H}_{22} \mathrm{ClDNaO}_{4}\right]^{+}\left([\mathrm{M}+\mathrm{Na}]^{+}\right): 386.1240$, found: 386.1243. IR (KBr): 3497, 2984, 2933, 1727, 1606, 1503, 1380, 1286, 1235 , $1176,1152,1100,832 \mathrm{~cm}^{-1}$.

\section{b) Synthesis of deuterated Diphenhydramine}<smiles>[2H]C(O)(c1ccccc1)c1ccccc1</smiles>

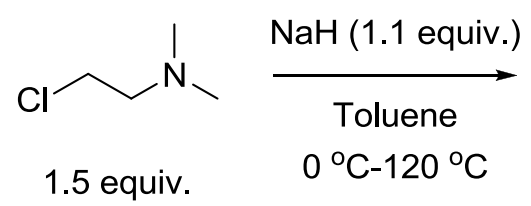<smiles>[2H]C(OCCN(C)C)(c1ccccc1)c1ccccc1</smiles>

To a toluene $(1.5 \mathrm{~mL})$ solution of $\alpha$-deuterated benzhydrol (1a, $92.5 \mathrm{mg}, 0.5 \mathrm{mmol}$, 1.0 equiv.) was added $\mathrm{NaH}(22 \mathrm{mg}, 0.55 \mathrm{mmol}, 1.1$ equiv., $60 \%$ dispersion in nimeral oil) at $0{ }^{\circ} \mathrm{C}$ under nitrogen atmosphere. The solution was stirred at $90{ }^{\circ} \mathrm{C}$ for $30 \mathrm{~min}$. The toluene ( $1 \mathrm{~mL}$ ) solution of $N, N$-dimethylaminoethyl chloride (1.0 equiv.) was added to the mixture and the reaction was stirred under reflux for $5 \mathrm{~h}$. The solution was cooled to room temperature and workup with dichloromethane/water, the organic solution was treated with $2 \mathrm{~N} \mathrm{HCl}$. The acid phase was alkalized and extracted with DCM. The organic solution was dried with anhydrous $\mathrm{MgSO}_{4}$ and concentrated under reduced pressure. The product $\mathbf{4 6 a}$ was obtained as clean oil $(116.2 \mathrm{mg}, 91 \%$ yield, > $98 \%$ D). ${ }^{1} \mathrm{H}$ NMR $\left(400 \mathrm{MHz}, \mathrm{CDCl}_{3}\right) \delta 7.38-7.26(\mathrm{~m}, 8 \mathrm{H}), 7.23$ (dd, $J=11.2,4.2$ $\mathrm{Hz}, 2 \mathrm{H}), 3.56(\mathrm{t}, J=6.0 \mathrm{~Hz}, 2 \mathrm{H}), 2.60(\mathrm{t}, J=6.0 \mathrm{~Hz}, 2 \mathrm{H}), 2.26(\mathrm{~s}, 6 \mathrm{H}) .{ }^{13} \mathrm{C} \mathrm{NMR}$ $\left(100 \mathrm{MHz}, \mathrm{CDCl}_{3}\right) \delta 142.2,128.4,127.4,127.0,83.6(\mathrm{t}, J=22.2 \mathrm{~Hz}), 67.5,59.0,46.0$. 
HRMS (ESI) calcd for $\left[\mathrm{C}_{17} \mathrm{H}_{21} \mathrm{DNO}\right]^{+}\left([\mathrm{M}+\mathrm{H}]^{+}\right)$: 257.1759 , found: 257.1760 . IR (KBr): 3023, 2938, 1484, 1453, 1197, 1106, 1033, 746, $702 \mathrm{~cm}^{-1}$.

\section{c) Synthesis of deuterated Buclizine}

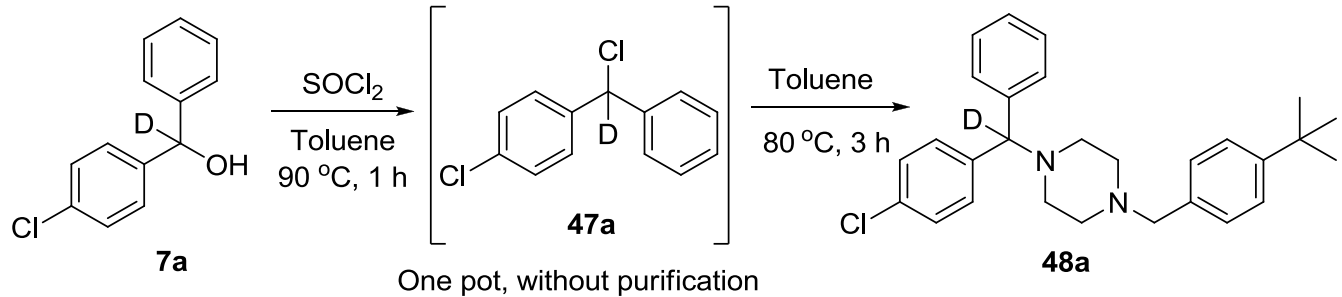

To a toluene $(1 \mathrm{~mL})$ solution of $\alpha$-deuterated-4-chlorobenzhydrol (7a, $109.5 \mathrm{mg}, 0.5$ mmol, 1.0 equiv.) was added thionyl chloride ( $76.7 \mathrm{mg}, 0.65 \mathrm{mmol}, 1.3$ equiv.) at r.t. The reaction mixture was heated to $90{ }^{\circ} \mathrm{C}$ and stirred for $1 \mathrm{~h}$. The solution was cooled to room temperature and evaporated to dryness. Toluene $(1 \mathrm{~mL})$ was added to the residue and the solvent was removed again in vacuo. The residue was dissolved in toluene $(1 \mathrm{~mL})$, and a toluene $(1 \mathrm{~mL})$ solution of 1-(4-tert-butylbenzyl)piperazine (255 mg, $1.1 \mathrm{mmol} 2.2$ equiv.) was added dropwise at $80{ }^{\circ} \mathrm{C}$. The reaction mixture was stirred at $80{ }^{\circ} \mathrm{C}$ for $3 \mathrm{~h}$. The solution was cooled to room temperature. After workup with dichloromethane/water, the organic solution was dried with anhydrous $\mathrm{MgSO}_{4}$ and concentrated under reduced pressure and the residue was purified by column chromatography on silica gel $(\mathrm{PE} / \mathrm{EA}=10 / 1-2 / 1)$ to give the 48a (110 mg, $51 \%$ yield, $>98 \% \mathrm{D})$ as light yellow oil. ${ }^{1} \mathrm{H}$ NMR $\left(600 \mathrm{MHz}, \mathrm{CDCl}_{3}\right) \delta 7.35-7.28$ $(\mathrm{m}, 6 \mathrm{H}), 7.26-7.15(\mathrm{~m}, 7 \mathrm{H}), 3.47(\mathrm{~s}, 2 \mathrm{H}), 2.64-2.17(\mathrm{~m}, 8 \mathrm{H}), 1.29(\mathrm{~s}, 9 \mathrm{H}) .{ }^{13} \mathrm{C}$ NMR $\left(150 \mathrm{MHz}, \mathrm{CDCl}_{3}\right) \delta 150.0,142.2,141.4,134.9,132.5,129.3,129.1,128.7$, 128.6, 128.0, 127.2, 125.1, 62.8, 53.4, 51.9, 34.5, 31.5. HRMS (ESI) calcd for $\left[\mathrm{C}_{28} \mathrm{H}_{33} \mathrm{DClN}_{2}\right]^{+}\left([\mathrm{M}+\mathrm{H}]^{+}\right): 434.2468$, found: 434.2468. IR (KBr): 3056, 3025, 2959, $2807,1691,1514,1484,1458,1277,1141,1095,1009,847,751,704 \mathrm{~cm}^{-1}$.

\section{d) Synthesis of deuterated precursor of deuterated Modafinil and Adrafinil}<smiles>[2H]C(O)(c1ccccc1)c1ccccc1</smiles>

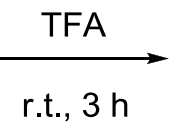<smiles>[2H]C(SCC(=O)O)(C(=O)O)c1ccccc1</smiles>

A mixture of $\alpha$-deuterated benzhydrol (1a, $92.5 \mathrm{mg}, 0.5 \mathrm{mmol}, 1.0$ equiv.) and thioglycolic acid (46 mg, $0.5 \mathrm{mmol}, 1.0$ equiv.) in trifluoroacetic acid (0.6 mL) was stirred at room temperature for $3 \mathrm{~h}$. The solvent was removed under reduced pressure to afford a crude solid. $\mathrm{H}_{2} \mathrm{O}(10 \mathrm{~mL})$ was added and the resulting precipitate collected by filtration. The solid was washed with hexanes $(10 \mathrm{~mL})$ and dried to afford 49a (127.5 mg, 99\% yield, > 98\% D) as a white solid. ${ }^{1} \mathrm{H}$ NMR (400 $\left.\mathrm{MHz}, \mathrm{CDCl}_{3}\right) \delta$ 
10.57 (br, 1H), 7.44 (d, $J=7.4 \mathrm{~Hz}, 4 \mathrm{H}), 7.31$ (t, $J=7.5 \mathrm{~Hz}, 4 \mathrm{H}), 7.22(\mathrm{~d}, J=7.3 \mathrm{~Hz}$, $2 \mathrm{H}), 3.08(\mathrm{~s}, 2 \mathrm{H}) .{ }^{13} \mathrm{C} \mathrm{NMR}\left(100 \mathrm{MHz}, \mathrm{CDCl}_{3}\right) \delta 177.1,140.0,128.8,128.5,127.7$, $53.8(\mathrm{t}, J=22.2 \mathrm{~Hz}), 33.5$. HRMS (ESI) calcd for $\left[\mathrm{C}_{15} \mathrm{H}_{12} \mathrm{DO}_{2} \mathrm{~S}\right]^{-}\left([\mathrm{M}-\mathrm{H}]^{-}\right): 258.0705$, found: 258.0705 . Mp: $119.1-120.0{ }^{\circ} \mathrm{C}$. IR (KBr): 3023, 2917, 2665, 2570, 1699 , $1489,1426,1305,1136,938,746,694 \mathrm{~cm}^{-1}$. 


\section{NMR spectra}

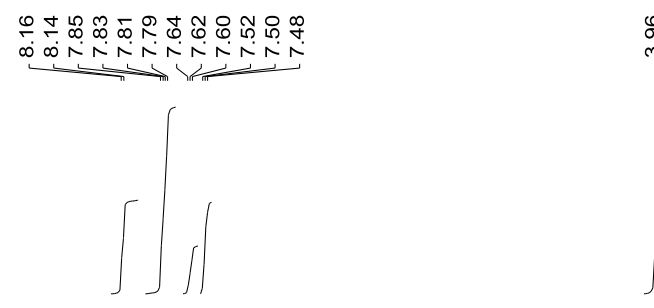<smiles>COC(=O)c1ccc(C(=O)c2ccccc2)cc1</smiles>

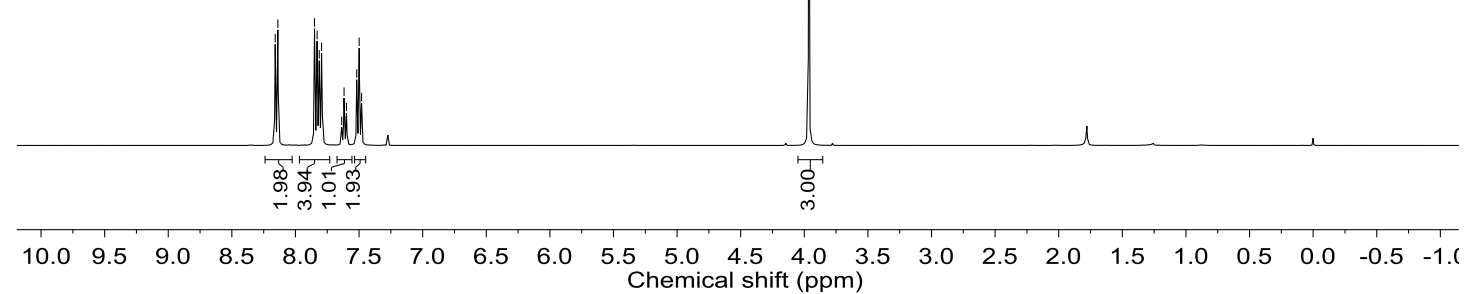

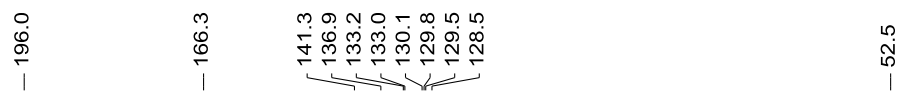<smiles>COC(=O)c1ccc(C(=O)c2ccccc2)cc1</smiles>

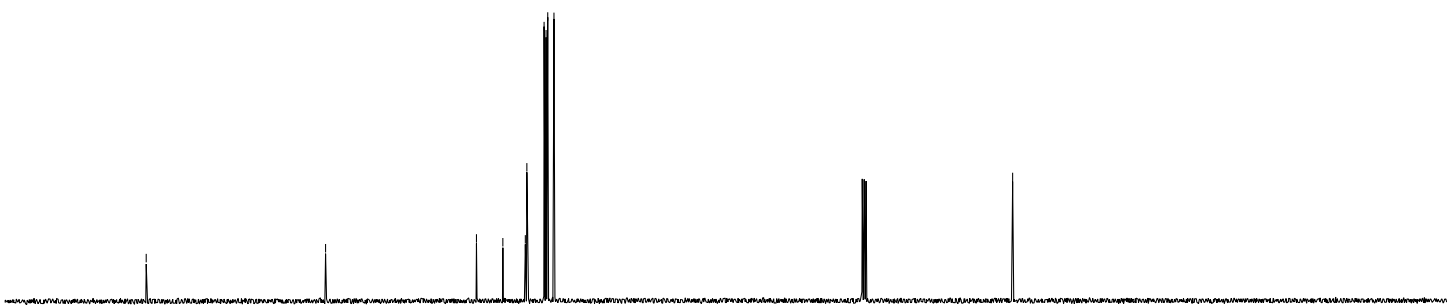

$\begin{array}{llllllllllllllllllllllll}210 & 200 & 190 & 180 & 170 & 160 & 150 & 140 & 130 & 120 & 110 & 100 & 90 & 80 & 70 & 60 & 50 & 40 & 30 & 20 & 10 & 0 & -10\end{array}$ Chemical shift (ppm) 


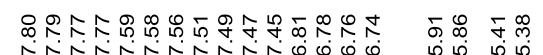

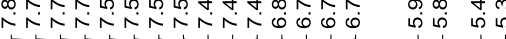

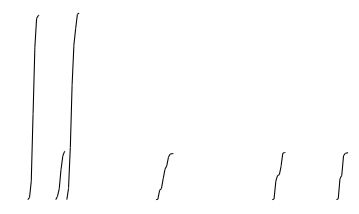

(N)

13

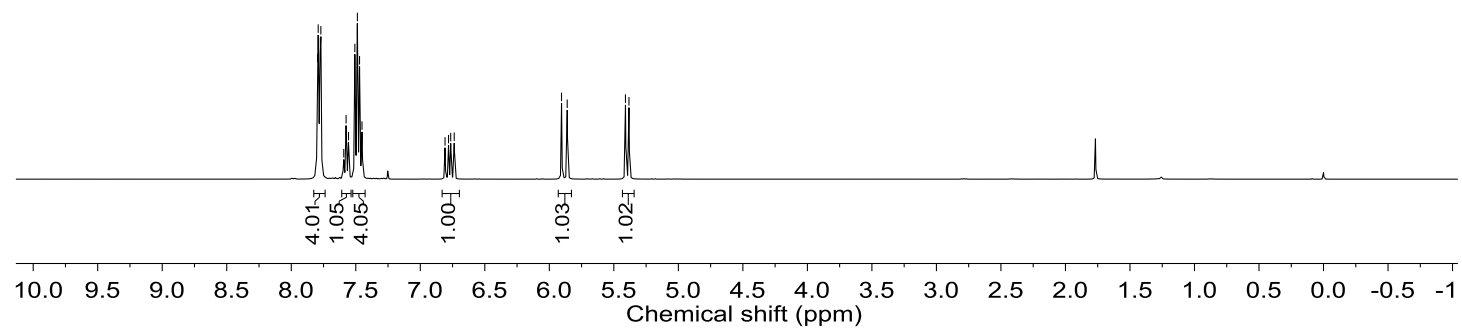

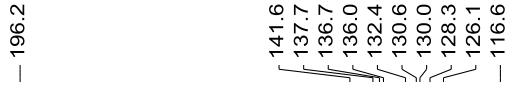<smiles>C=Cc1ccc(C(=O)c2ccccc2)cc1</smiles>

13

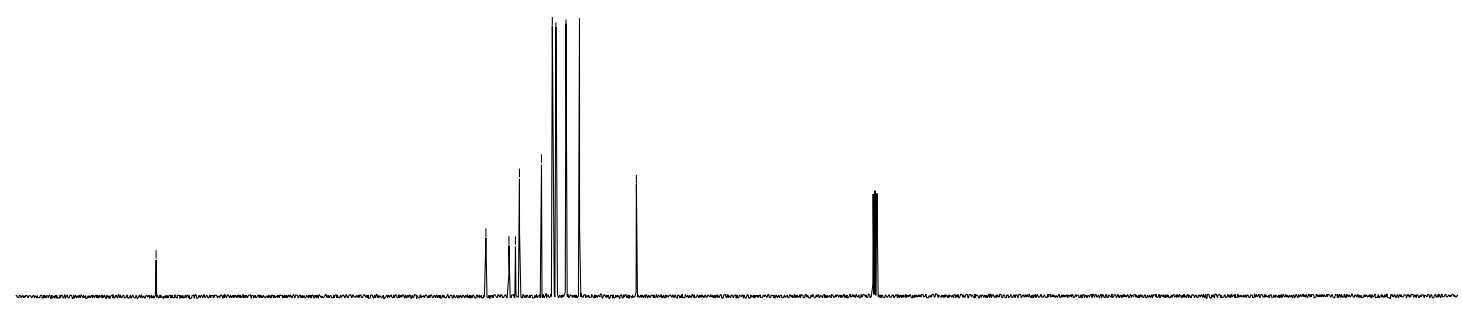

$\begin{array}{lllllllllllllllllllllll}210 & 200 & 190 & 180 & 170 & 160 & 150 & 140 & 130 & 120 & 110 & 100 & 90 & 80 & 70 & 60 & 50 & 40 & 30 & 20 & 10 & 0 & -10\end{array}$ 


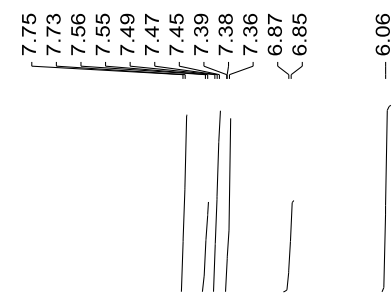<smiles>O=C(c1ccccc1)c1ccc2c(c1)OCO2</smiles>

28

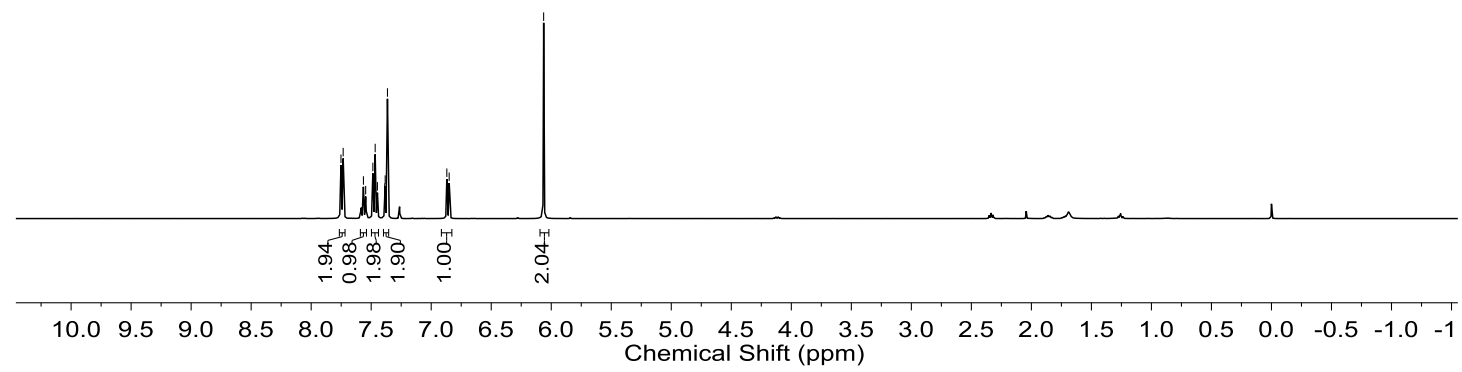

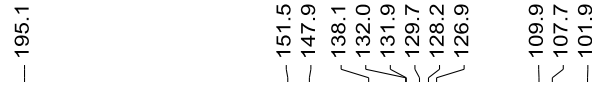<smiles>O=C(c1ccccc1)c1ccc2c(c1)OCO2</smiles>

28

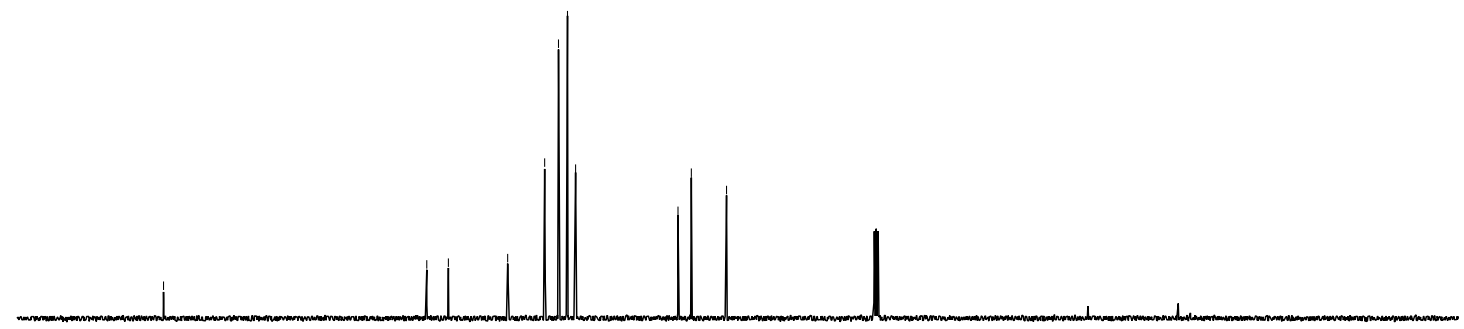

$\begin{array}{llllllllllllllllllllllll}210 & 200 & 190 & 180 & 170 & 160 & 150 & 140 & 130 & 120 & 110 & 100 & 90 & 80 & 70 & 60 & 50 & 40 & 30 & 20 & 10 & 0 & -10\end{array}$ 
<smiles>[2H]C(O)(c1ccccc1)c1ccccc1</smiles>

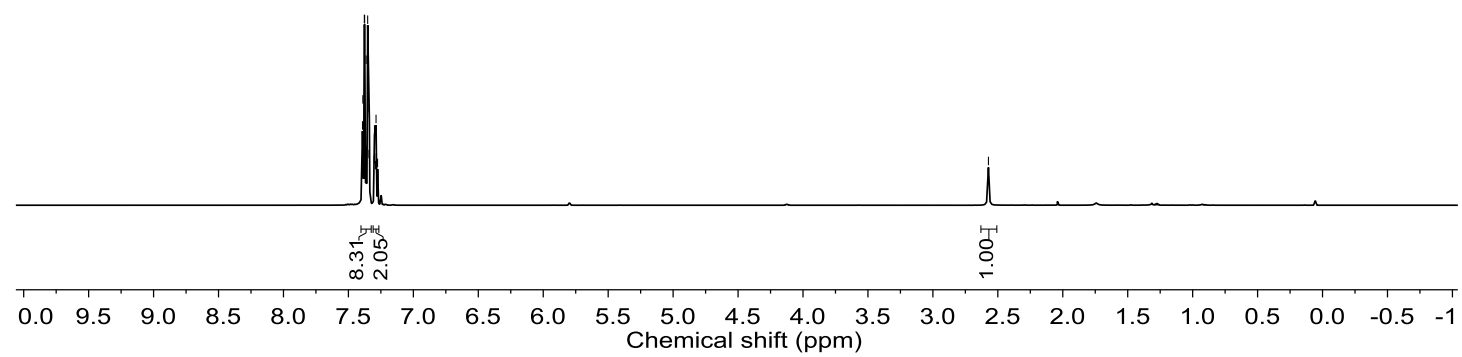<smiles></smiles>

$1 a$

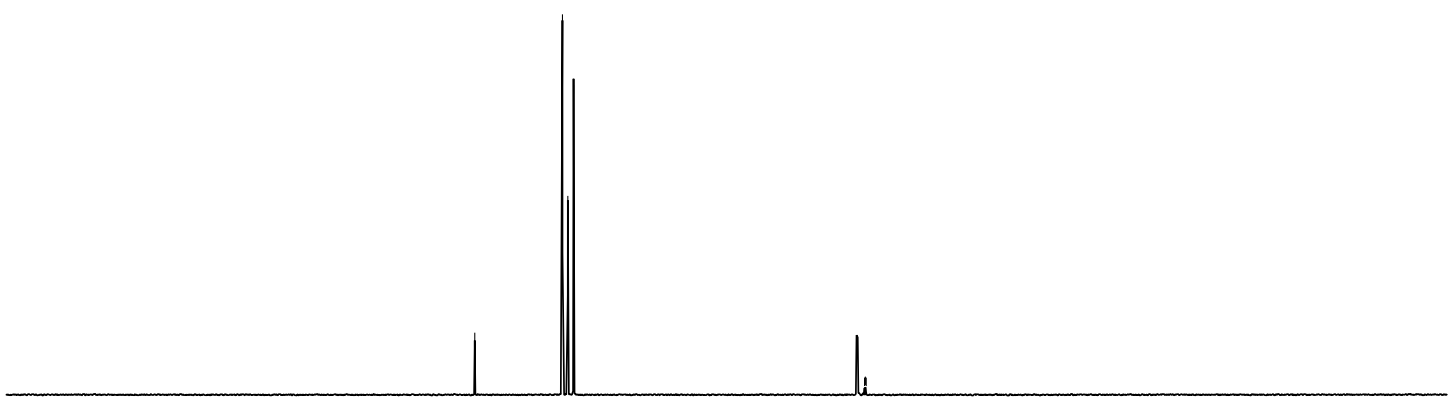

$\begin{array}{llllllllllllllllllllllllll}220 & 210 & 200 & 190 & 180 & 170 & 160 & 150 & 140 & 130 & 120 & 110 & 100 & 90 & 80 & 70 & 60 & 50 & 40 & 30 & 20 & 10 & 0 & -10 & -20\end{array}$ Chemical shift (ppm) 


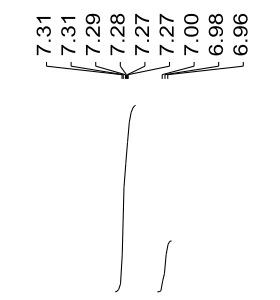<smiles>OC(c1ccccc1)(c1ccc(F)cc1)[14c]1ccccc1</smiles>

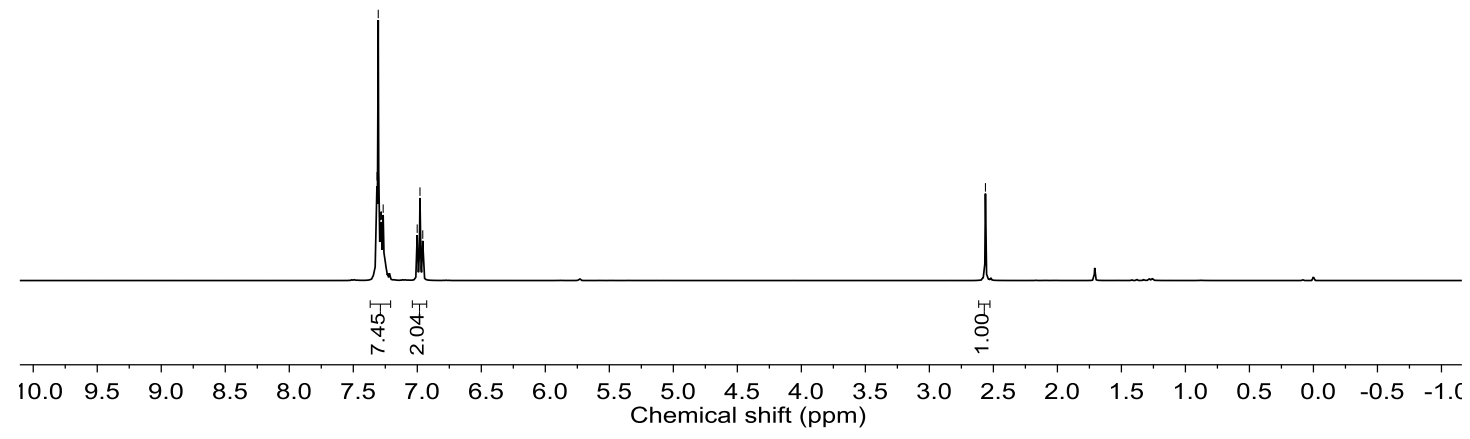<smiles>OC(O)(c1ccccc1)c1ccc(F)cc1</smiles>

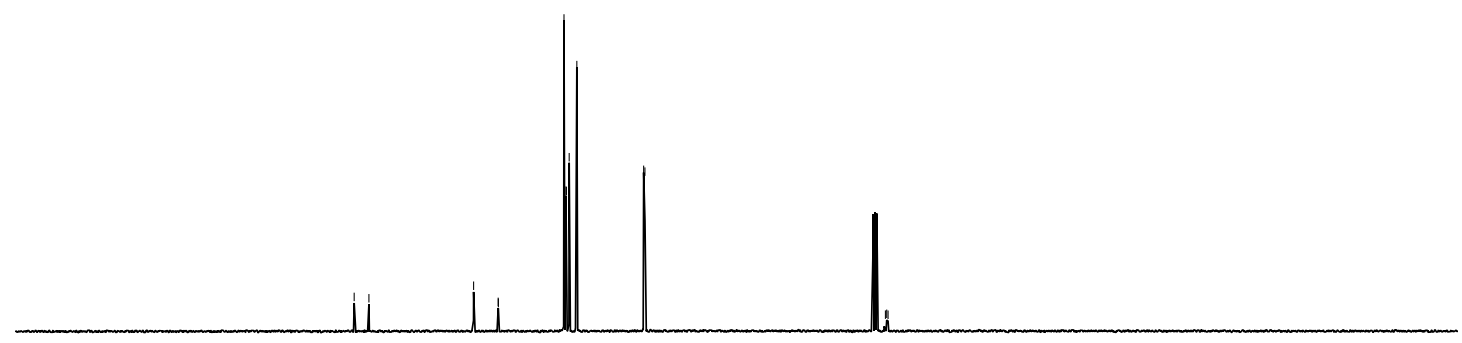

$\begin{array}{llllllllllllllllllllllll}210 & 200 & 190 & 180 & 170 & 160 & 150 & 140 & 130 & 120 & 110 & 100 & 90 & 80 & 70 & 60 & 50 & 40 & 30 & 20 & 10 & 0 & -10\end{array}$ Chemical shift (ppm) 
<smiles>[2H]C(O)(c1ccccc1)c1ccc(F)cc1</smiles>
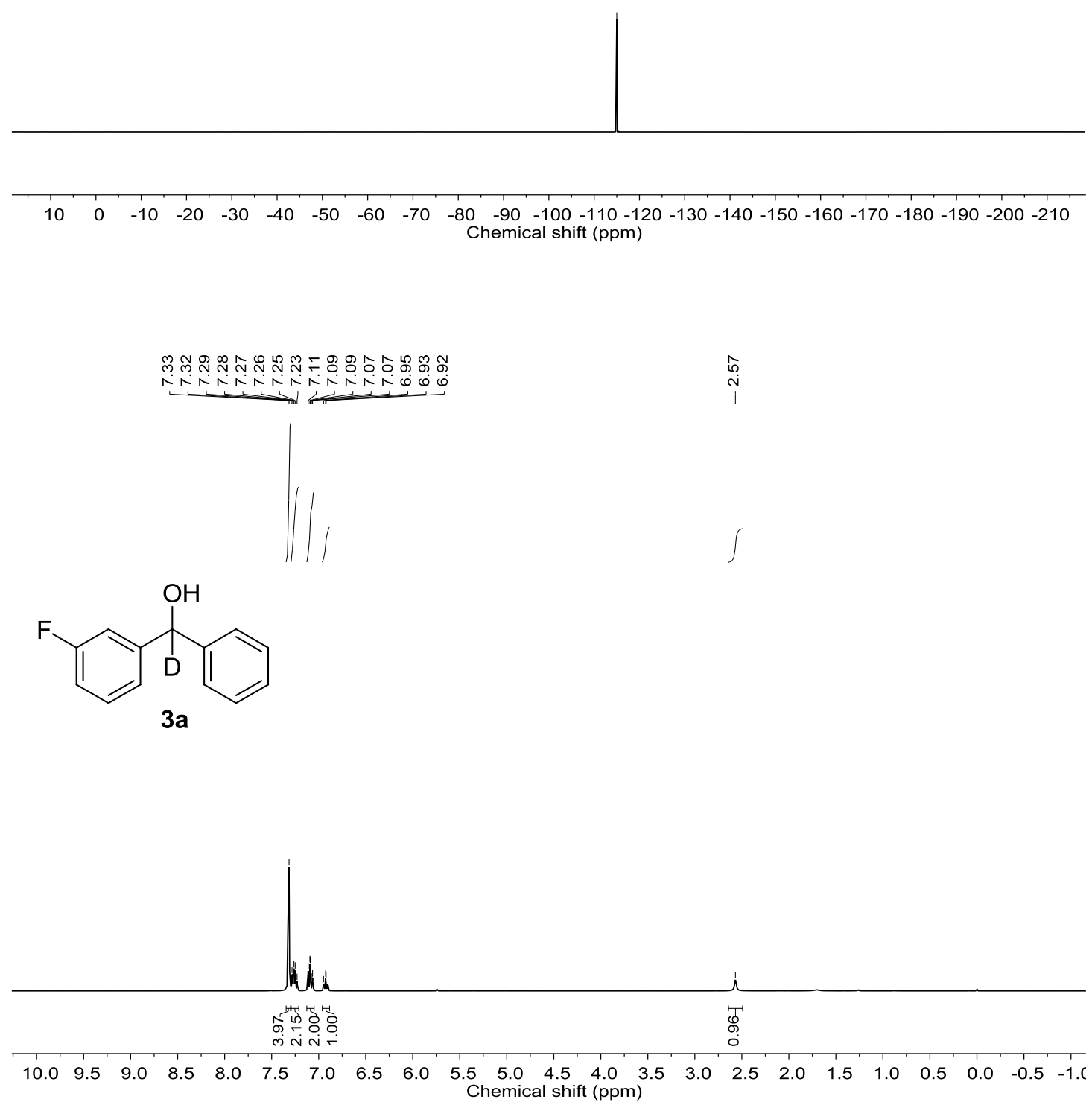


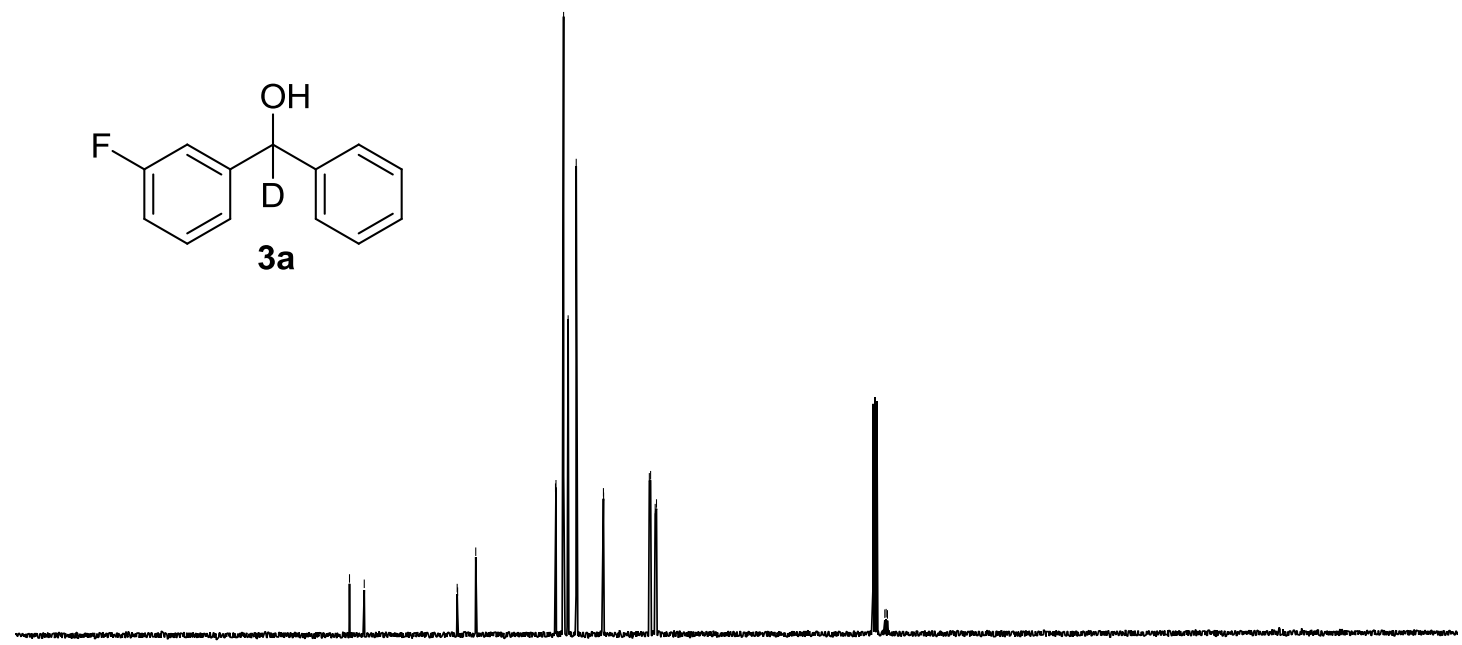

$\begin{array}{llllllllllllllllllllllll}210 & 200 & 190 & 180 & 170 & 160 & 150 & 140 & 130 & 120 & 110 & 100 & 90 & 80 & 70 & 60 & 50 & 40 & 30 & 20 & 10 & 0 & -10\end{array}$ Chemical shift (ppm)<smiles>OC(c1ccccc1)(c1cccc(F)c1)[14c]1ccccc1</smiles>

3a

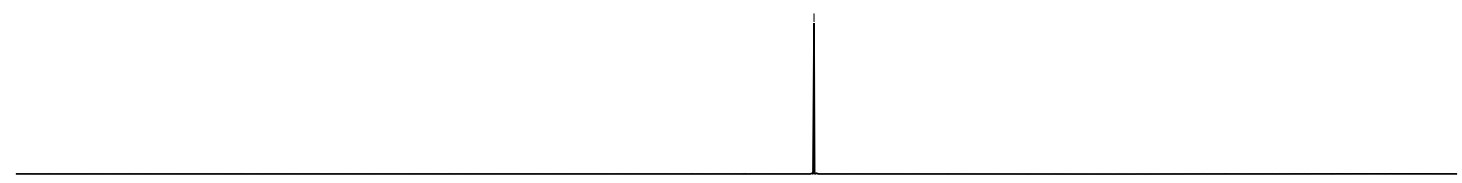

$\begin{array}{llllllllllllllllllllllll}0 & 0 & -10 & -20 & -30 & -40 & -50 & -60 & -70 & -80 & -90 & -100 & -110 & -120 & -130 & -140 & -150 & -160 & -170 & -180 & -190 & -200 & -210\end{array}$ Chemical shift (ppm) 

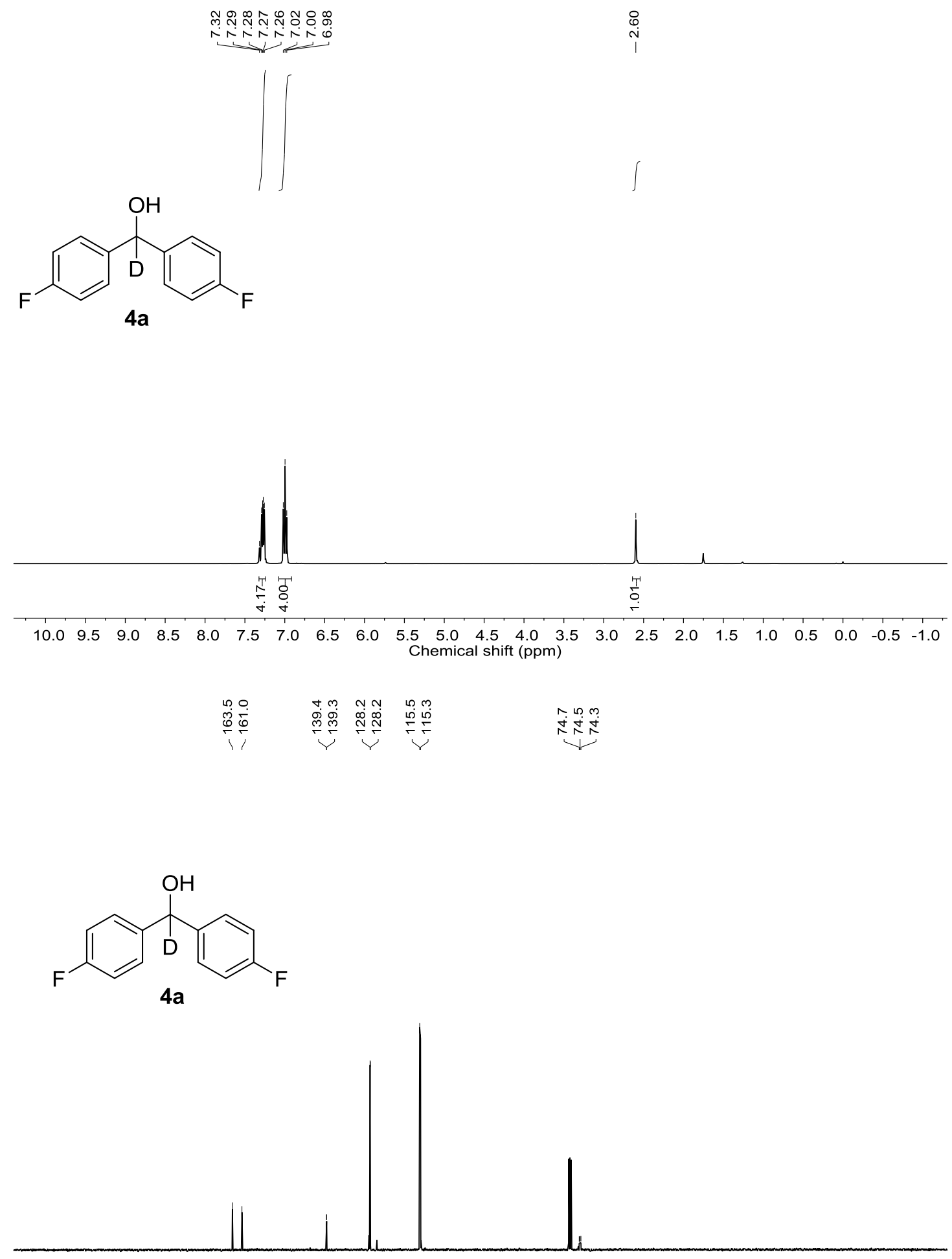

$\begin{array}{llllllllllllllllllllllllllll}210 & 200 & 190 & 180 & 170 & 160 & 150 & 140 & 130 & 120 & 110 & 100 & 90 & 80 & 70 & 60 & 50 & 40 & 30 & 20 & 10 & 0 & -10\end{array}$ Chemical shift (ppm) 
<smiles>[2H]C(O)(c1ccc(F)cc1)c1ccc(F)cc1</smiles>

$4 a$

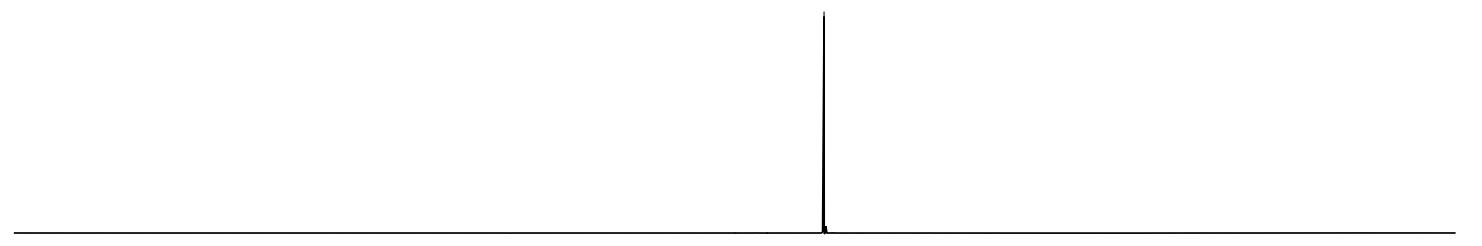

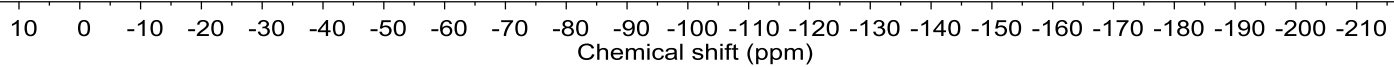

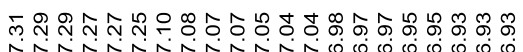

$\stackrel{\bullet}{\stackrel{\bullet}{*}}$

$\int 1$<smiles>[2H]C(O)(c1cccc(F)c1)c1cccc(F)c1</smiles>

$5 a$

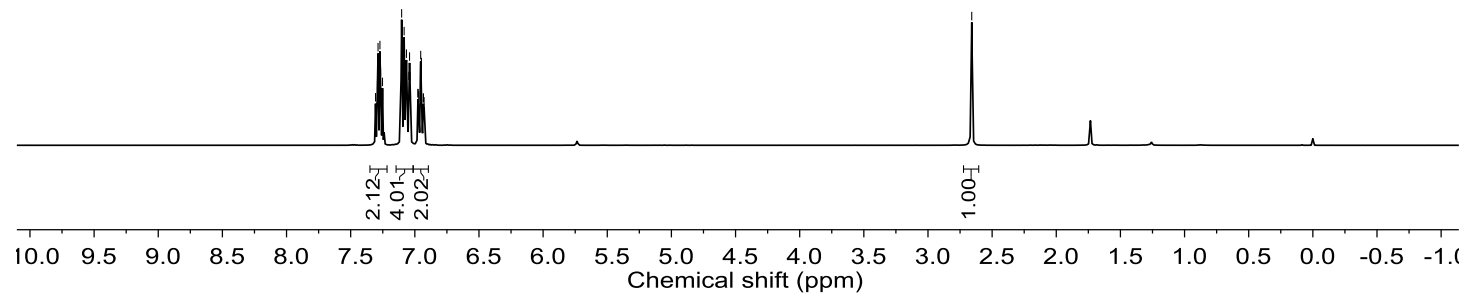


<smiles>OC(c1cccc(F)c1)(c1cccc(F)c1)c1cccc(F)c1</smiles>

$5 a$

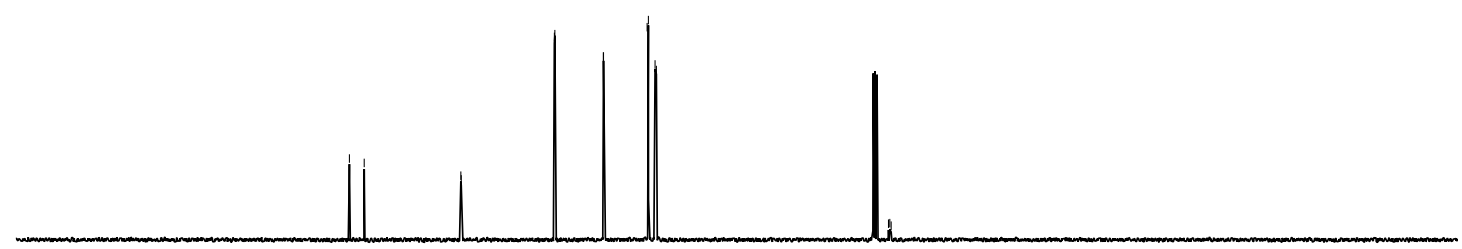

$\begin{array}{llllllllllllllllllllllll}210 & 200 & 190 & 180 & 170 & 160 & 150 & 140 & 130 & 120 & 110 & 100 & 90 & 80 & 70 & 60 & 50 & 40 & 30 & 20 & 10 & 0 & -10\end{array}$ Chemical shift (ppm)<smiles>OC(c1cccc(F)c1)(c1cccc(F)c1)c1cccc(F)c1</smiles>

$5 a$

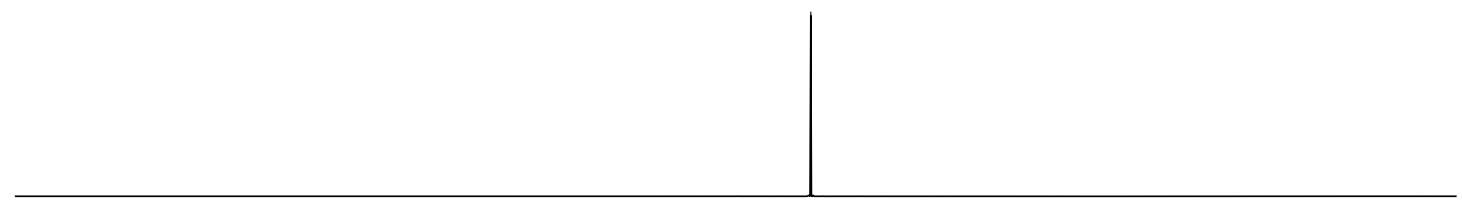

$\begin{array}{lllllllllllllllllllllllll}10 & 0 & -10 & -20 & -30 & -40 & -50 & -60 & -70 & -80 & -90 & -100 & -110 & -120 & -130 & -140 & -150 & -160 & -170 & -180 & -190 & -200 & -210\end{array}$ Chemical shift (ppm) 


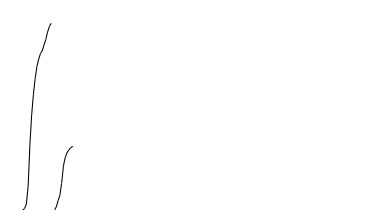<smiles>[2H]C(O)(c1ccccc1)c1ccc(F)c(F)c1</smiles>

$6 a$

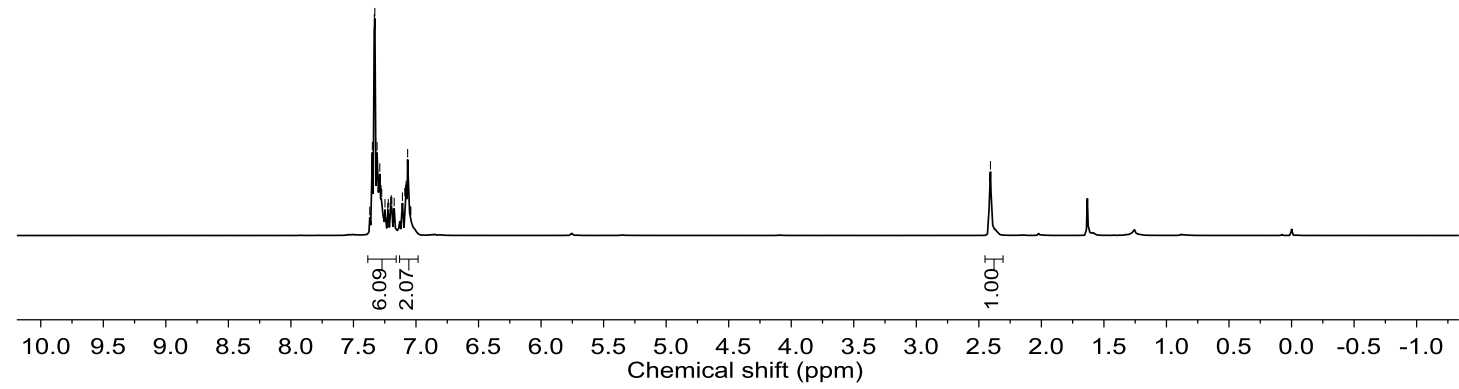
$\underbrace{0}$<smiles>[2H]C(O)(c1ccccc1)c1ccc(F)c(F)c1</smiles>

$6 a$

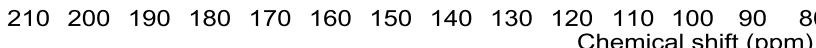


<smiles>OC(c1ccccc1)(c1ccccc1)c1ccc(F)c(F)c1</smiles>

$6 a$

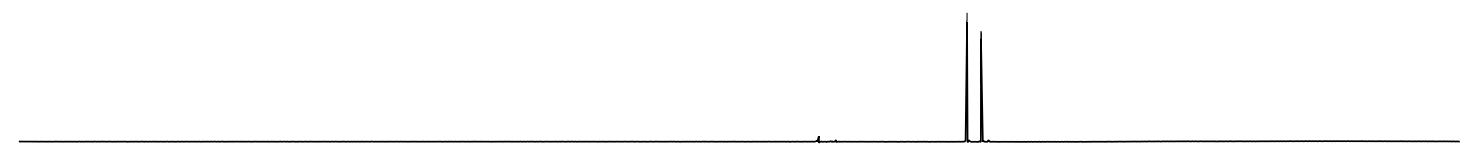

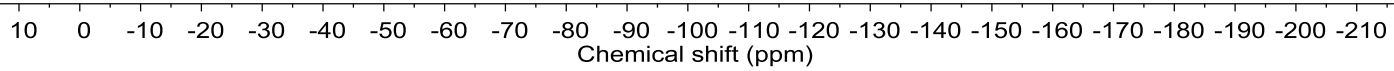

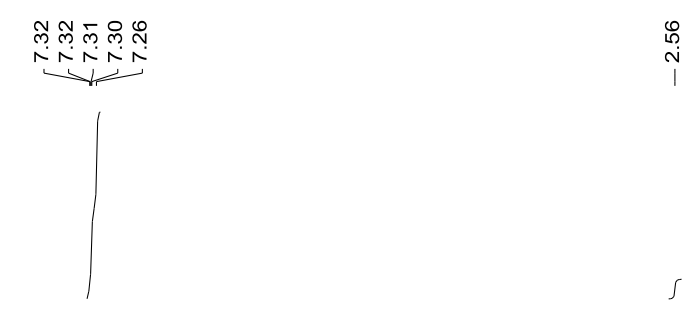<smiles>[2H]C(O)(c1ccccc1)c1ccc(Cl)cc1</smiles>

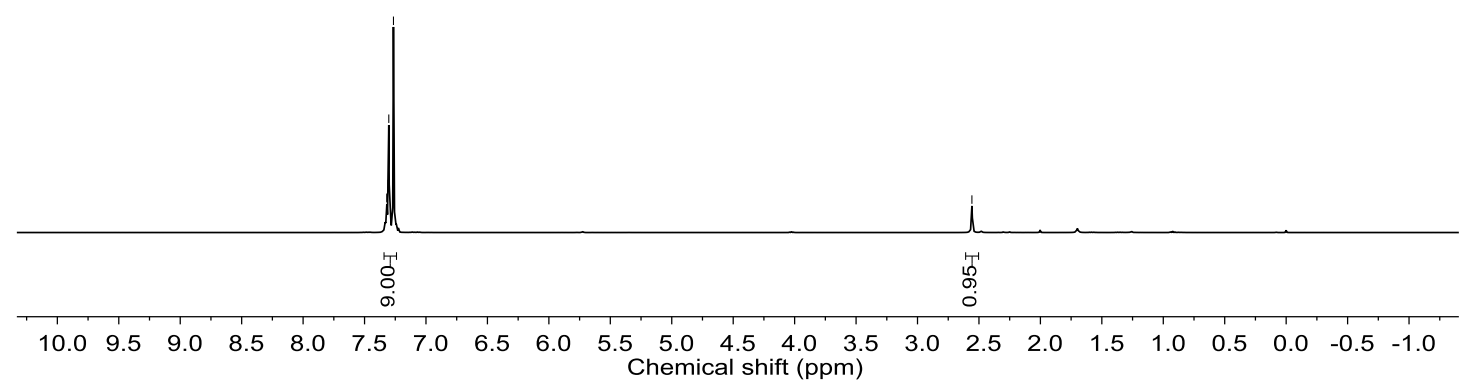


(c)

$7 a$

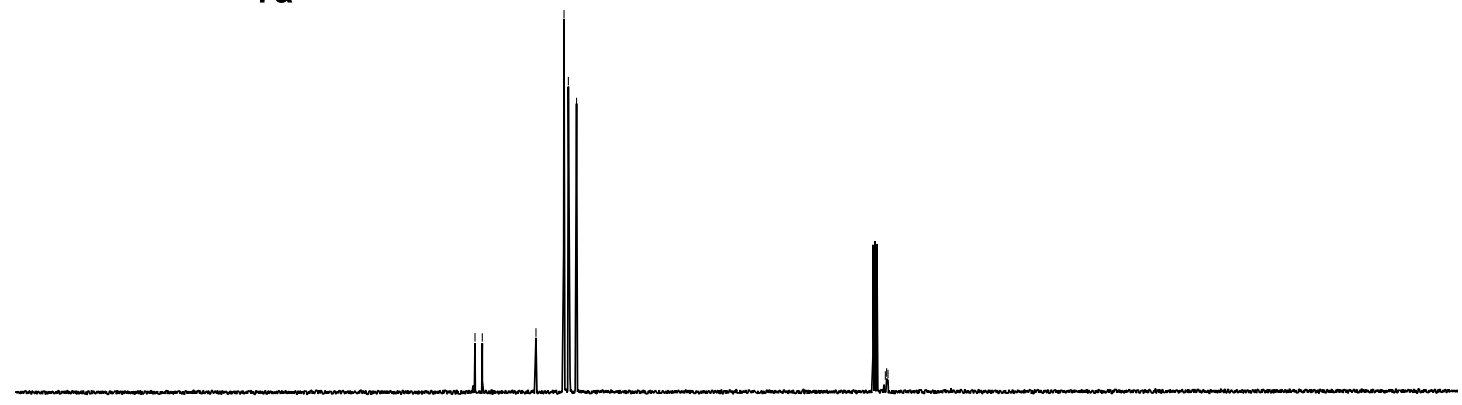

$\begin{array}{llllllllllllllllllllllll}210 & 200 & 190 & 180 & 170 & 160 & 150 & 140 & 130 & 120 & 110 & 100 & 90 & 80 & 70 & 60 & 50 & 40 & 30 & 20 & 10 & 0 & -10\end{array}$ Chemical shift (ppm)

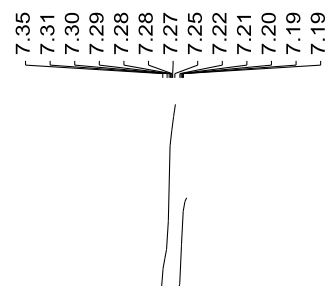<smiles>[2H]C(O)(c1ccccc1)c1cccc(Cl)c1</smiles>

$8 a$

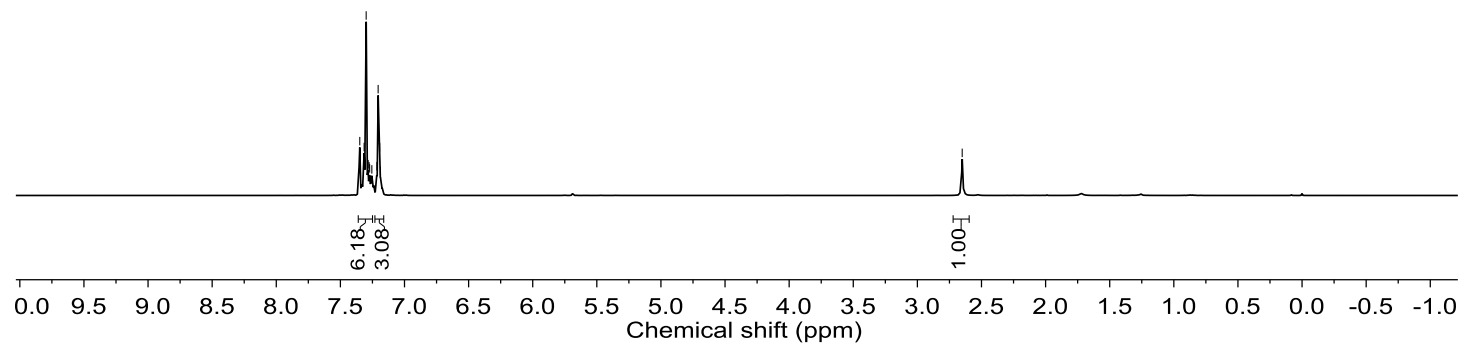


<smiles>[2H]C(O)(c1ccccc1)c1cccc(Cl)c1</smiles>

$8 a$

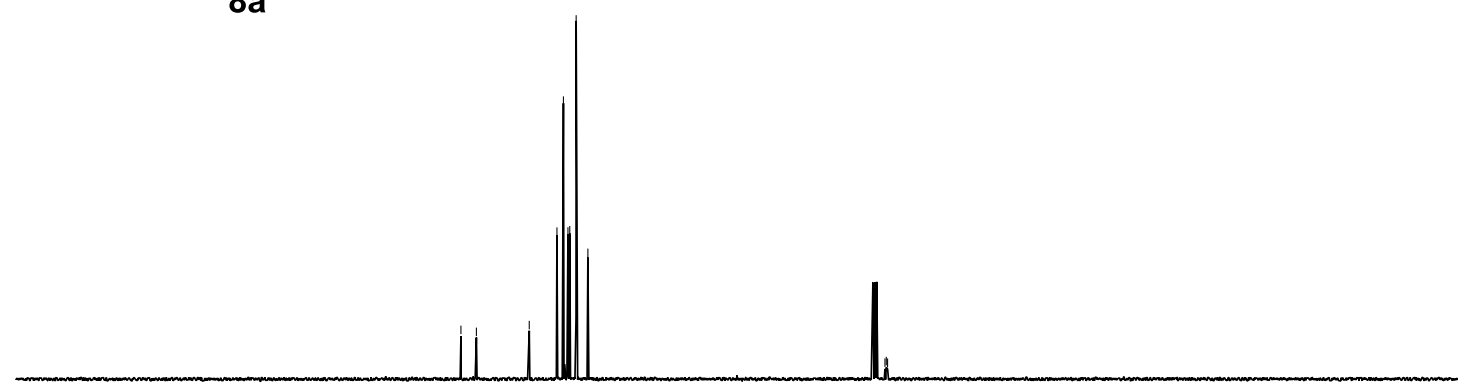

$\begin{array}{lllllllllllllllllllllll}210 & 200 & 190 & 180 & 170 & 160 & 150 & 140 & 130 & 120 & 110 & 100 & 90 & 80 & 70 & 60 & 50 & 40 & 30 & 20 & 10 & 0 & -10\end{array}$ Chemical shift (ppm)
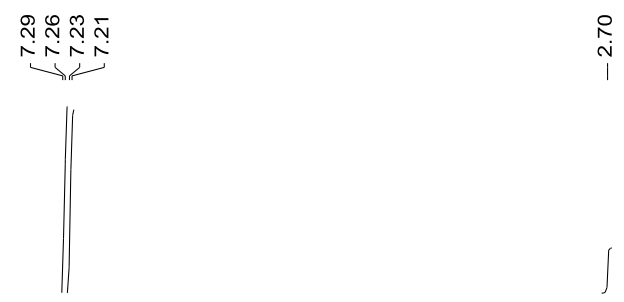<smiles>OC(O)(c1ccc(Cl)cc1)c1ccc(Cl)cc1</smiles>

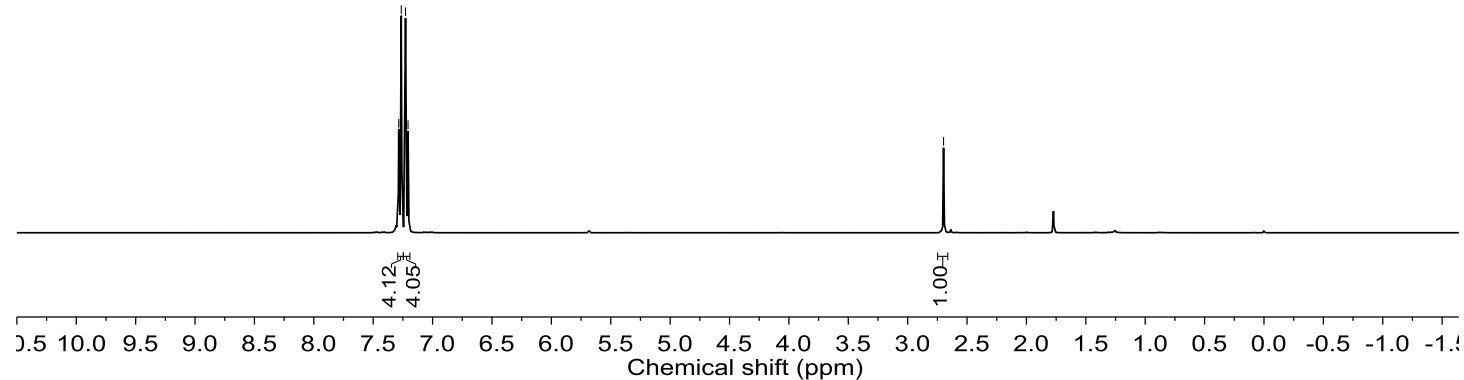


<smiles>[2H]C(O)(c1ccc(Cl)cc1)c1ccc(Cl)cc1</smiles>

9a

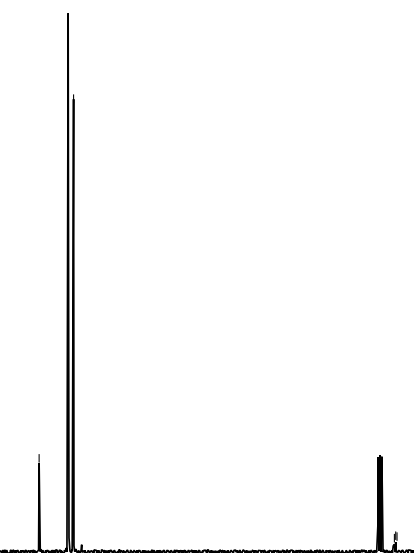

$\begin{array}{lllllllllllllllllllllll}210 & 200 & 190 & 180 & 170 & 160 & 150 & 140 & 130 & 120 & 110 & 100 & 90 & 80 & 70 & 60 & 50 & 40 & 30 & 20 & 10 & 0 & -10\end{array}$ Chemical shift (ppm)

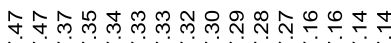

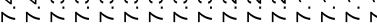

1<smiles>OC(O)(c1ccccc1)c1ccc(Cl)c(Cl)c1</smiles>

$10 \mathrm{a}$

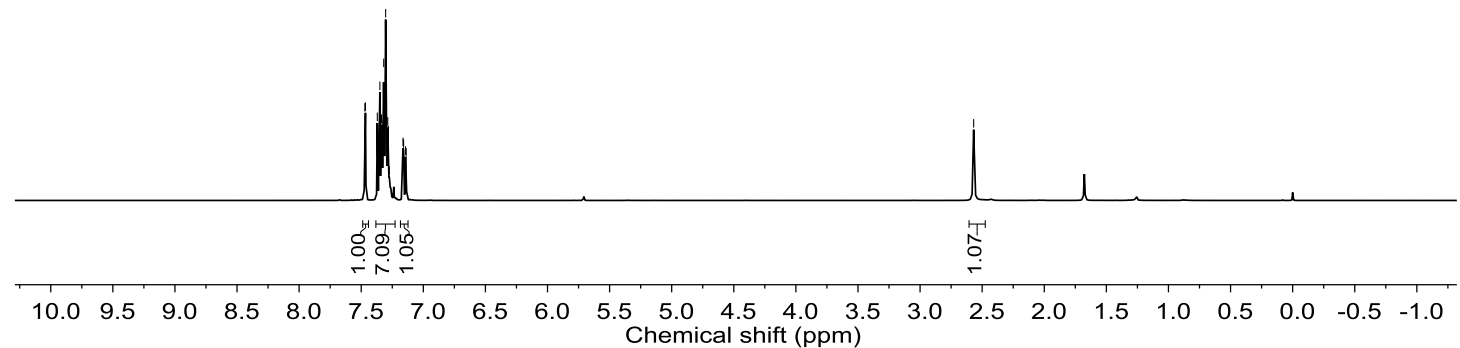


<smiles>[2H]C(O)(c1ccccc1)c1ccc(Cl)c(Cl)c1</smiles>

$\begin{array}{lllllllllllllllllllllll}210 & 200 & 190 & 180 & 170 & 160 & 150 & 140 & 130 & 120 & 110 & 100 & 90 & 80 & 70 & 60 & 50 & 40 & 30 & 20 & 10 & 0 & -10\end{array}$ Chemical shift (ppm)

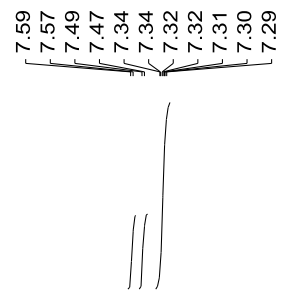<smiles>[2H]C(O)(c1ccccc1)c1ccc(C#N)cc1</smiles>

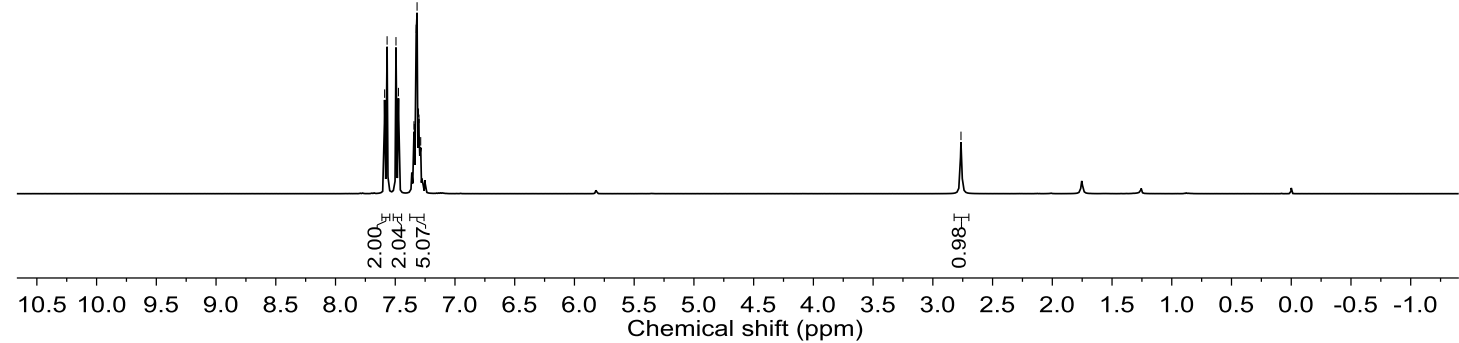




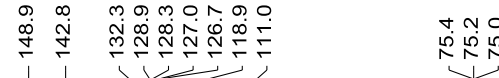<smiles>N#Cc1ccc(C(O)(O)c2ccccc2)cc1</smiles>

$11 a$

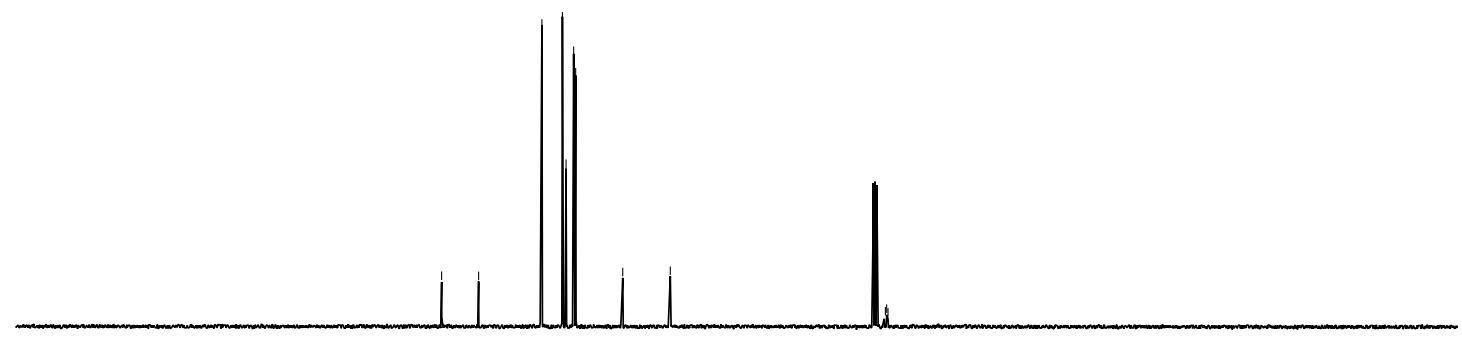

$\begin{array}{lllllllllllllllllllllll}210 & 200 & 190 & 180 & 170 & 160 & 150 & 140 & 130 & 120 & 110 & 100 & 90 & 80 & 70 & 60 & 50 & 40 & 30 & 20 & 10 & 0 & -10\end{array}$ Chemical shift (ppm)
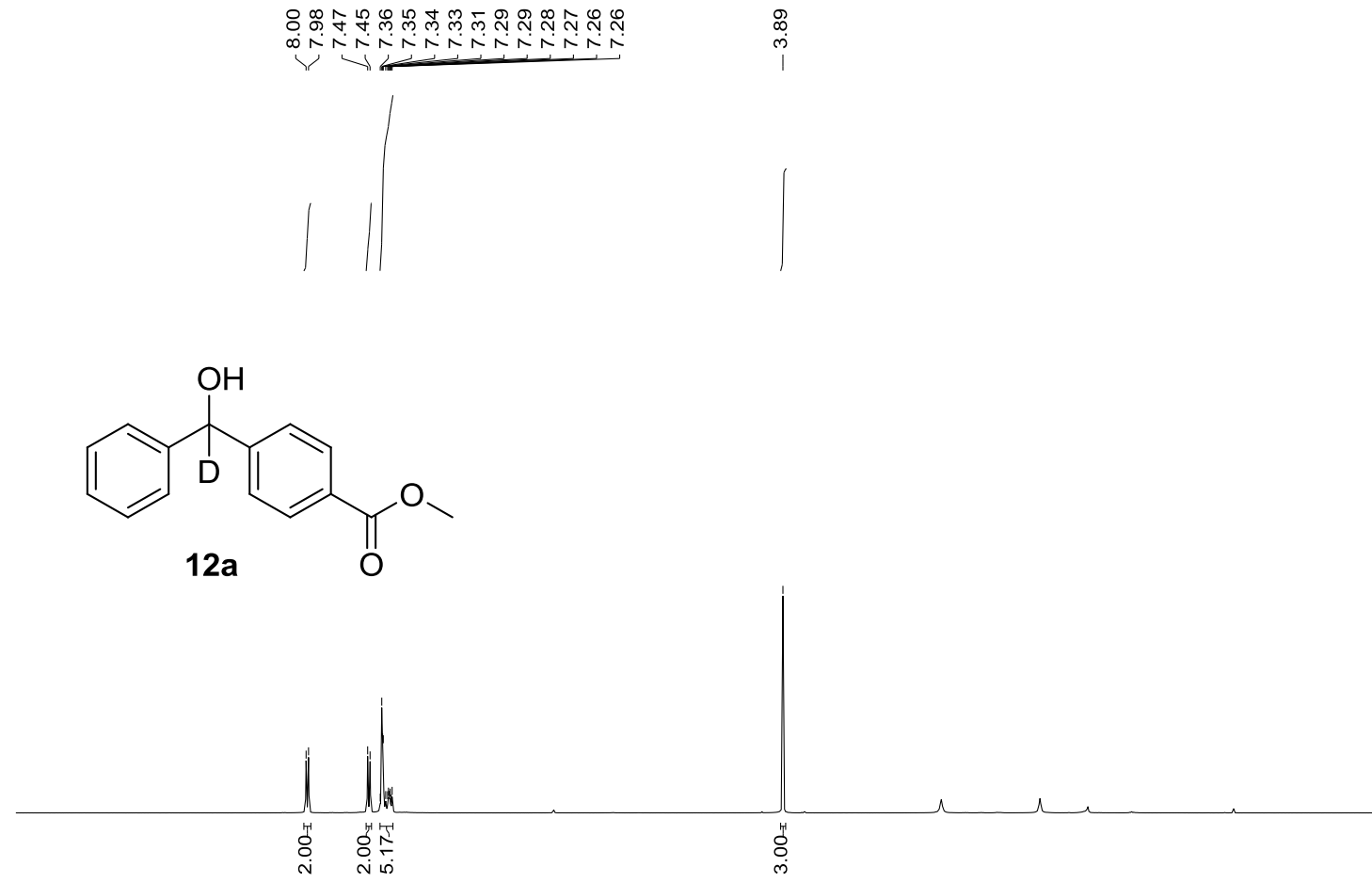

$\begin{array}{llllllllllllllllllllllll}3.5 & 10.0 & 9.5 & 9.0 & 8.5 & 8.0 & 7.5 & 7.0 & 6.5 & 6.0 & 5.5 & 5.0 & 4.5 & 4.0 & 3.5 & 3.0 & 2.5 & 2.0 & 1.5 & 1.0 & 0.5 & 0.0 & -0.5 & -1.0\end{array}$ Chemical shift (ppm) 


l

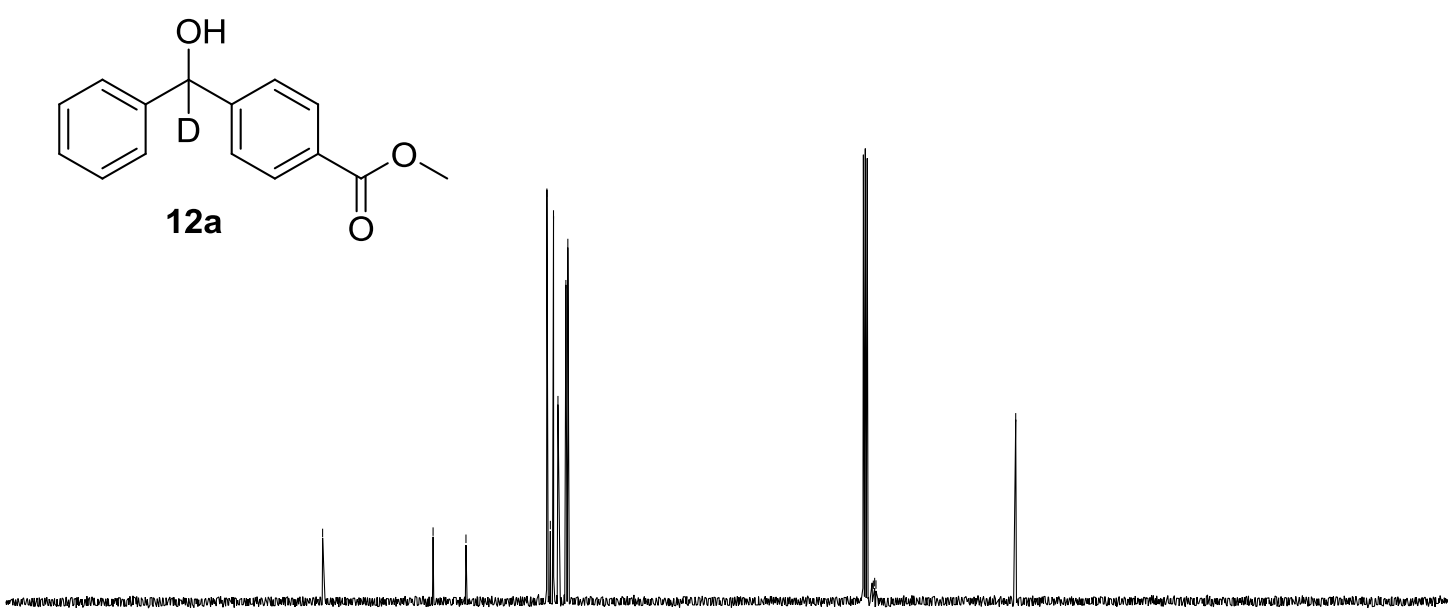

$\begin{array}{lllllllllllllllllllllll}210 & 200 & 190 & 180 & 170 & 160 & 150 & 140 & 130 & 120 & 110 & 100 & 90 & 80 & 70 & 60 & 50 & 40 & 30 & 20 & 10 & 0 & -10\end{array}$

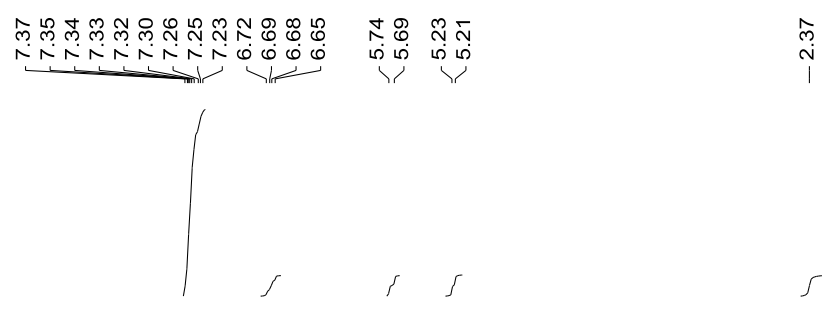<smiles>[2H]C(O)(c1ccccc1)c1ccc(C=C)cc1</smiles>

$13 a$

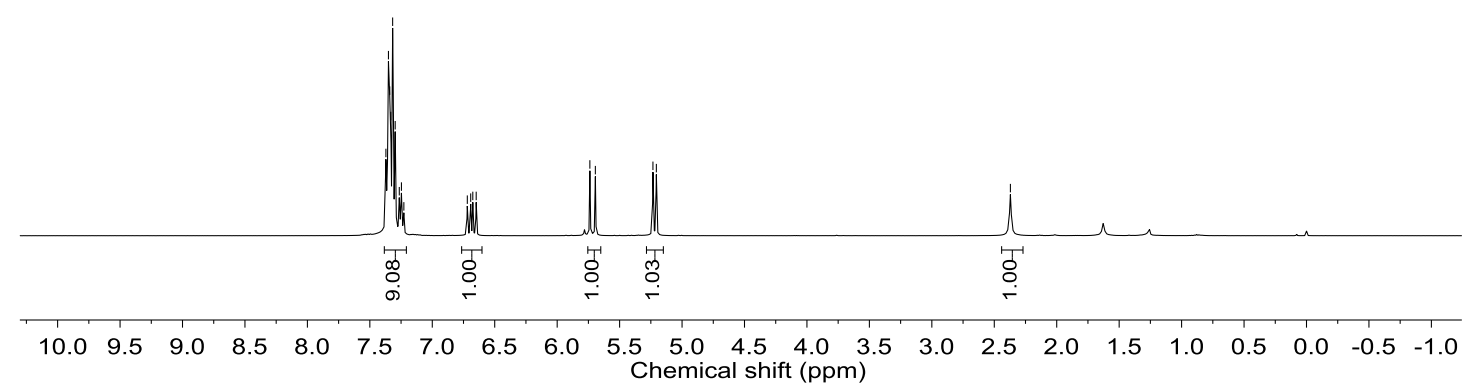


<smiles>[2H]C(O)(c1ccccc1)c1ccc(C=C)cc1</smiles>

$13 a$

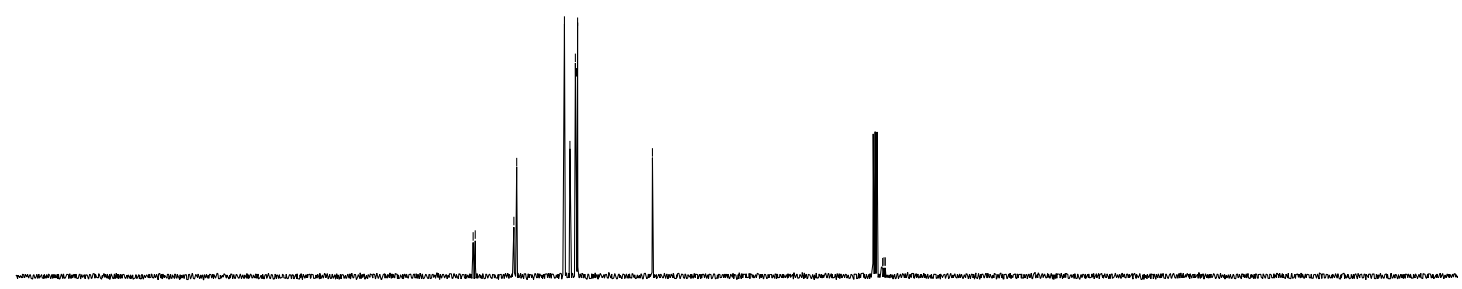

$\begin{array}{lllllllllllllllllllllll}210 & 200 & 190 & 180 & 170 & 160 & 150 & 140 & 130 & 120 & 110 & 100 & 90 & 80 & 70 & 60 & 50 & 40 & 30 & 20 & 10 & 0 & -10\end{array}$ Chemical shift (ppm)
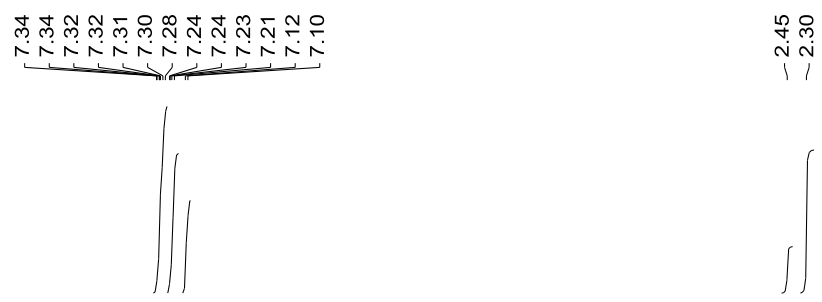<smiles>[2H]C(O)(c1ccccc1)c1ccc(C)cc1</smiles>

$14 a$

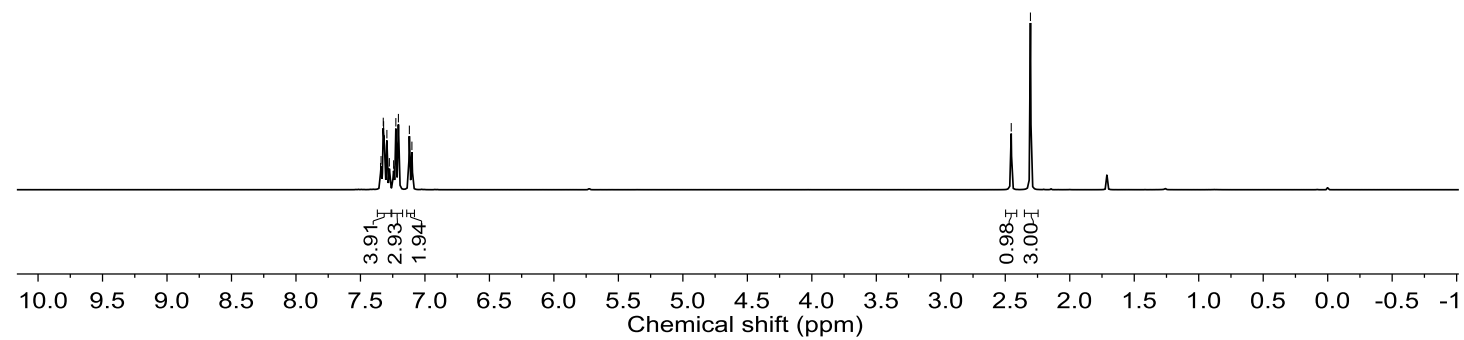


<smiles>Cc1ccc(C(O)(c2ccccc2)c2ccccc2)cc1</smiles>

$14 a$
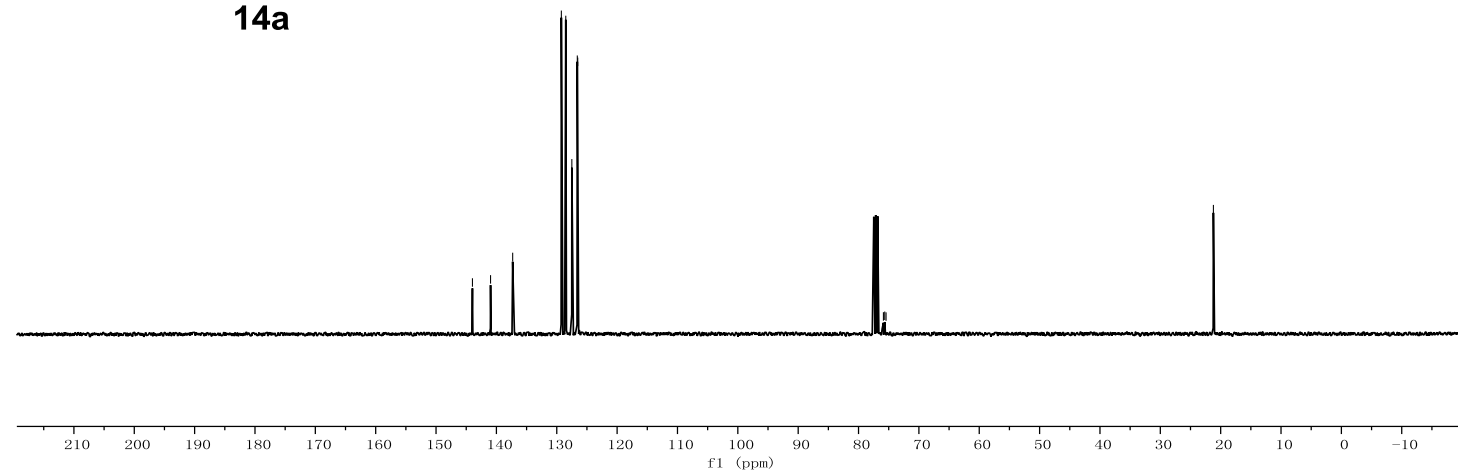

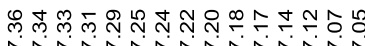

\%̊ำ

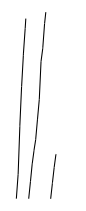

$\sqrt{3}$

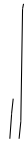

$N_{D}^{O H}$

$15 a$

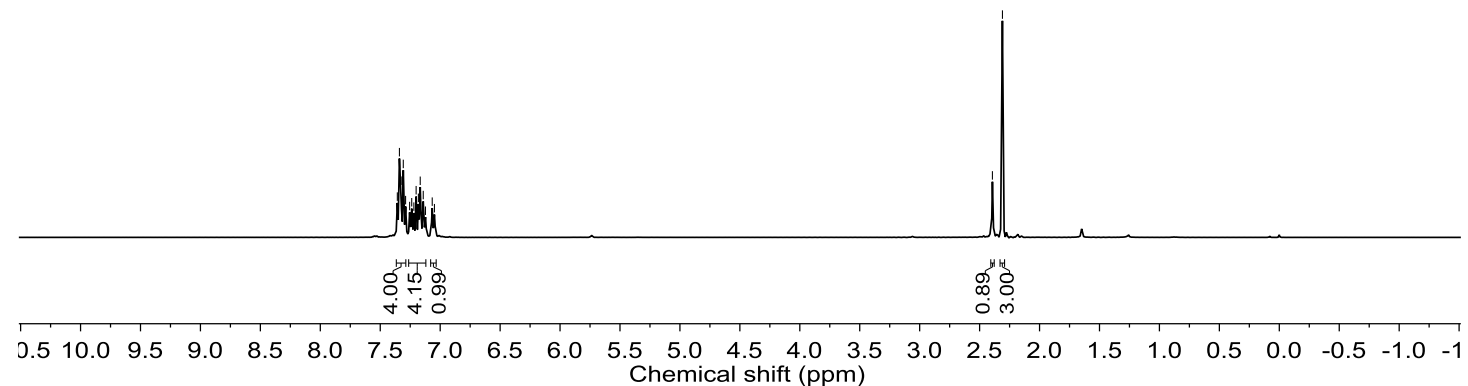


<smiles>[2H]C(O)(c1ccccc1)c1cccc(C)c1</smiles>

$15 a$

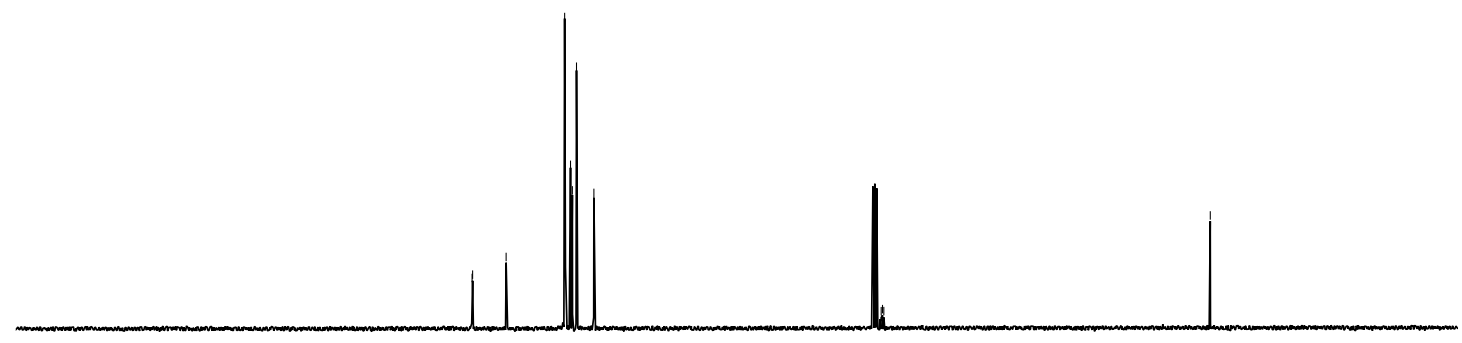

$\begin{array}{lllllllllllllllllllllll}210 & 200 & 190 & 180 & 170 & 160 & 150 & 140 & 130 & 120 & 110 & 100 & 90 & 80 & 70 & 60 & 50 & 40 & 30 & 20 & 10 & 0 & -10\end{array}$ Chemical shift (ppm)

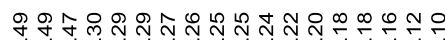

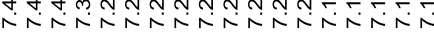

$\stackrel{\ln }{\stackrel{N}{N}}$

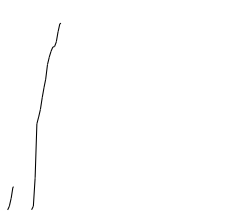

ก<smiles>[2H]C(O)(c1ccccc1)c1ccccc1C</smiles>

$16 \mathrm{a}$

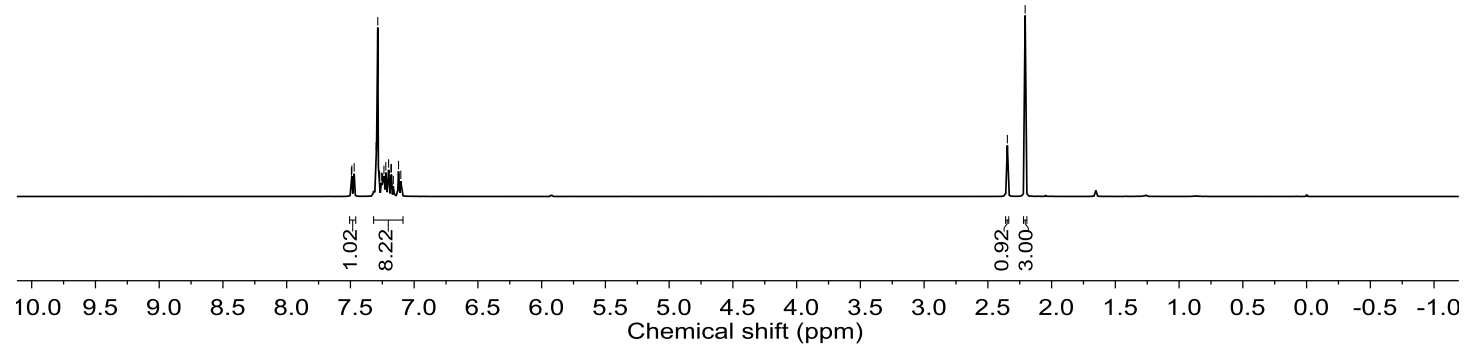


<smiles>Cc1ccccc1C(O)(O)c1ccccc1</smiles>

$16 a$

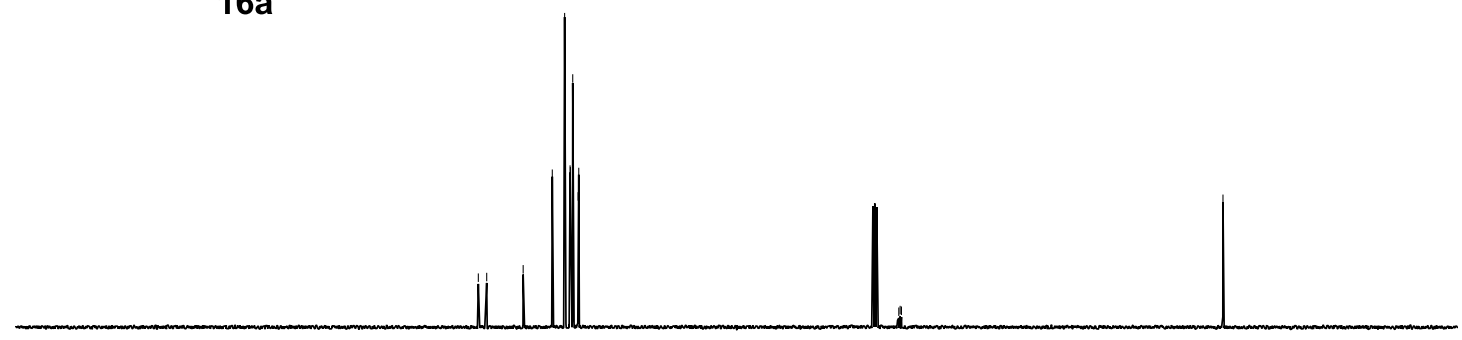

$\begin{array}{lllllllllllllllllllllll}210 & 200 & 190 & 180 & 170 & 160 & 150 & 140 & 130 & 120 & 110 & 100 & 90 & 80 & 70 & 60 & 50 & 40 & 30 & 20 & 10 & 0 & -10\end{array}$ Chemical shift (ppm)

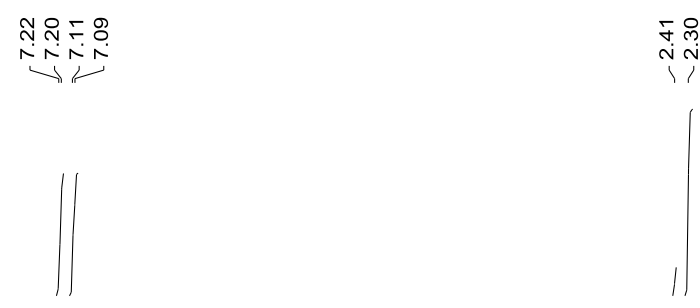<smiles>Cc1ccc(C(O)(c2ccc(C)cc2)c2ccc(C)cc2)cc1</smiles>

$17 a$

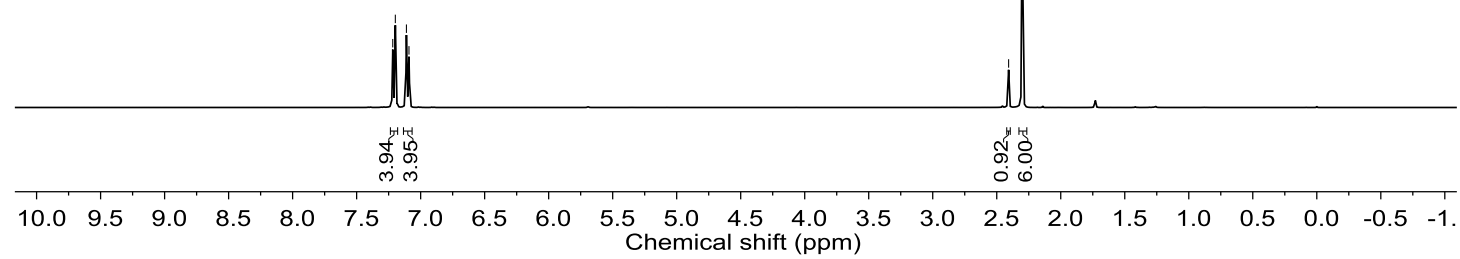




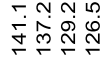

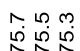

$\sqrt{5}$

$\stackrel{\text { ก }}{4}$

$N_{D}^{O H}$

$17 a$

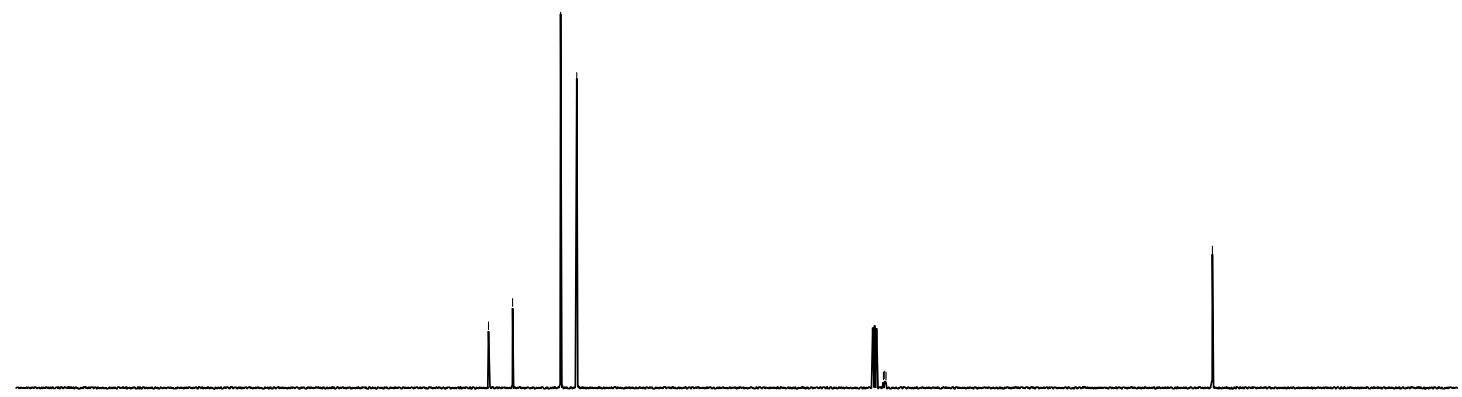

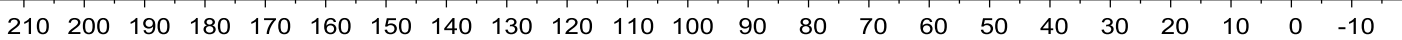
Chemical shift (ppm)

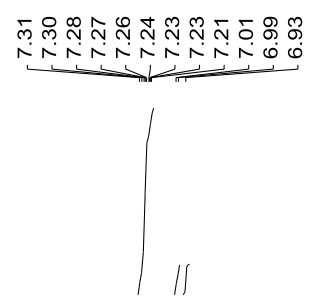

พูำ ำ

$\sin$

$\|$<smiles>Cc1ccc(C)c(C(O)(O)c2ccccc2)c1</smiles>

$18 a$

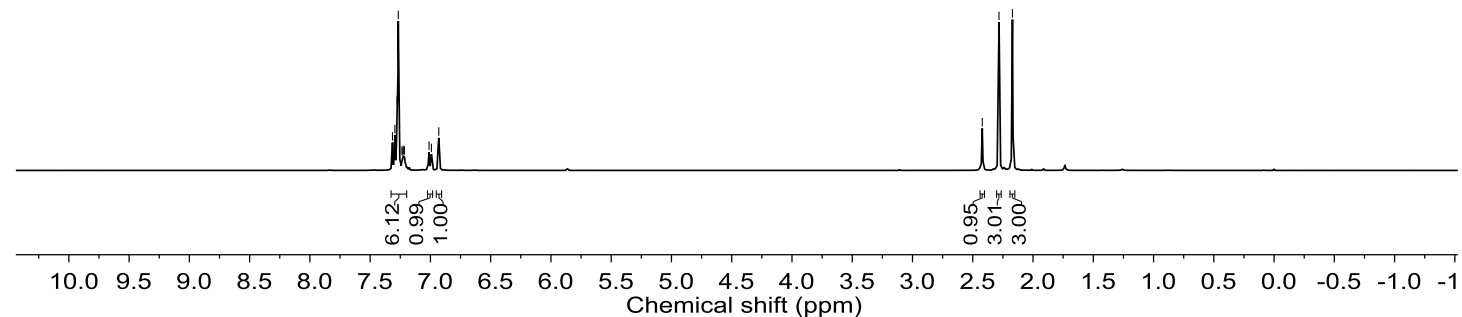


<smiles>Cc1ccc(C)c(C(O)(c2ccccc2)c2ccccc2)c1</smiles>

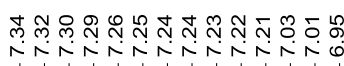

| ||<smiles>[2H]C(O)(c1ccccc1)c1ccc(C)cc1C</smiles>

19a

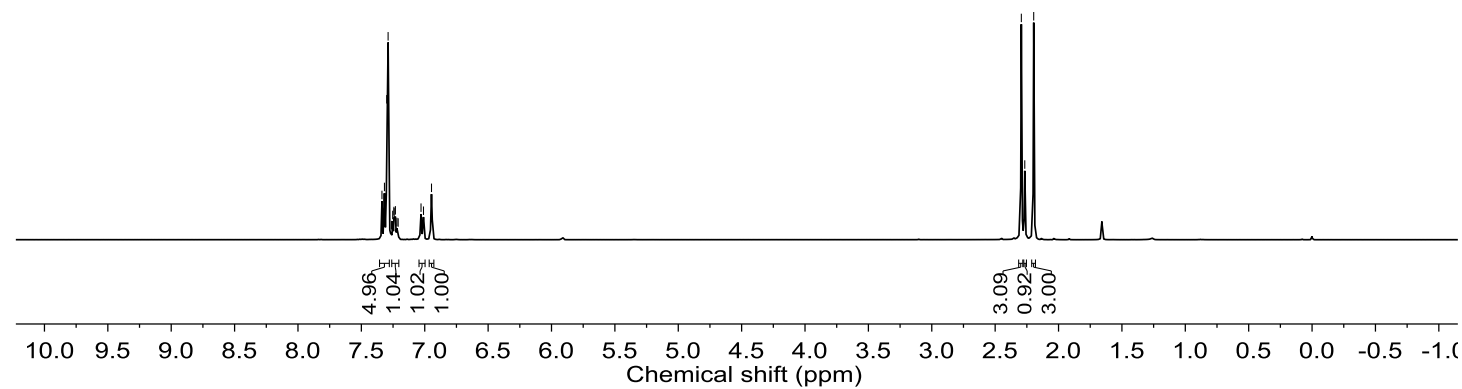


<smiles>[2H]C(O)(c1ccccc1)c1ccc(C)cc1C</smiles>

19a

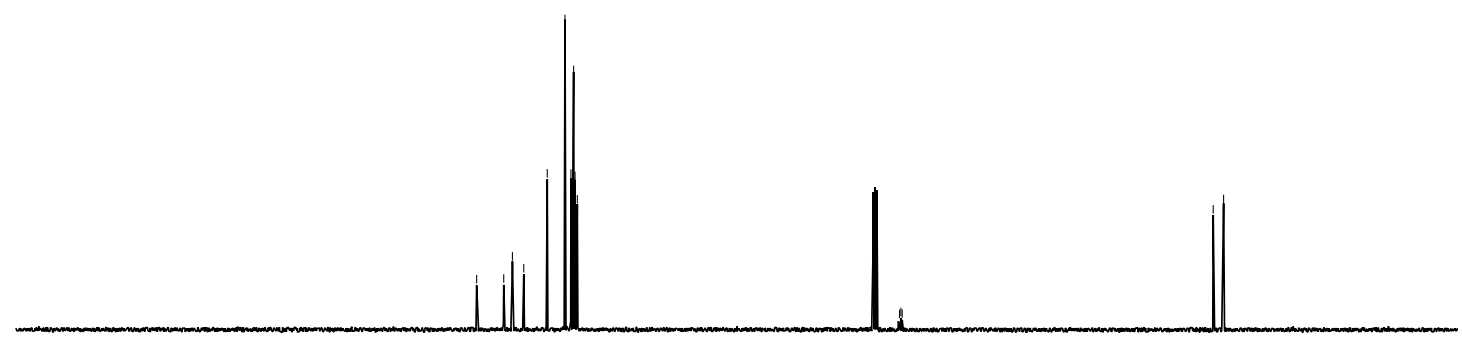

$\begin{array}{lllllllllllllllllllllll}210 & 200 & 190 & 180 & 170 & 160 & 150 & 140 & 130 & 120 & 110 & 100 & 90 & 80 & 70 & 60 & 50 & 40 & 30 & 20 & 10 & 0 & -10\end{array}$ Chemical shift (ppm)

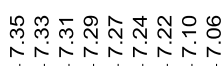

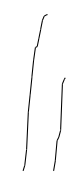

$20 a$<smiles>Cc1ccc(C(O)(O)c2ccccc2)cc1C</smiles>

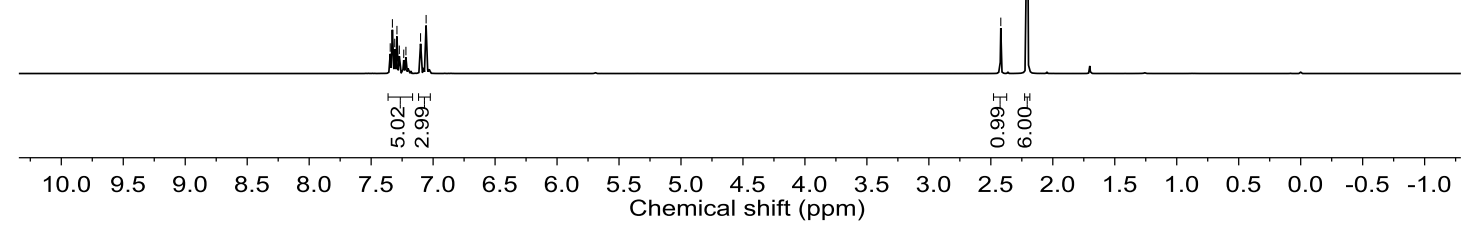


<smiles>Cc1ccc(C(O)(O)c2ccccc2)cc1C</smiles>

20a

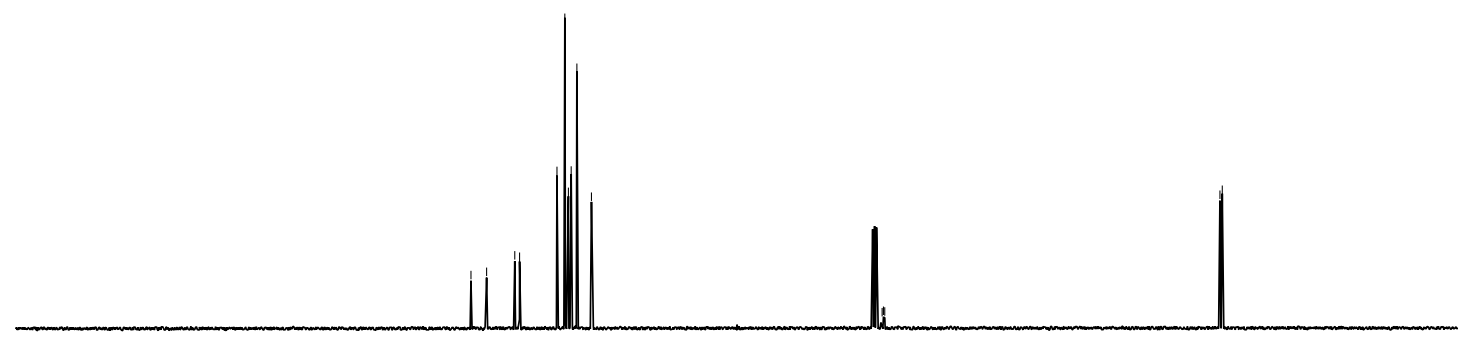

$\begin{array}{lllllllllllllllllllllll}210 & 200 & 190 & 180 & 170 & 160 & 150 & 140 & 130 & 120 & 110 & 100 & 90 & 80 & 70 & 60 & 50 & 40 & 30 & 20 & 10 & 0 & -10\end{array}$ Chemical shift (ppm)

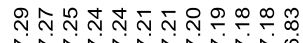

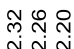

nNankNiNión
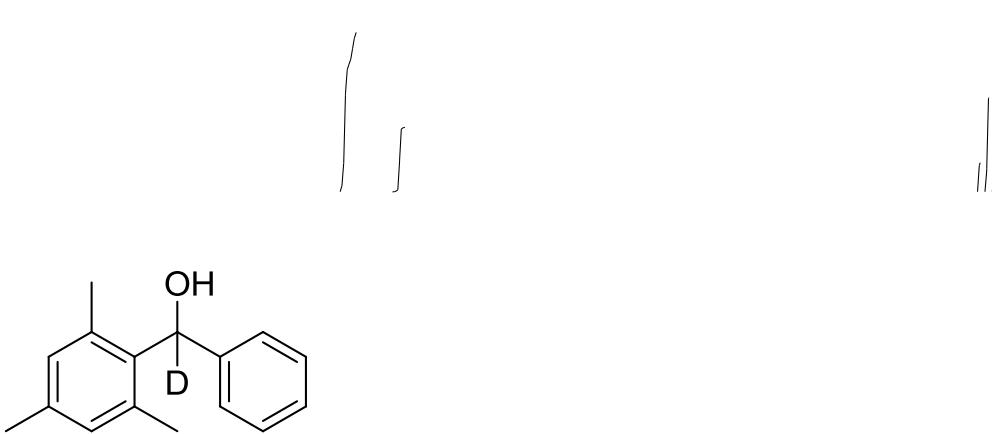

21a

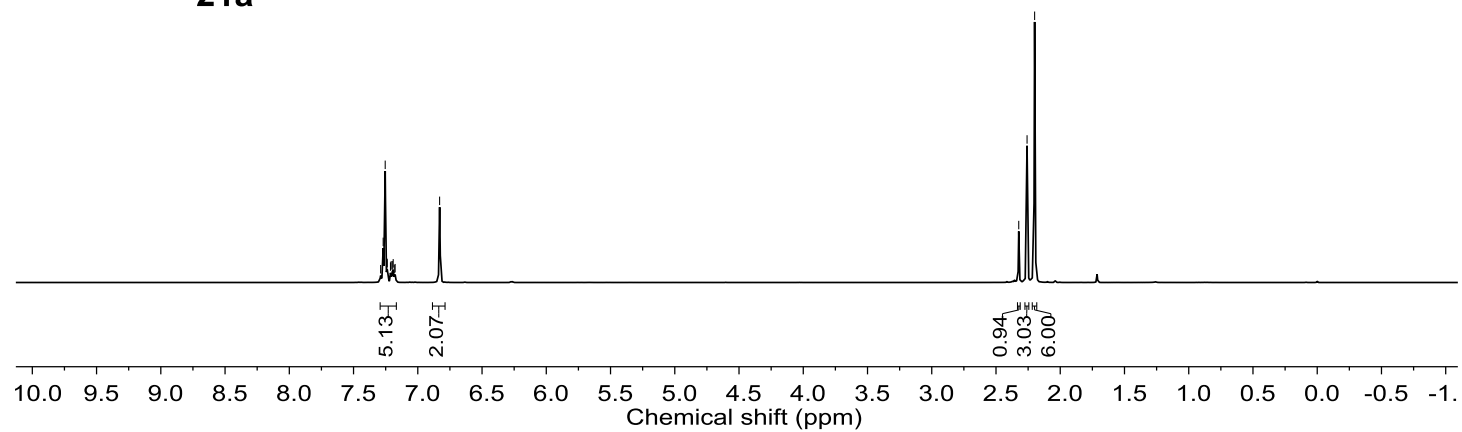


<smiles>[2H]C(O)(c1ccccc1)c1c(C)cc(C)cc1C</smiles>

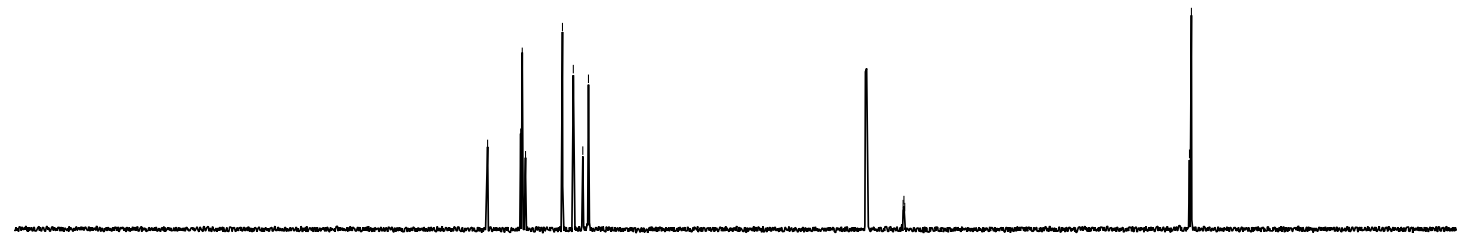

$220210200190180170160 \begin{array}{lllllllllllllllllll} & 150 & 140 & 130 & 120 & 110 & 100 & 90 & 80 & 70 & 60 & 50 & 40 & 30 & 20 & 10 & 0 & -10 & -20\end{array}$ Chemical shift (ppm)

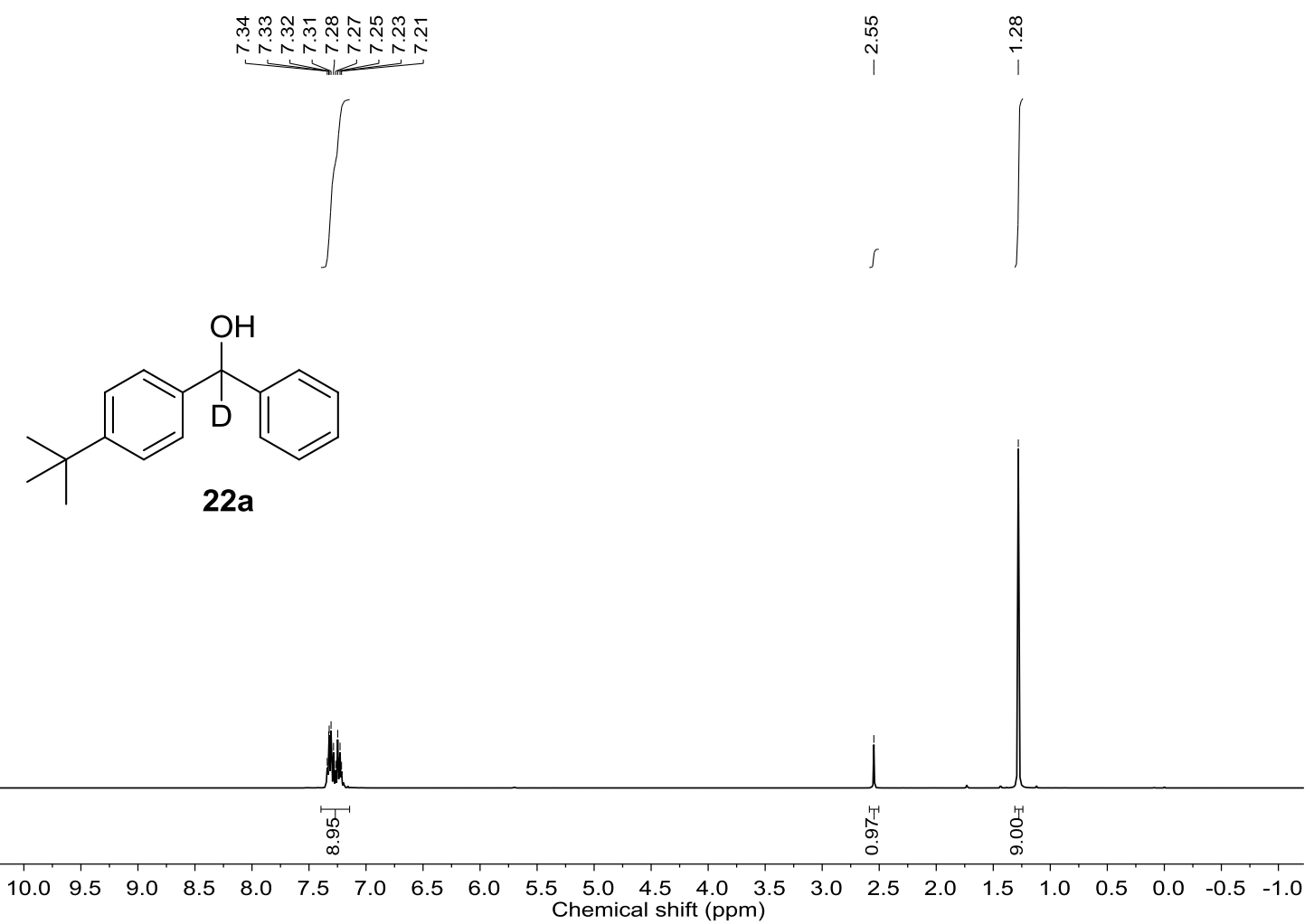


<smiles>[2H]C(O)(c1ccccc1)c1ccc(C(C)(C)C)cc1</smiles>

22a

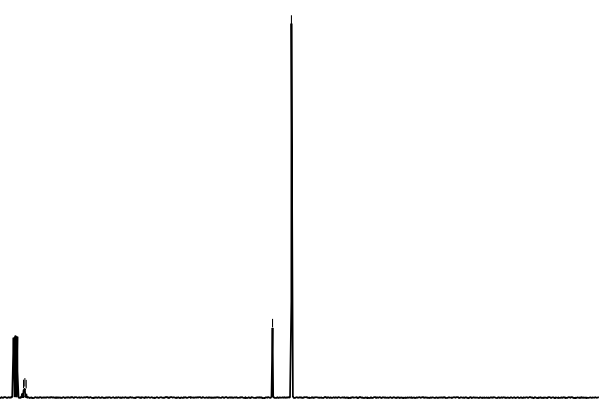

$\begin{array}{lllllllllllllllllllllll}210 & 200 & 190 & 180 & 170 & 160 & 150 & 140 & 130 & 120 & 110 & 100 & 90 & 80 & 70 & 60 & 50 & 40 & 30 & 20 & 10 & 0 & -10\end{array}$ Chemical shift (ppm)

นூم

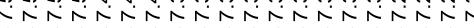

||l

ํำ<smiles>[2H]C(O)(c1ccccc1)c1ccc(-c2ccccc2)cc1</smiles>

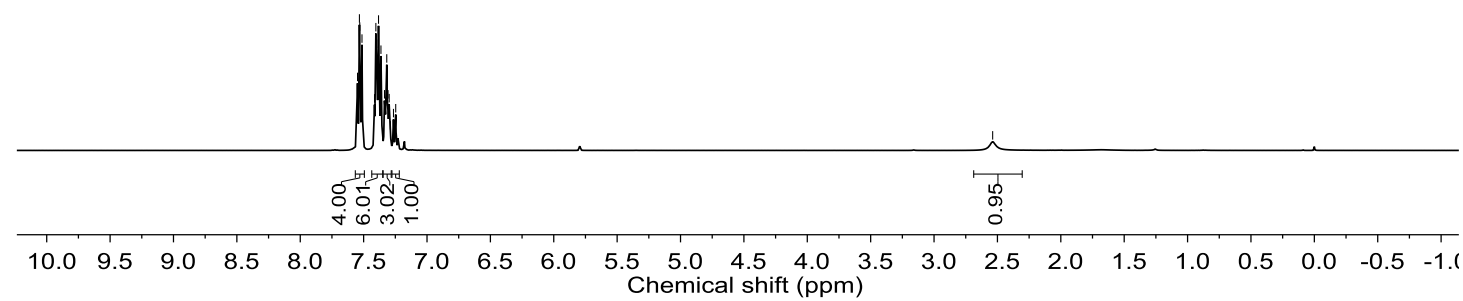


<smiles>[2H]C(O)(c1ccccc1)c1ccc(-c2ccccc2)cc1</smiles>

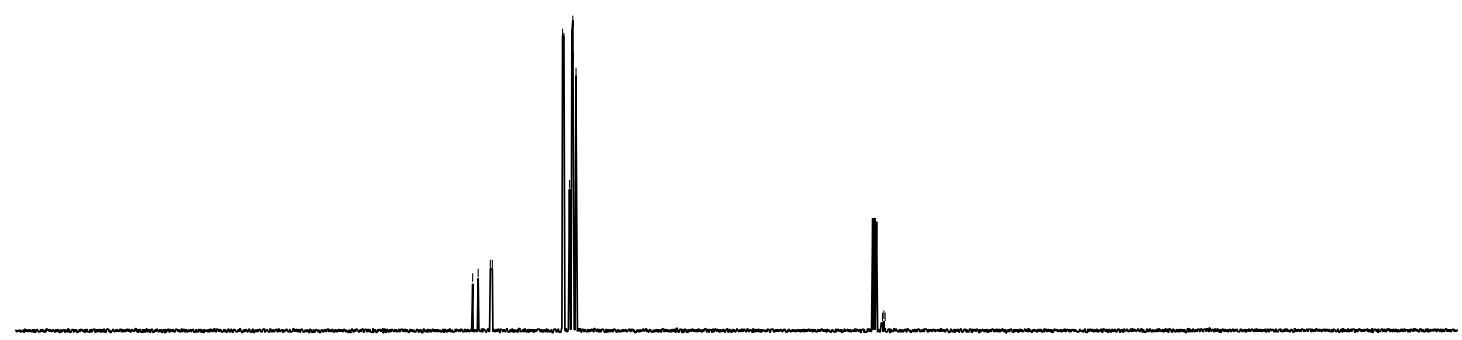

$\begin{array}{llllllllllllllllllllllll}210 & 200 & 190 & 180 & 170 & 160 & 150 & 140 & 130 & 120 & \begin{array}{c}110 \\ \mathrm{f} 1\end{array}(\mathrm{ppm}) & 90 & 80 & 70 & 60 & 50 & 40 & 30 & 20 & 10 & 0 & -10\end{array}$
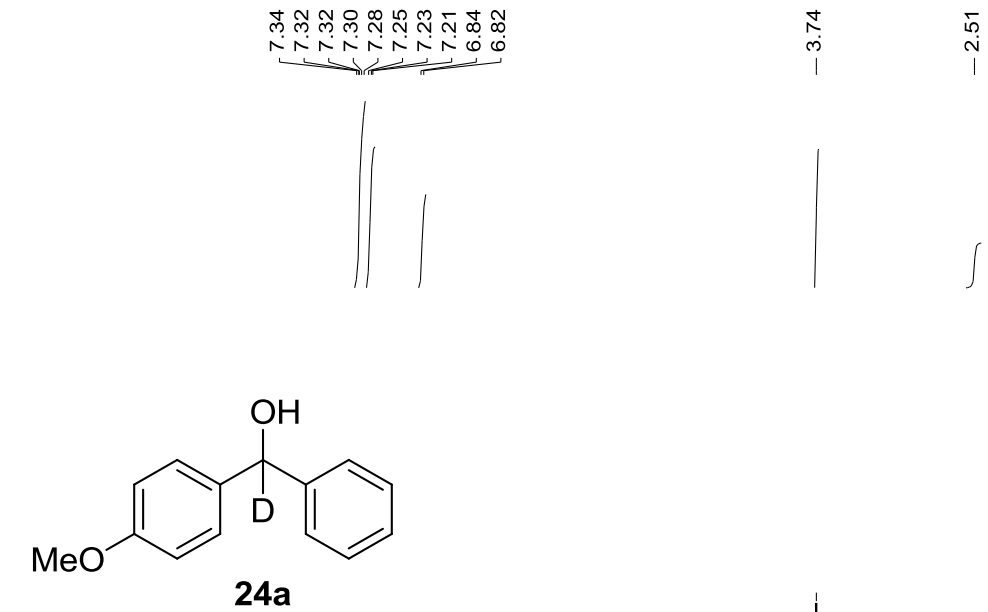

$24 a$

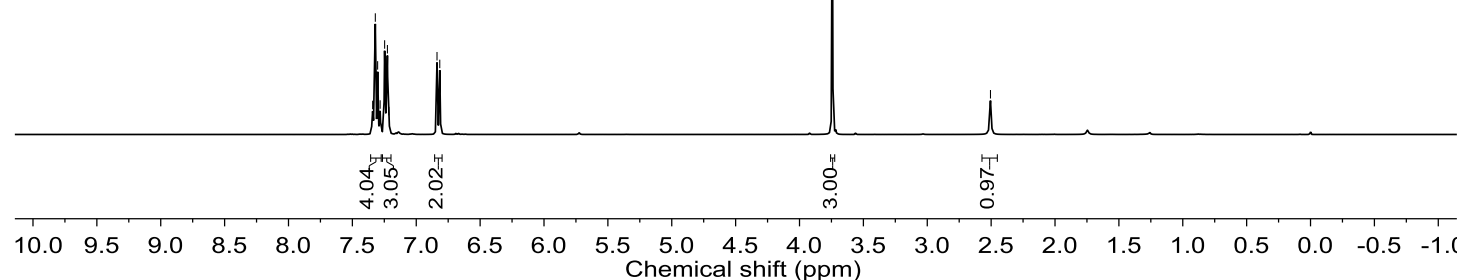


<smiles>[Z10]C([2H])(O)c1ccc(OC)cc1</smiles>

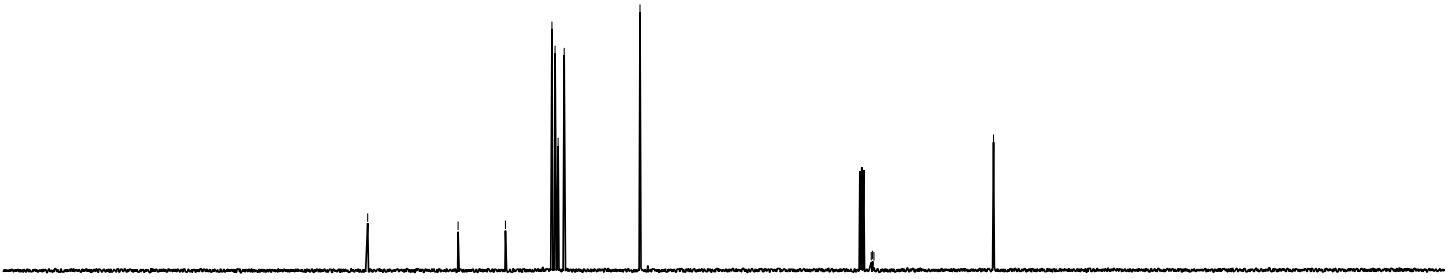

$\begin{array}{lllllllllllllllllllllll}210 & 200 & 190 & 180 & 170 & 160 & 150 & 140 & 130 & 120 & 110 & 100 & 90 & 80 & 70 & 60 & 50 & 40 & 30 & 20 & 10 & 0 & -10\end{array}$ Chemical shift (ppm)
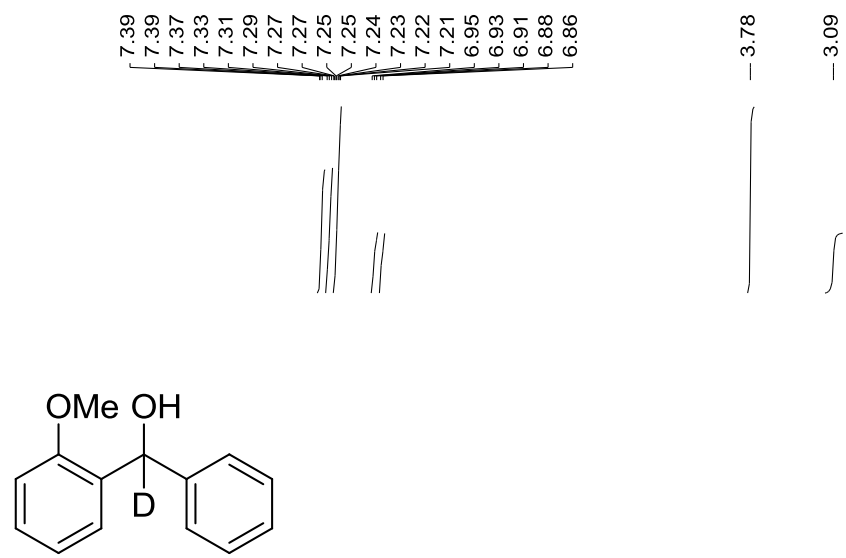

$25 a$

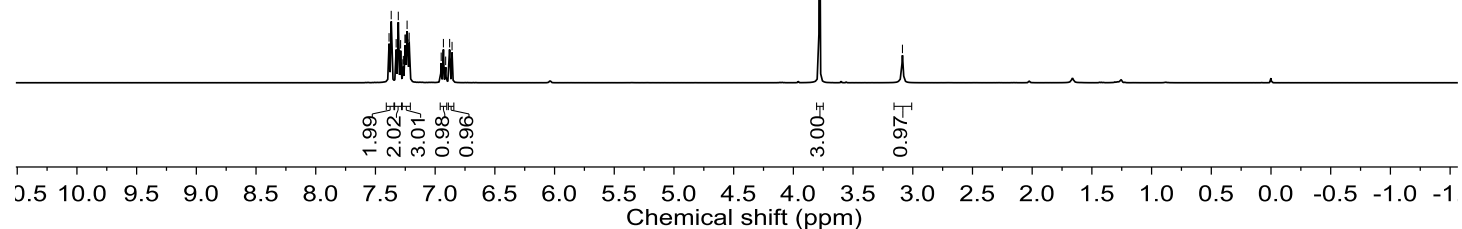


<smiles>COc1ccccc1C(O)(O)c1ccccc1</smiles>

25a

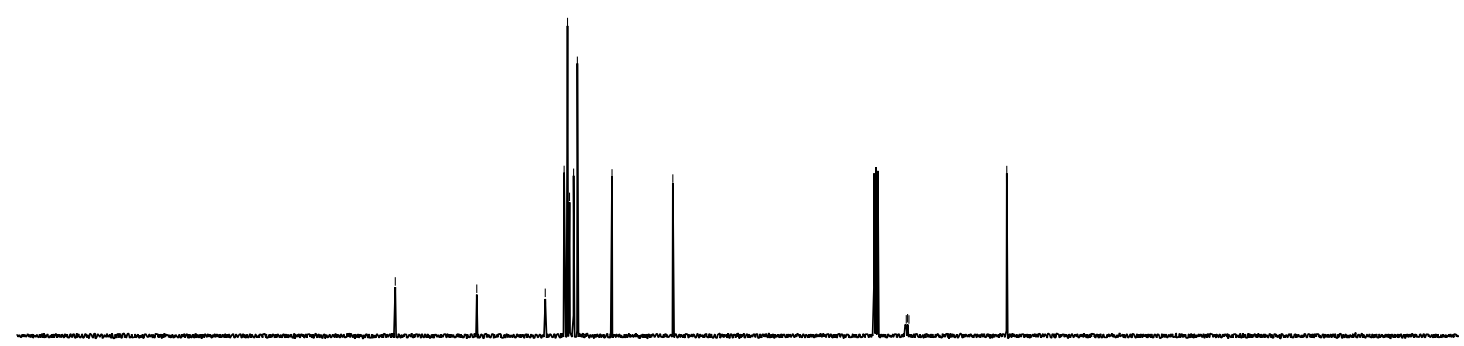

$\begin{array}{llllllllllllllllllllll}210 & 200 & 190 & 180 & 170 & 160 & 150 & 140 & 130 & \begin{array}{c}120 \\ \text { Chemical shift (ppm) }\end{array} 110 & 100 & 90 & 80 & 70 & 60 & 50 & 40 & 30 & 20 & 10 & 0 & -10\end{array}$

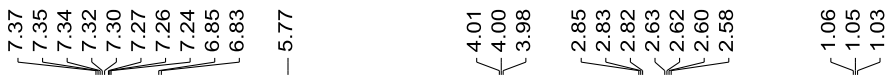<smiles>C1=CCCC=C1</smiles>

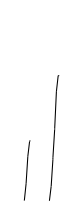<smiles>[R6]C(O)(c1ccccc1)c1ccc(OCCN(CC)CC)cc1</smiles>

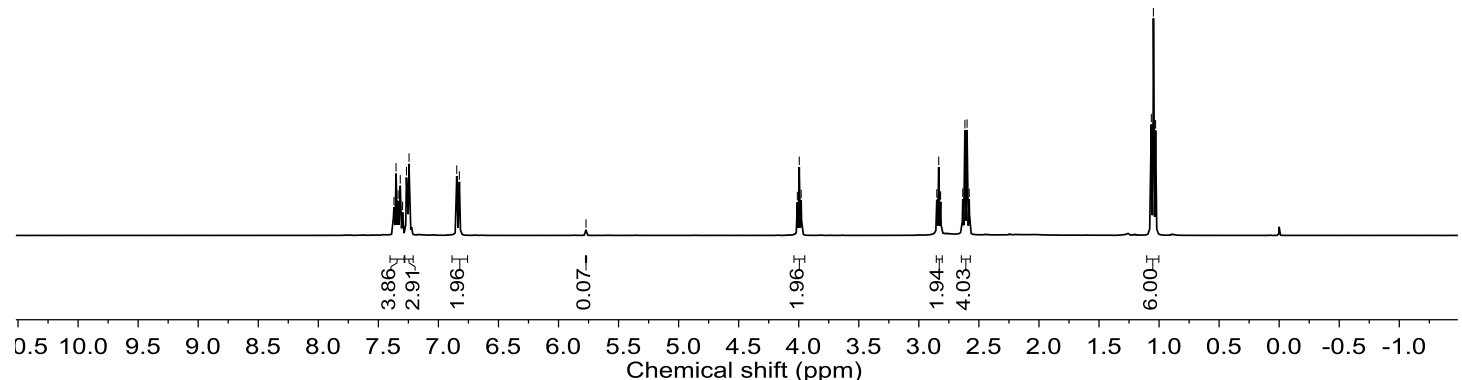


<smiles>[R6]C(O)(c1ccccc1)c1ccc(OCCN(CC)CC)cc1</smiles>

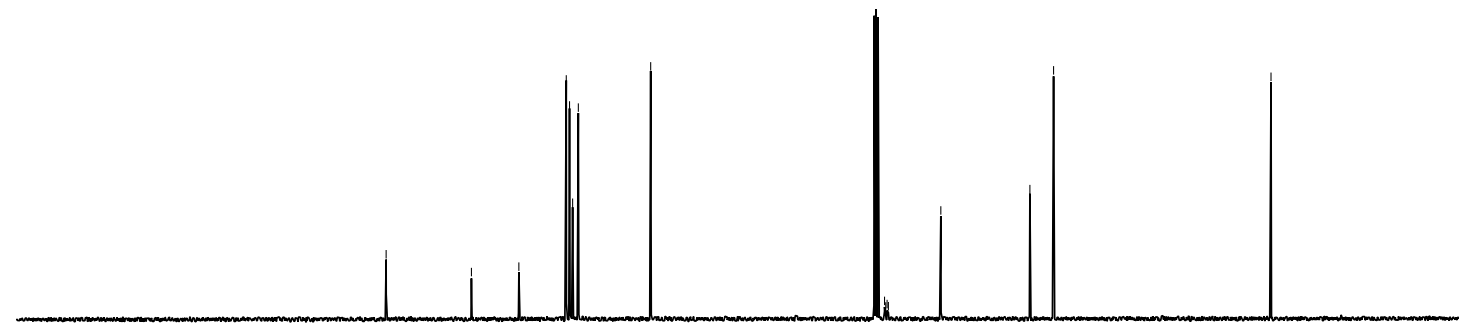

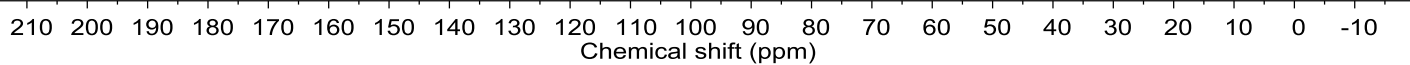

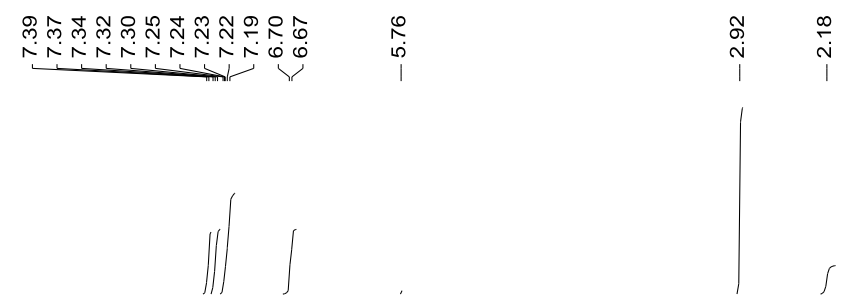<smiles>[R]C(O)(c1ccccc1)c1ccc(N(C)C)cc1</smiles>

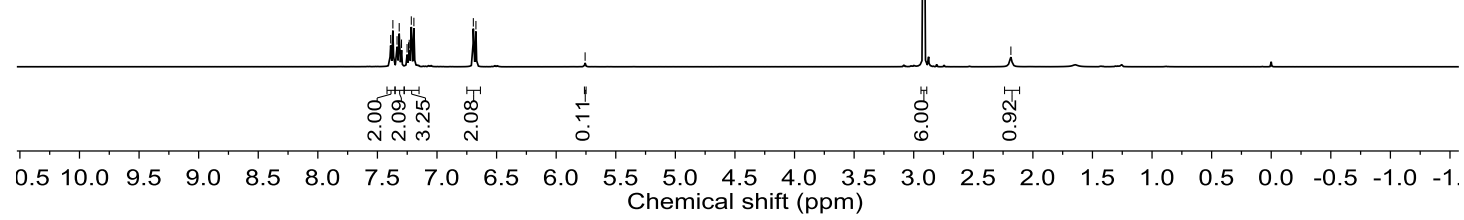


<smiles>[2H]C(O)(c1ccccc1)c1ccc(N(C)C)cc1</smiles>

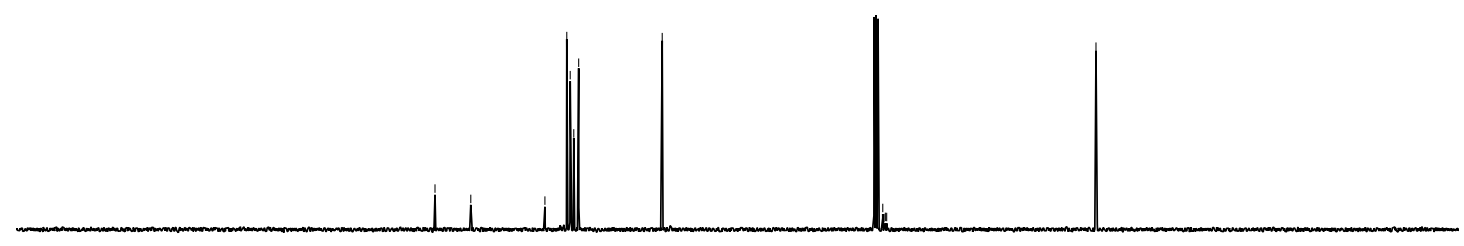

$\begin{array}{llllllllllllllllllllllllll}210 & 200 & 190 & 180 & 170 & 160 & 150 & 140 & 130 & 120 & 110 & 100 & 90 & 80 & 70 & 60 & 50 & 40 & 30 & 20 & 10 & 0 & -10\end{array}$

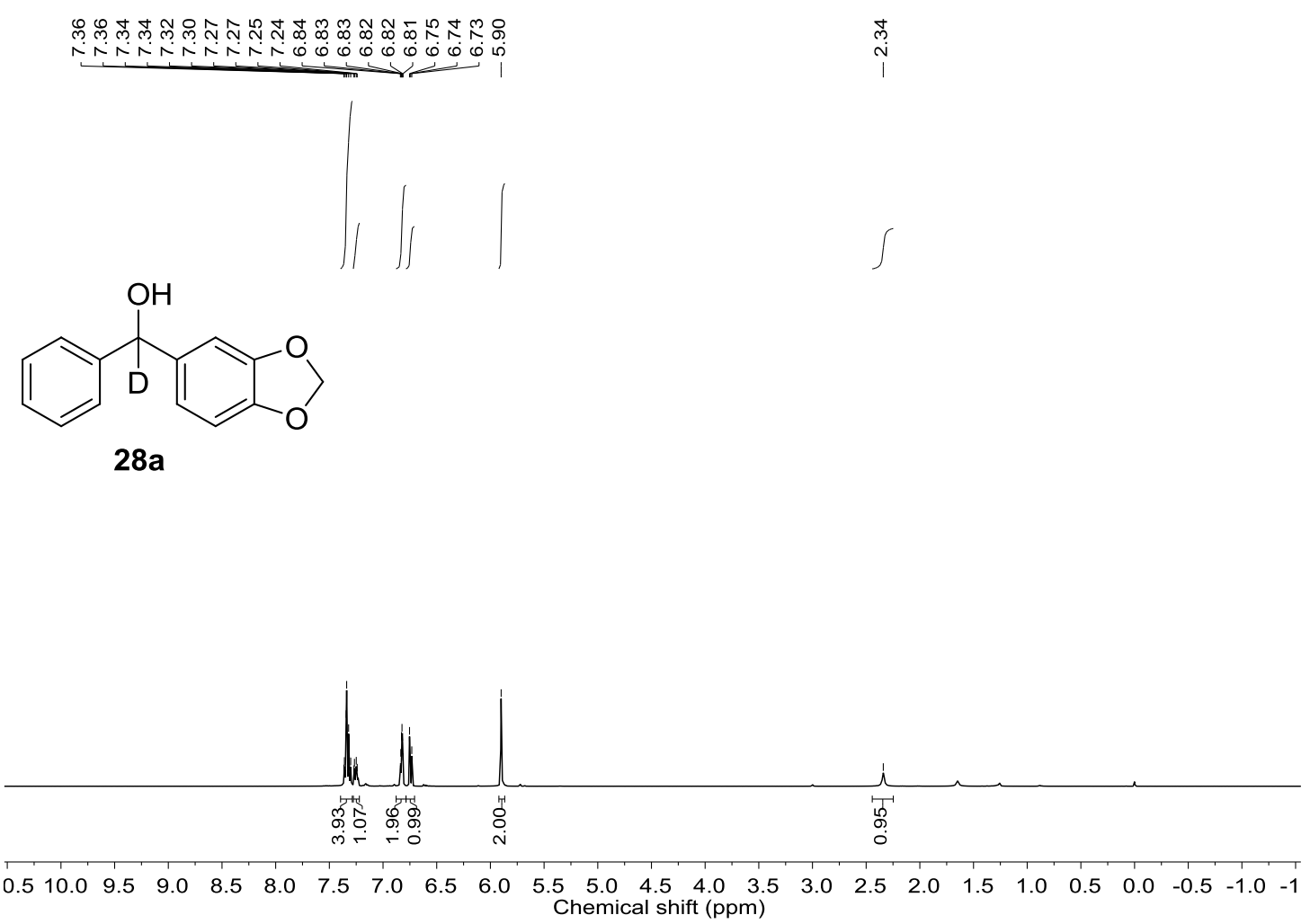


<smiles>[2H]C(O)(c1ccccc1)c1ccc2c(c1)OCO2</smiles>

$28 \mathrm{a}$

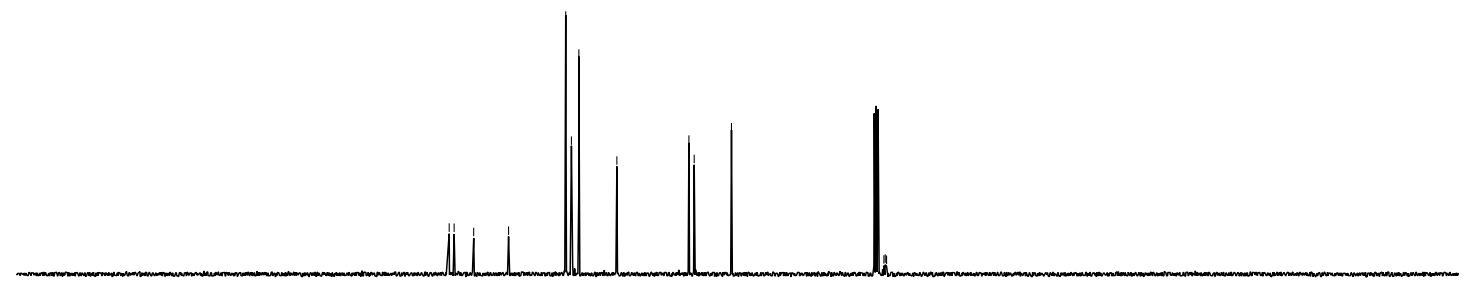
$\begin{array}{lllllllllllllllllllll}210 & 200 & 190 & 180 & 170 & 160 & 150 & 140 & 130 & \begin{array}{c}120 \\ \text { Chemical shift (ppm) }\end{array} & 110 & 100 & 90 & 80 & 50 & 40 & 30 & 20 & 10 & 0 & -10\end{array}$

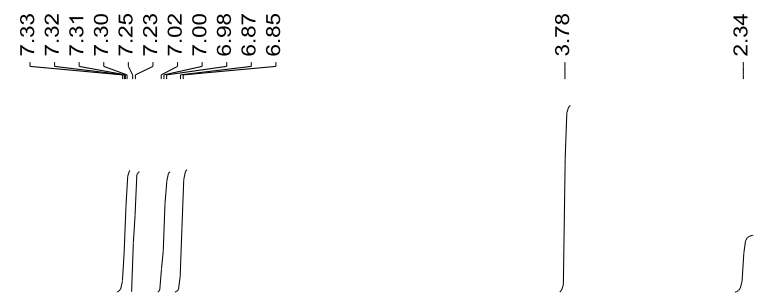<smiles>[2H]C(O)(c1ccc(F)cc1)c1ccc(OC)cc1</smiles>

29a

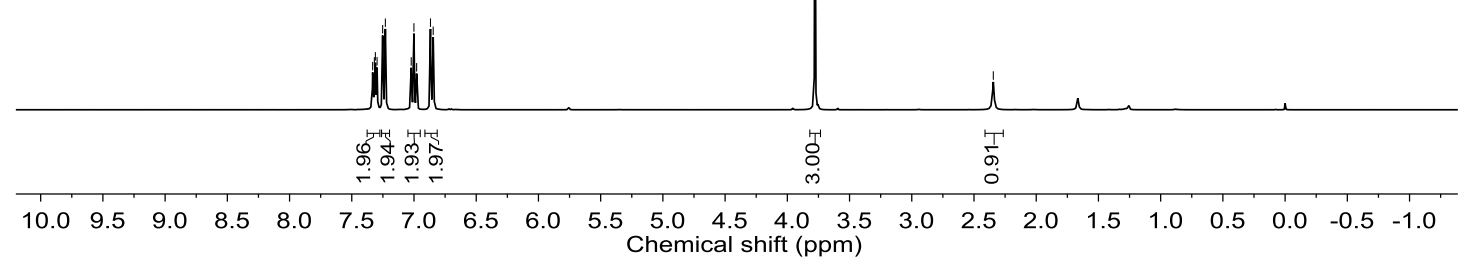



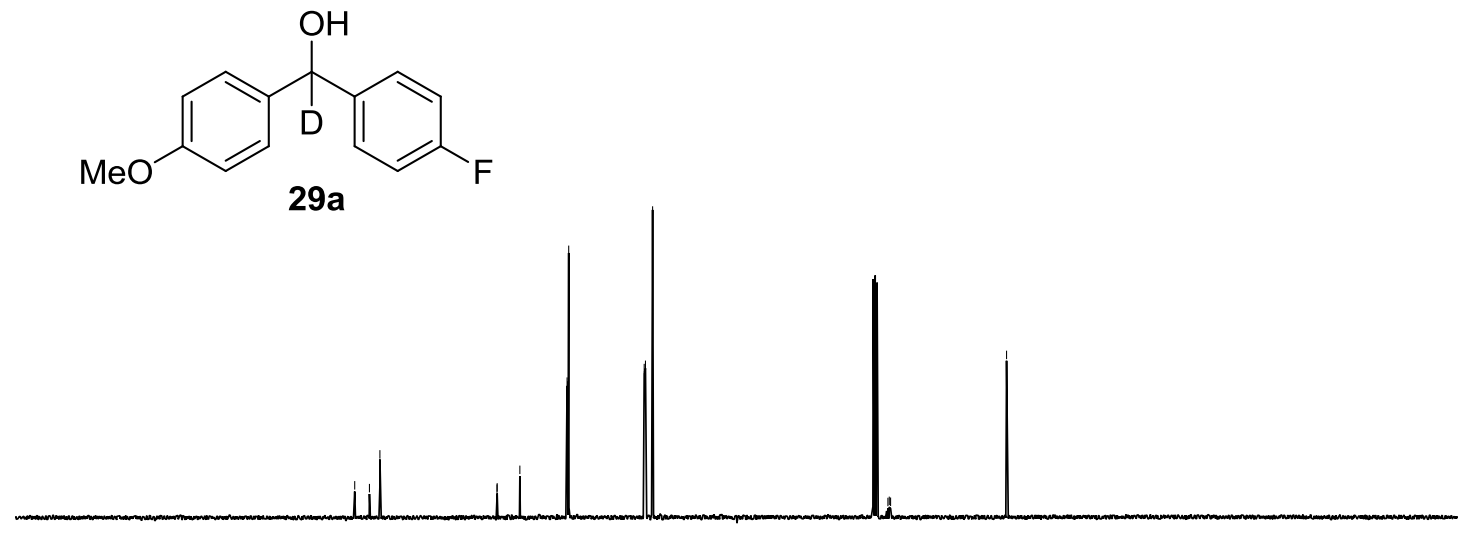

$\begin{array}{lllllllllllllllllllllll}210 & 200 & 190 & 180 & 170 & 160 & 150 & 140 & 130 & 120 & 110 & 100 & 90 & 80 & 70 & 60 & 50 & 40 & 30 & 20 & 10 & 0 & -10\end{array}$ Chemical shift (ppm)<smiles>COc1ccc(C(O)([18OH])c2ccc(F)cc2)cc1</smiles>

29a

$\begin{array}{lllllllllllllllllllllllll}10 & 0 & -10 & -20 & -30 & -40 & -50 & -60 & -70 & -80 & -90 & -100 & -110 & -120 & -130 & -140 & -150 & -160 & -170 & -180 & -190 & -200 & -210\end{array}$ Chemical shift (ppm) 


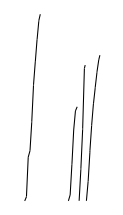<smiles>[2H]C(O)(c1ccccc1)c1ccc2ccccc2c1</smiles>

$30 a$
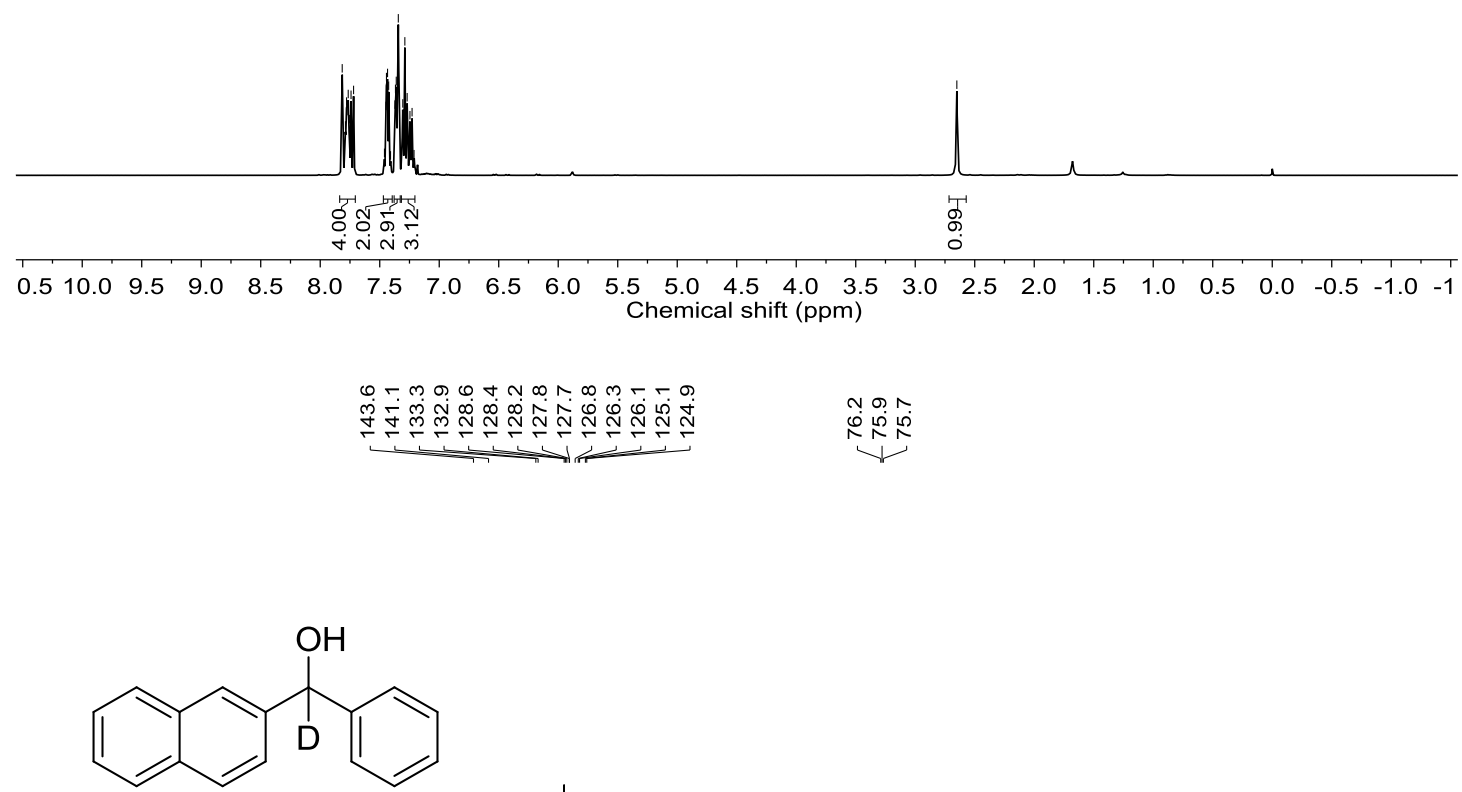

$30 a$

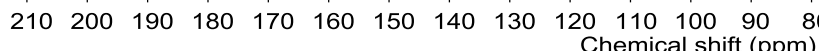




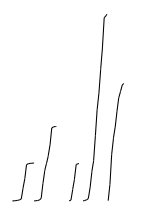<smiles>[2H]C(O)(c1ccccc1)c1cccc2ccccc12</smiles>

$31 a$

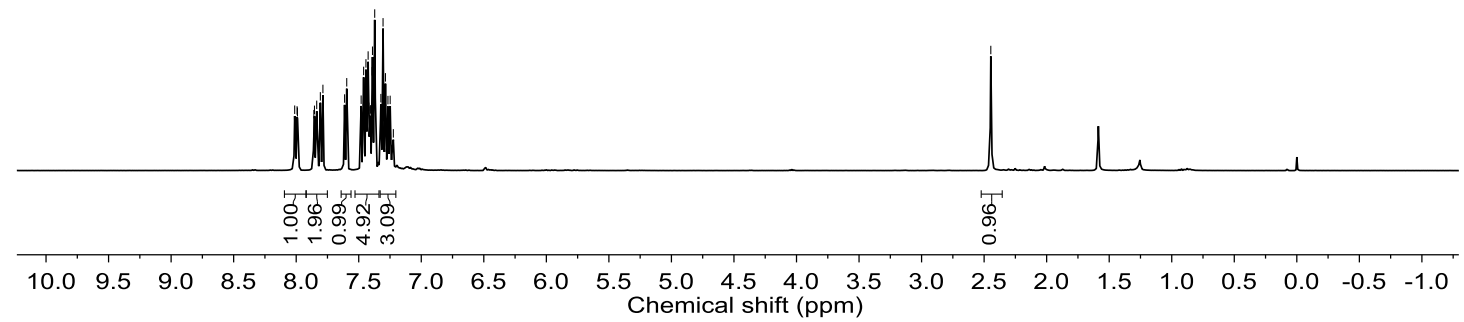

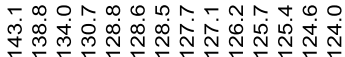

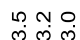

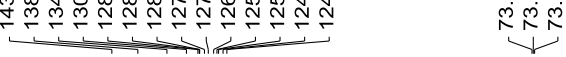<smiles>[2H]C(O)(c1ccccc1)c1cccc2ccccc12</smiles>

$31 a$

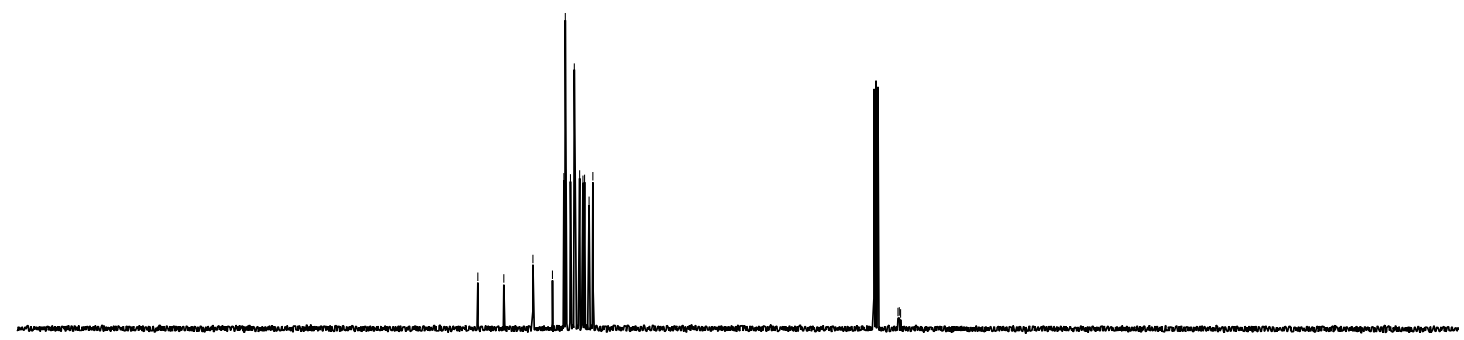

$\begin{array}{lllllllllllllllllllllllll}210 & 200 & 190 & 180 & 170 & 160 & 150 & 140 & 130 & 120 & 110 & 100 & 90 & 80 & 70 & 60 & 50 & 40 & 30 & 20 & 10 & 0 & -10\end{array}$ Chemical shift (ppm) 


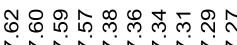

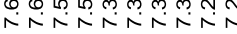
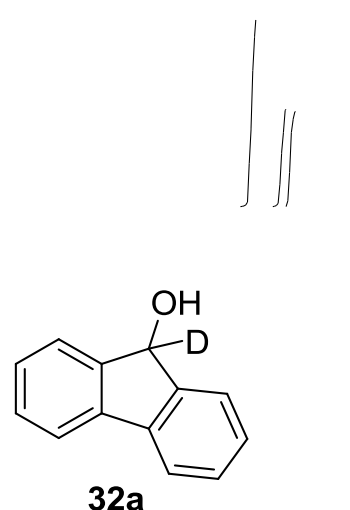

$32 a$

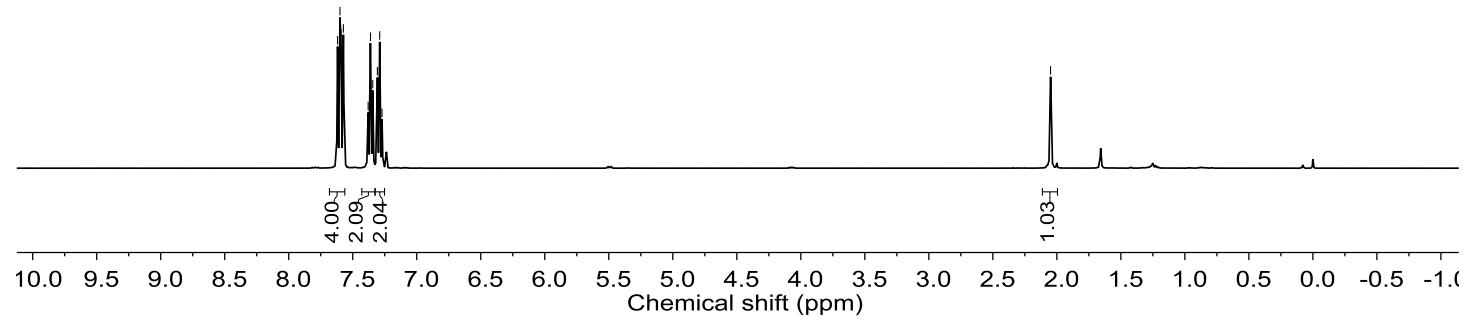

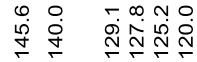

官宾交

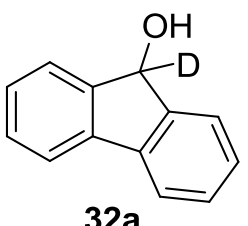

$32 a$

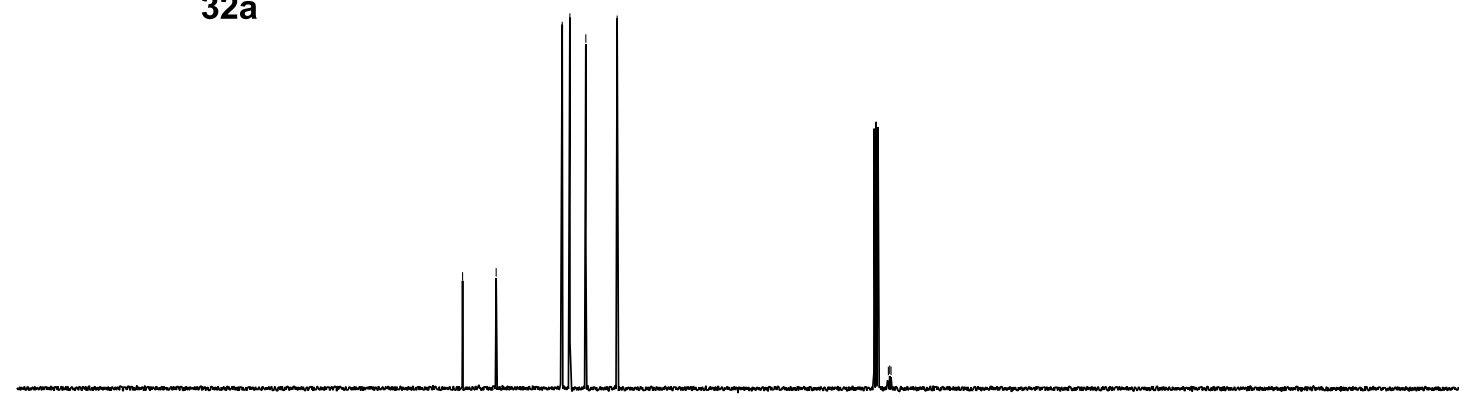

$\begin{array}{lllllllllllllllllllllll}210 & 200 & 190 & 180 & 170 & 160 & 150 & 140 & 130 & 120 & 110 & 100 & 90 & 80 & 70 & 60 & 50 & 40 & 30 & 20 & 10 & 0 & -10\end{array}$ Chemical shift (ppm) 


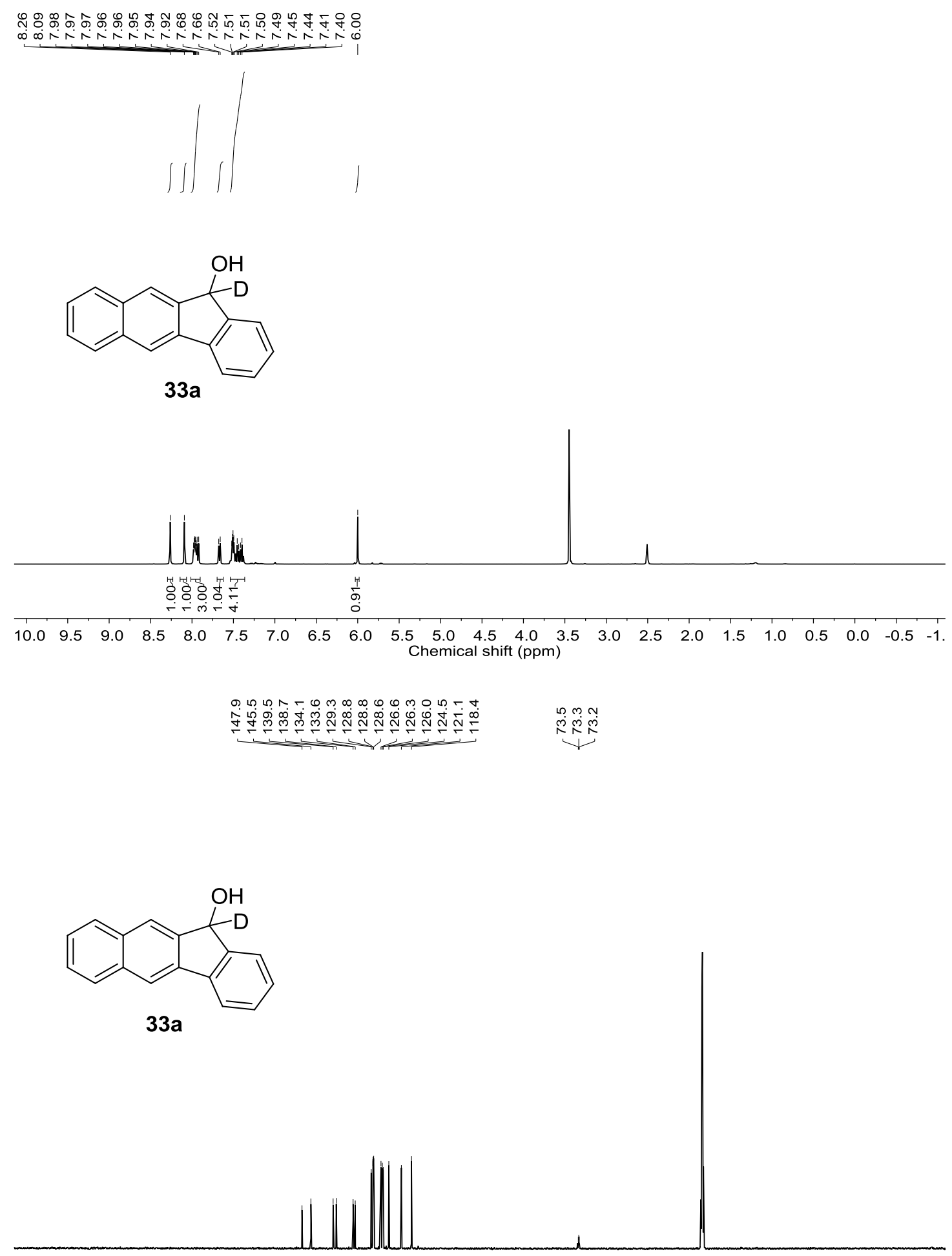

$\begin{array}{llllllllllllllllllllllllllllllll}220 & 210 & 200 & 190 & 180 & 170 & 160 & 150 & 140 & 130 & 120 & 110 & 100 & 90 & 80 & 70 & 60 & 50 & 40 & 30 & 20 & 10 & 0 & -10 & -20\end{array}$ Chemical shift (ppm) 

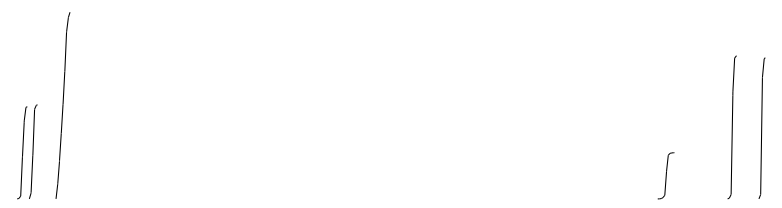<smiles></smiles>

$34 a$

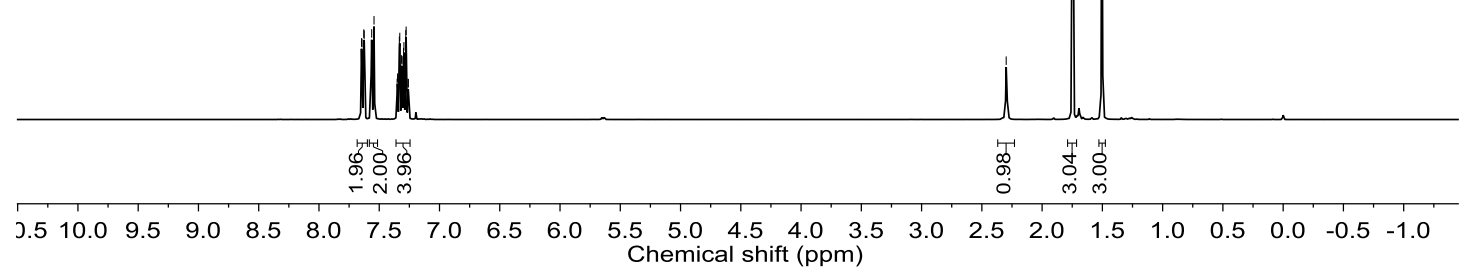

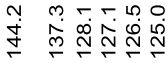

$\underbrace{090}$<smiles>CC1(C)c2ccccc2C2(O)CC1(C)c1ccccc12</smiles>

$34 a$

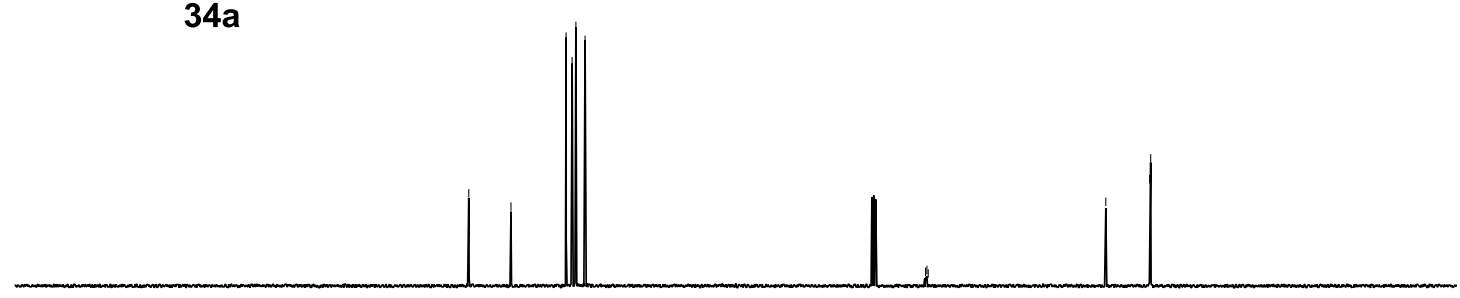

$\begin{array}{llllllllllllllllllllllll}210 & 200 & 190 & 180 & 170 & 160 & 150 & 140 & 130 & 120 & 110 & 100 & 90 & 80 & 70 & 60 & 50 & 40 & 30 & 20 & 10 & 0 & -10\end{array}$ Chemical shift (ppm) 


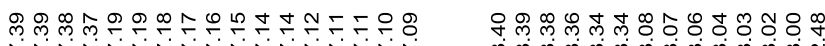

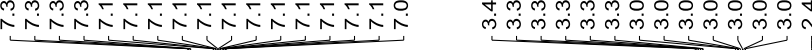

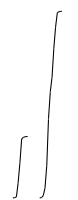<smiles>[2H]C1(O)c2ccccc2CCc2ccccc21</smiles>

$35 a$

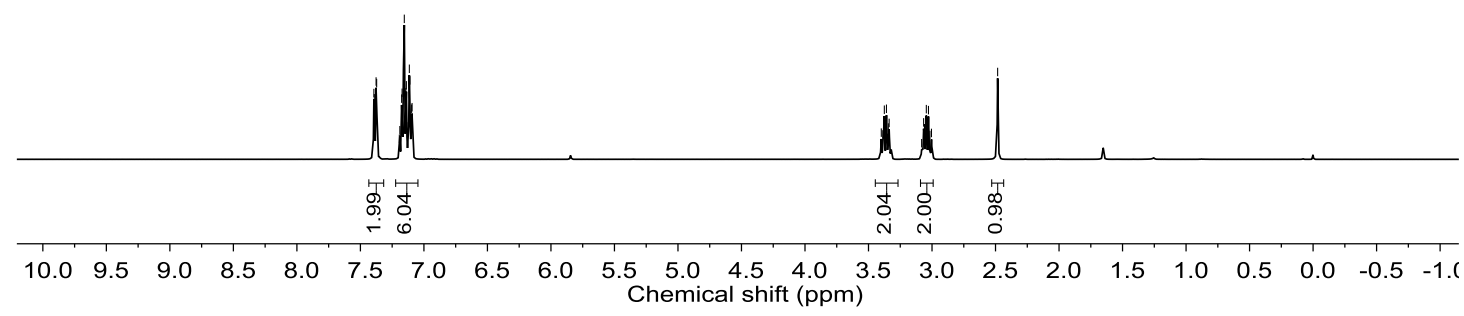

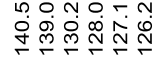

i)
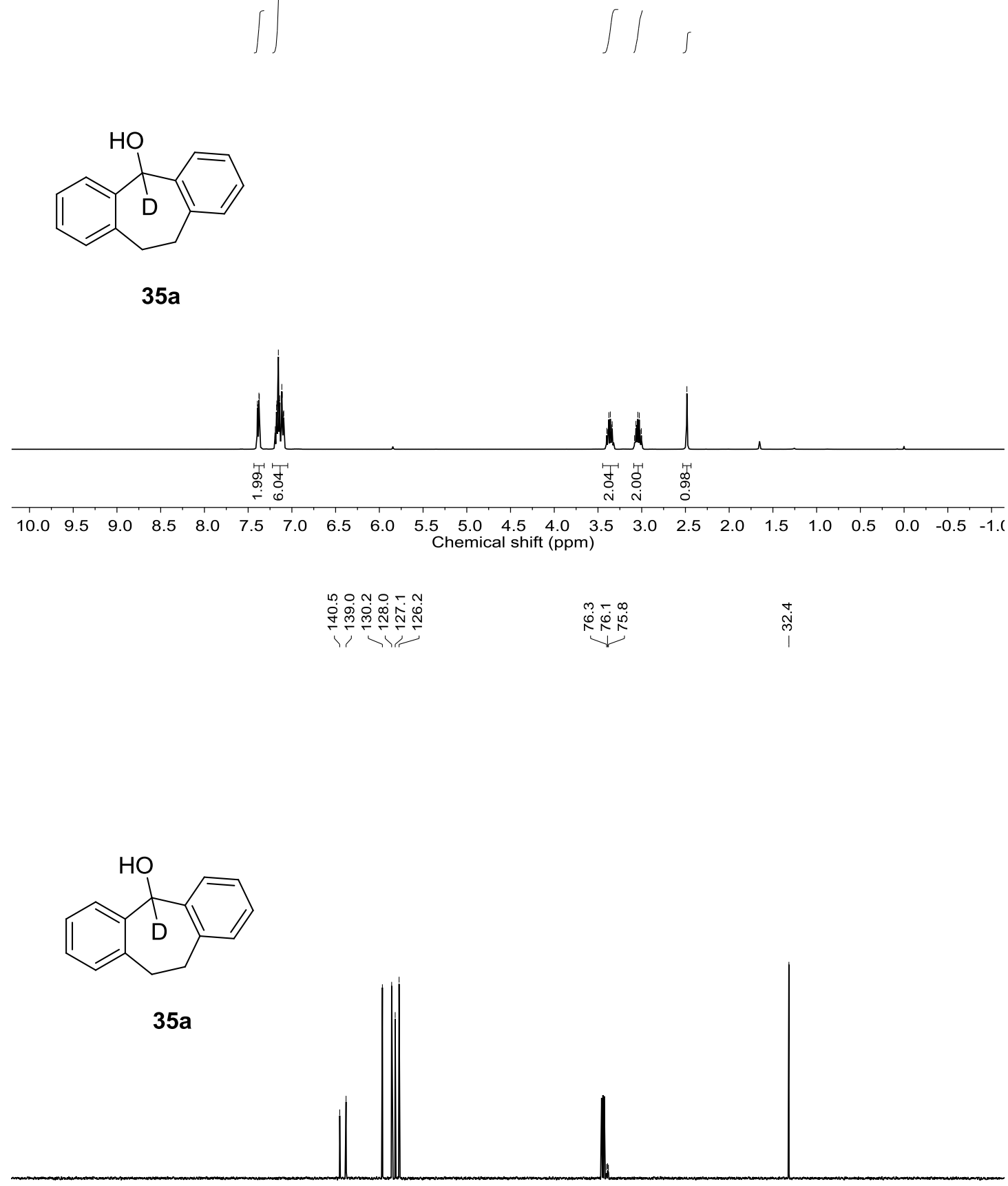

$\begin{array}{llllllllllllllllllllllll}210 & 200 & 190 & 180 & 170 & 160 & 150 & 140 & 130 & 120 & 110 & 100 & 90 & 80 & 70 & 60 & 50 & 40 & 30 & 20 & 10 & 0 & -10\end{array}$ Chemical shift (ppm) 


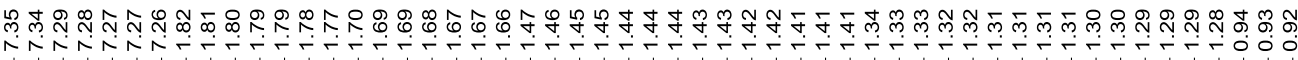

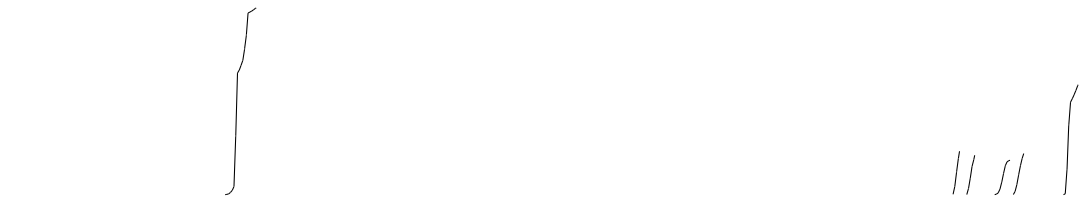<smiles>[2H]C(O)(CCC)c1ccccc1</smiles>

$36 a$

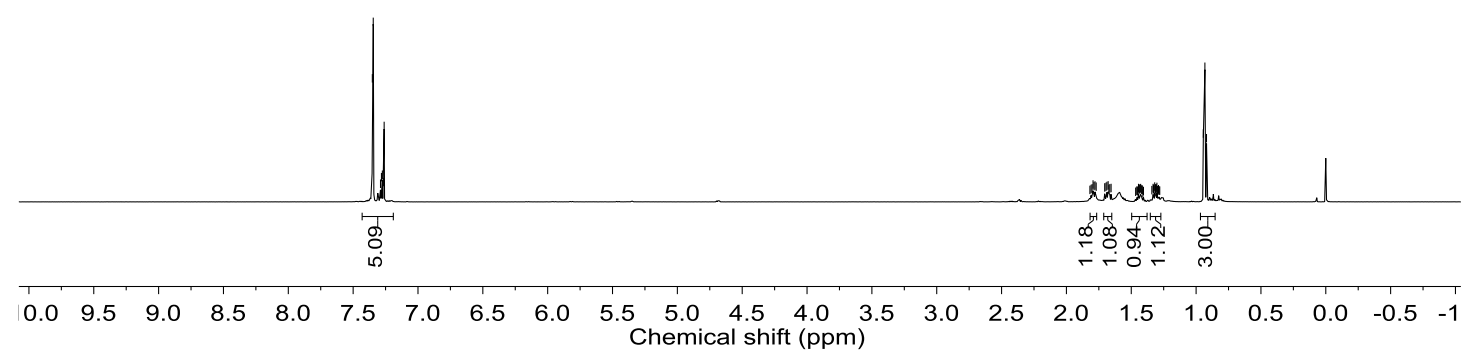

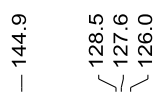

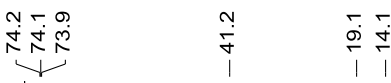<smiles>[2H]C(O)(CCC)c1ccccc1</smiles>

$36 a$

$\begin{array}{llllllllllllllllllllllllllllllllll}220 & 210 & 200 & 190 & 180 & 170 & 160 & 150 & 140 & 130 & 120 & 110 & 100 & 90 & 80 & 70 & 60 & 50 & 40 & 30 & 20 & 10 & 0 & -10 & -20\end{array}$ Chemical shift (ppm) 
<smiles>CCCCCCC</smiles><smiles>[2H]C(O)(c1ccccc1)c1ccccn1</smiles>

$38 a$

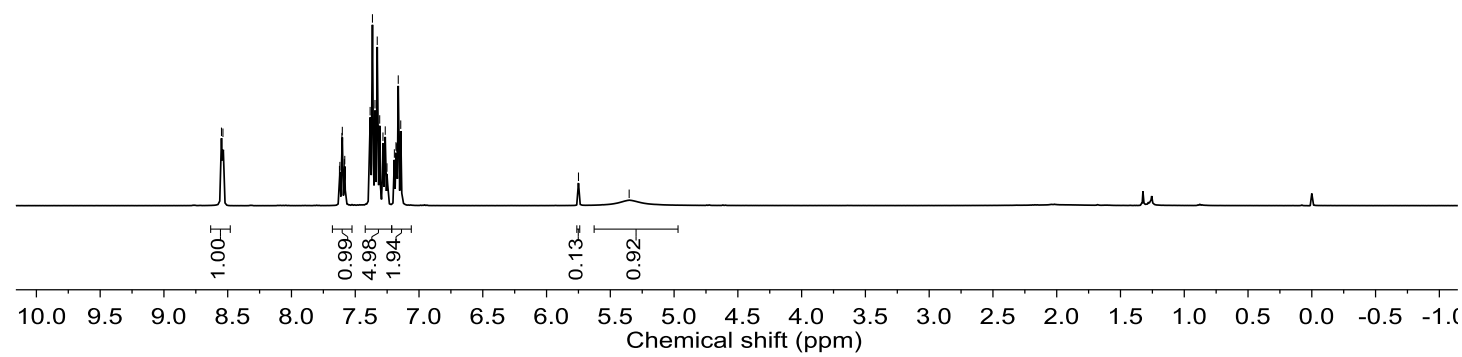

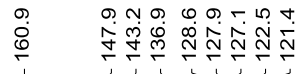

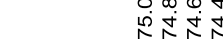

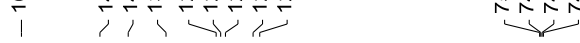

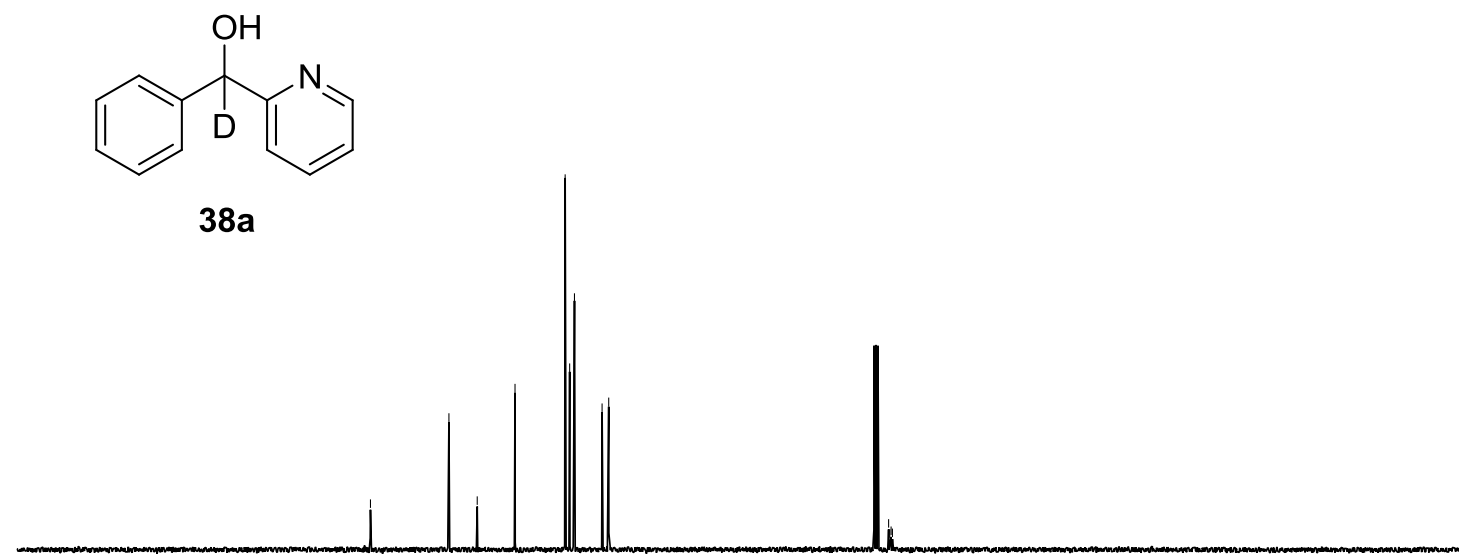

$\begin{array}{lllllllllllllllllllllllll}210 & 200 & 190 & 180 & 170 & 160 & 150 & 140 & 130 & 120 & 110 & 100 & 90 & 80 & 70 & 60 & 50 & 40 & 30 & 20 & 10 & 0 & -10\end{array}$ Chemical shift (ppm) 


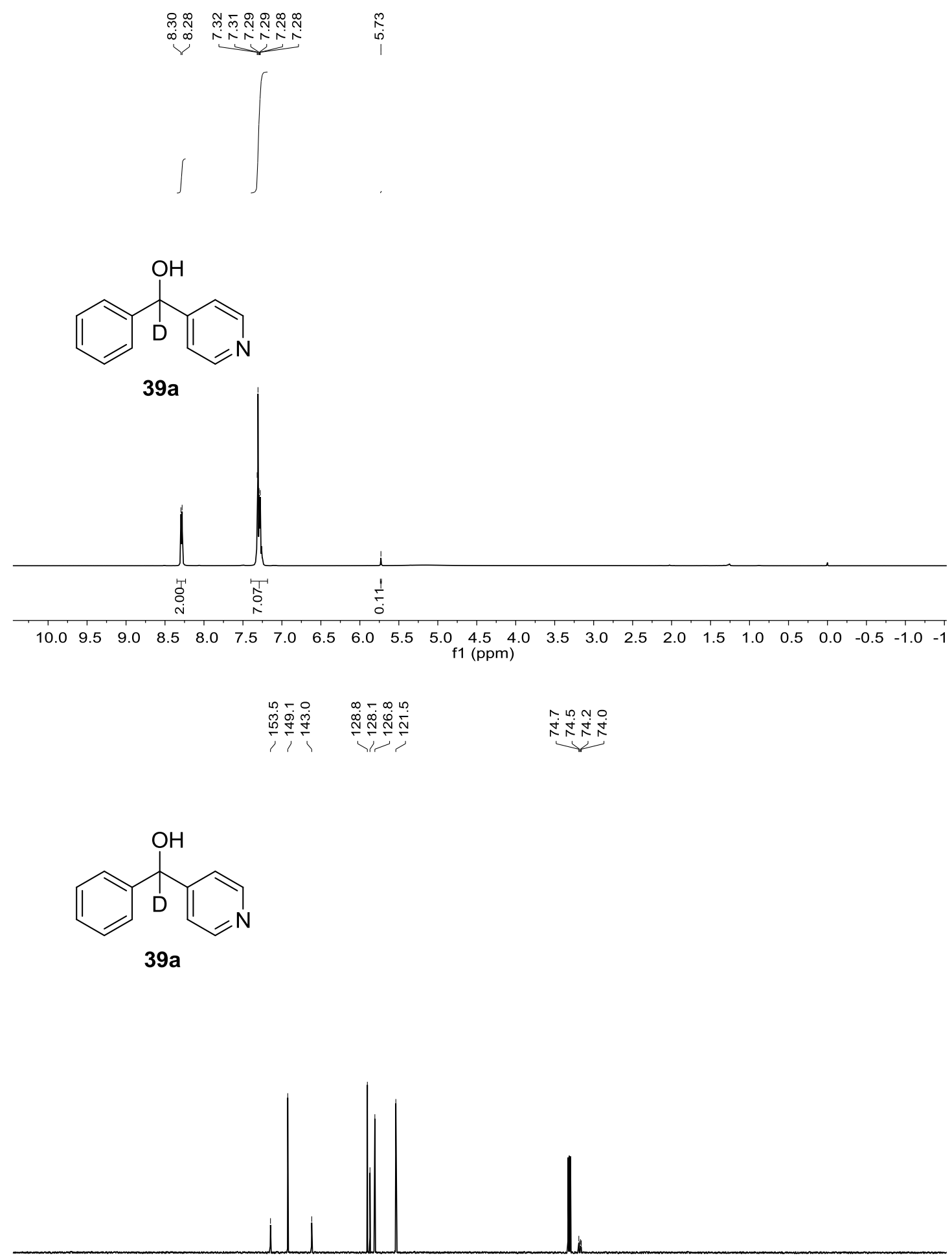

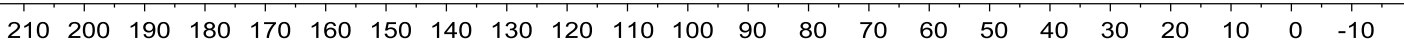
Chemical shift (ppm) 


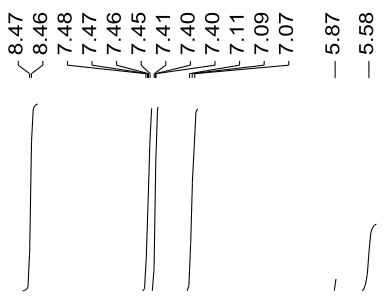<smiles>[2H]C(O)(c1ccncc1)c1ccc(F)cc1</smiles>

$40 a$
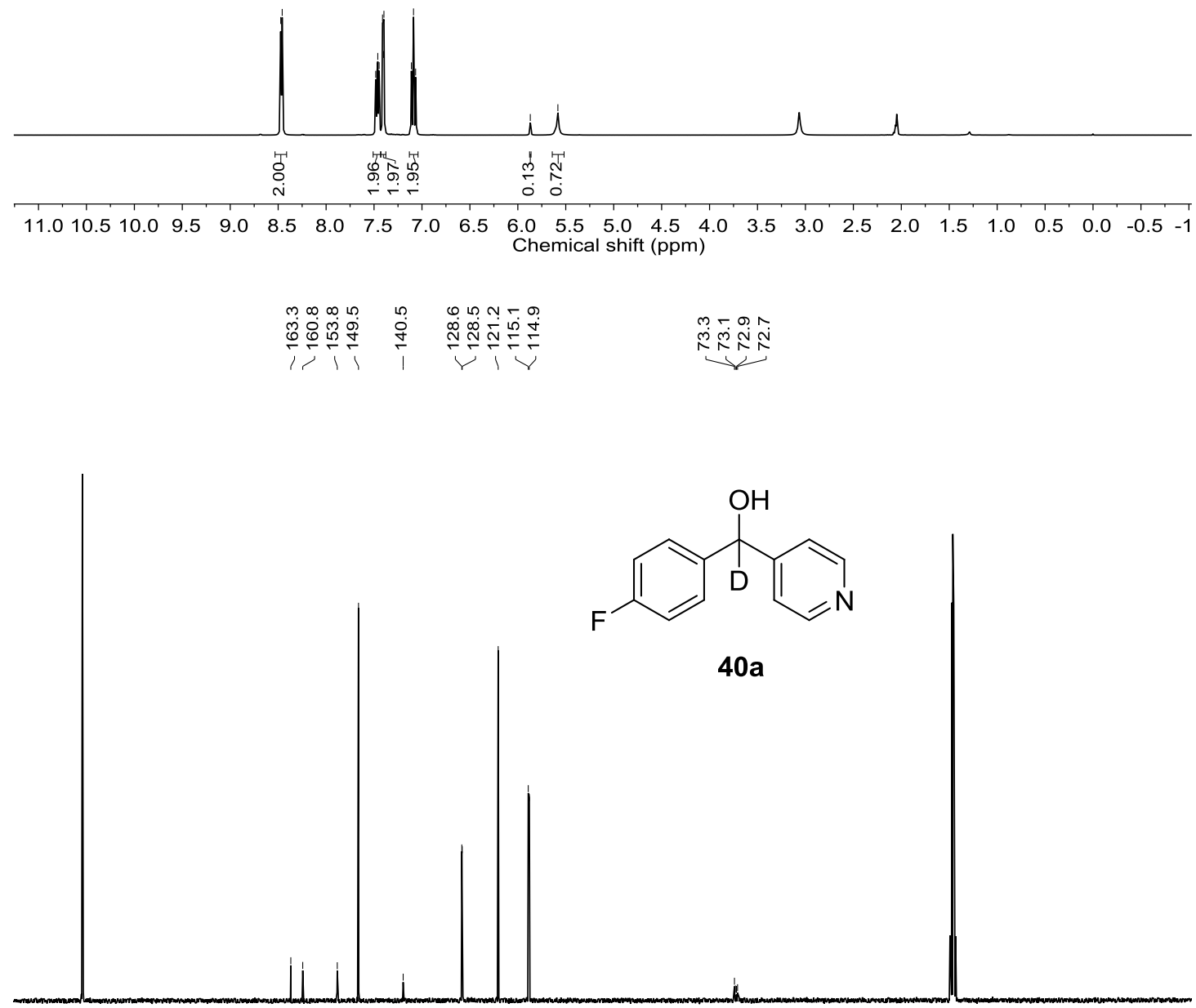

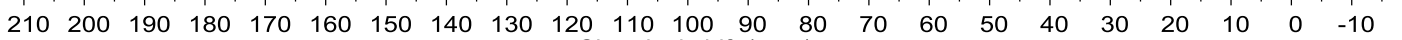
Chemical shift (ppm) 
<smiles>[2H]C(O)(c1ccncc1)c1ccc(F)cc1</smiles>

$40 a$

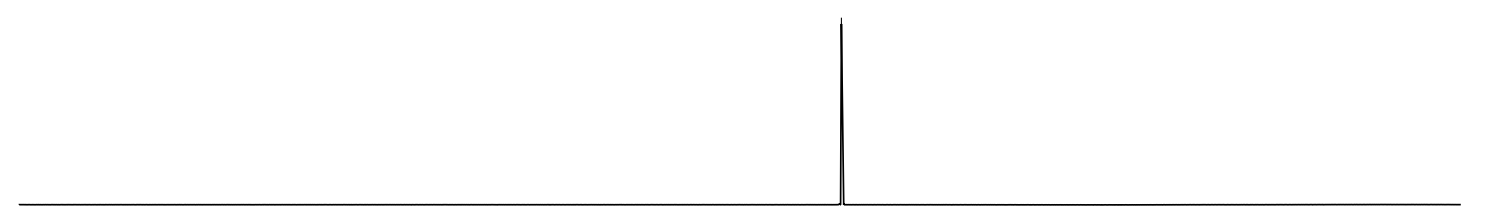

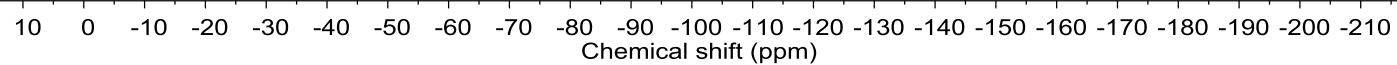

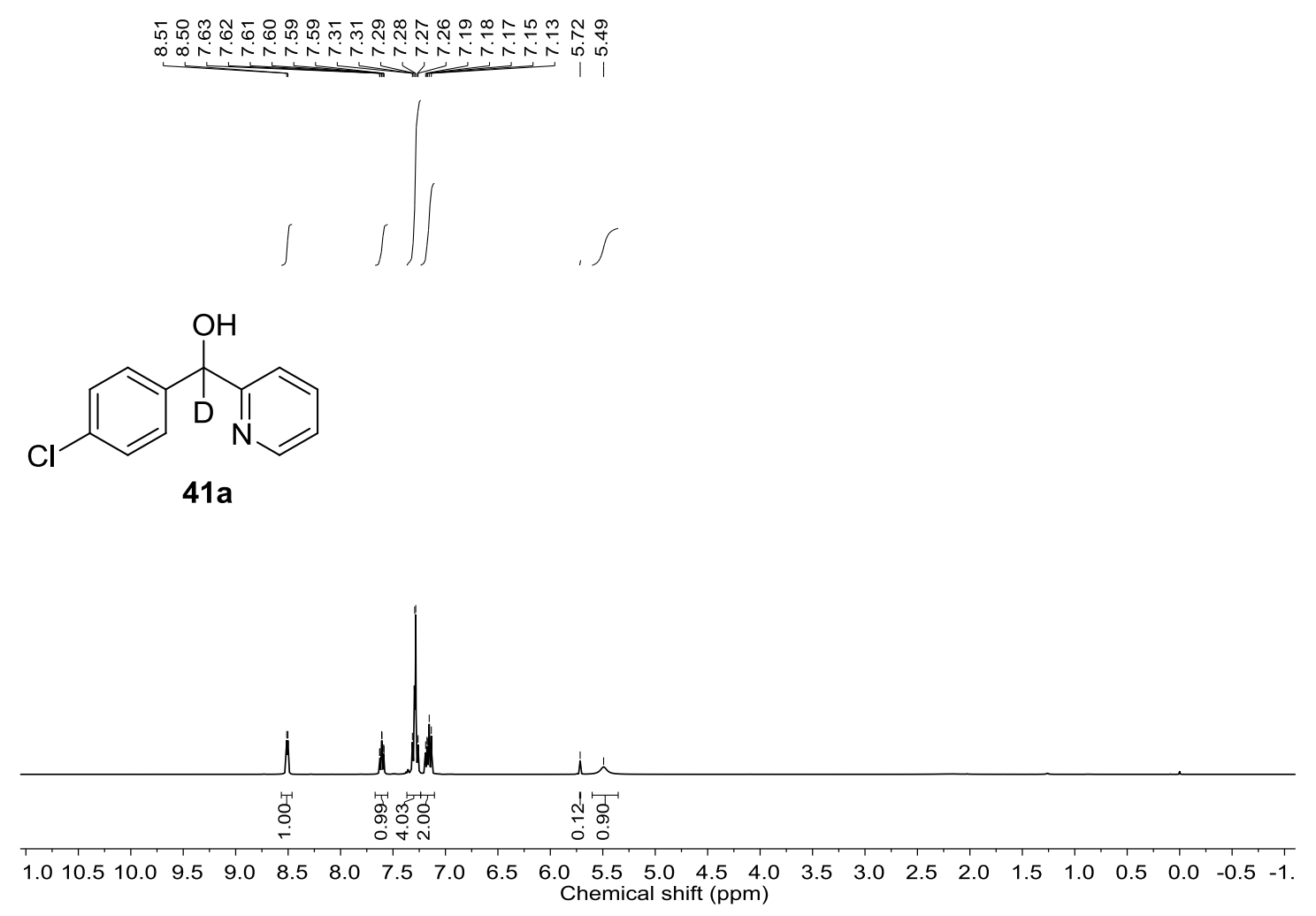


<smiles>OC(O)(c1ccc(Cl)cc1)c1ccccn1</smiles>

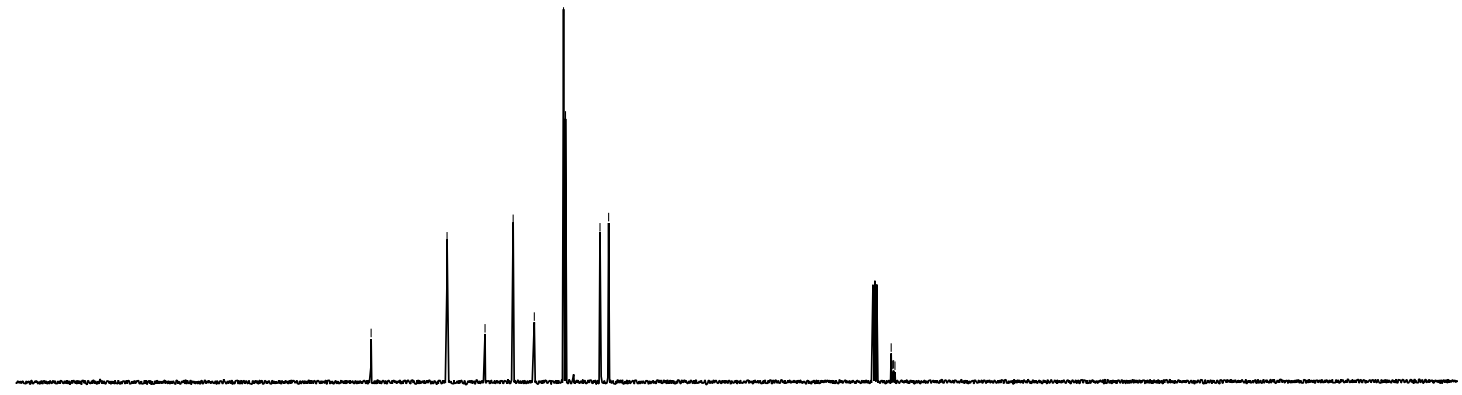

$\begin{array}{lllllllllllllllllllllll}210 & 200 & 190 & 180 & 170 & 160 & 150 & 140 & 130 & 120 & 110 & 100 & 90 & 80 & 70 & 60 & 50 & 40 & 30 & 20 & 10 & 0 & -10\end{array}$ Chemical shift (ppm)

ํํำ \&

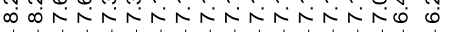
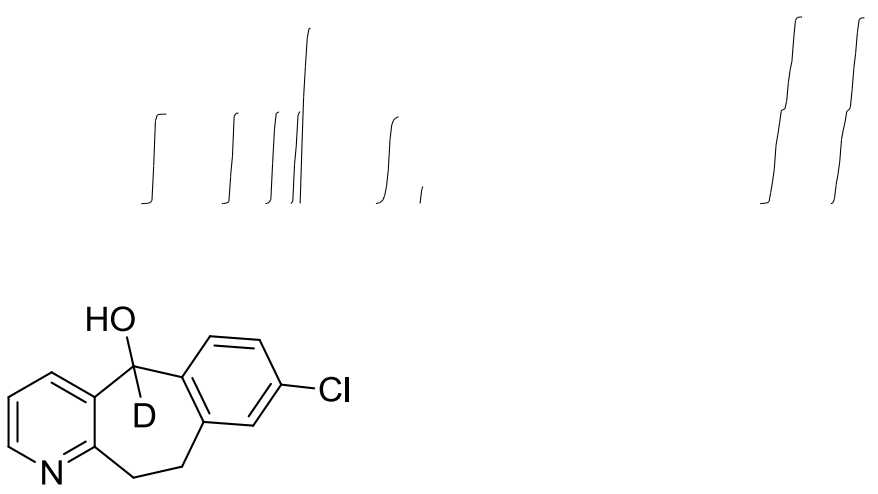

42a

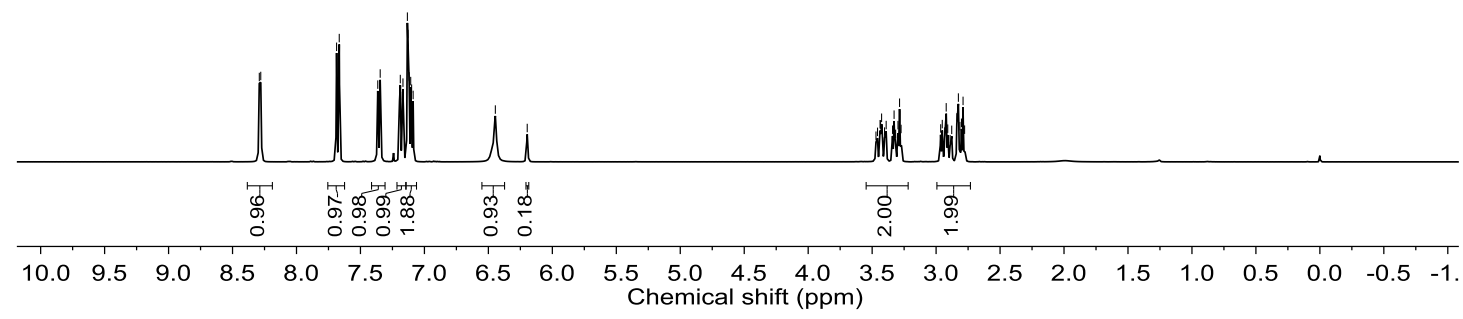

S-73 


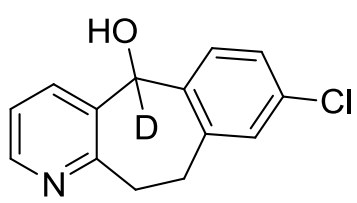

$42 a$

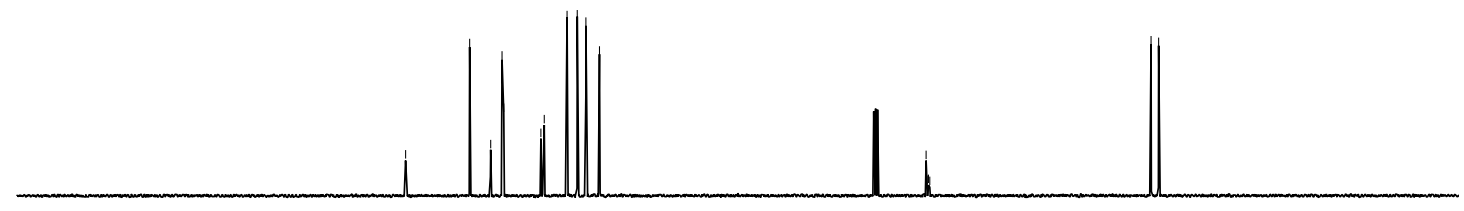

$\begin{array}{lllllllllllllllllllllll}210 & 200 & 190 & 180 & 170 & 160 & 150 & 140 & 130 & 120 & 110 & 100 & 90 & 80 & 70 & 60 & 50 & 40 & 30 & 20 & 10 & 0 & -10\end{array}$ Chemical shift (ppm)

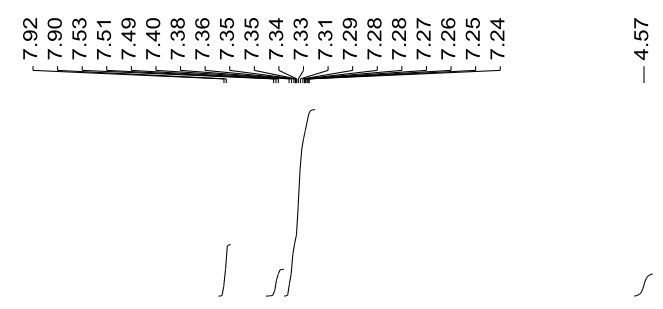<smiles>[2H]C(O)(C(=O)c1ccccc1)c1ccccc1</smiles>

$43 a$

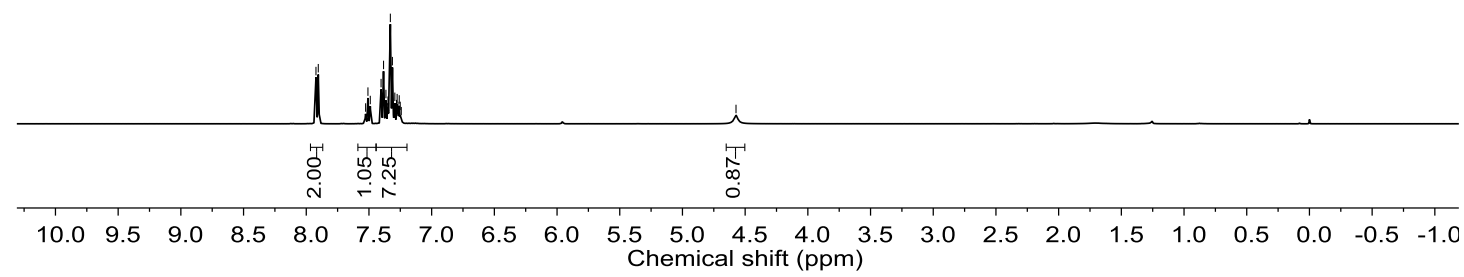


<smiles>[2H]C(O)(C(=O)c1ccccc1)c1ccccc1</smiles>

$43 a$

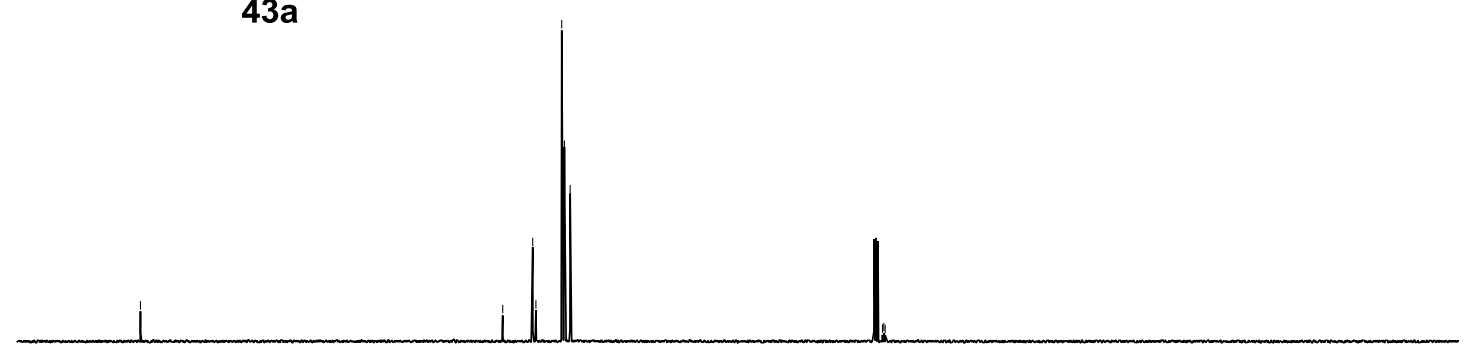

$\begin{array}{lllllllllllllllllllllll}210 & 200 & 190 & 180 & 170 & 160 & 150 & 140 & 130 & 120 & 110 & 100 & 90 & 80 & 70 & 60 & 50 & 40 & 30 & 20 & 10 & 0 & -10\end{array}$ Chemical shift (ppm)

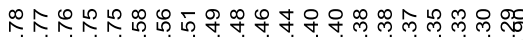

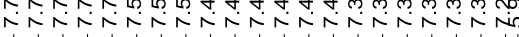

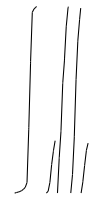<smiles>[2H]C(O)(c1ccccc1)c1ccc(C(=O)c2ccccc2)cc1</smiles>
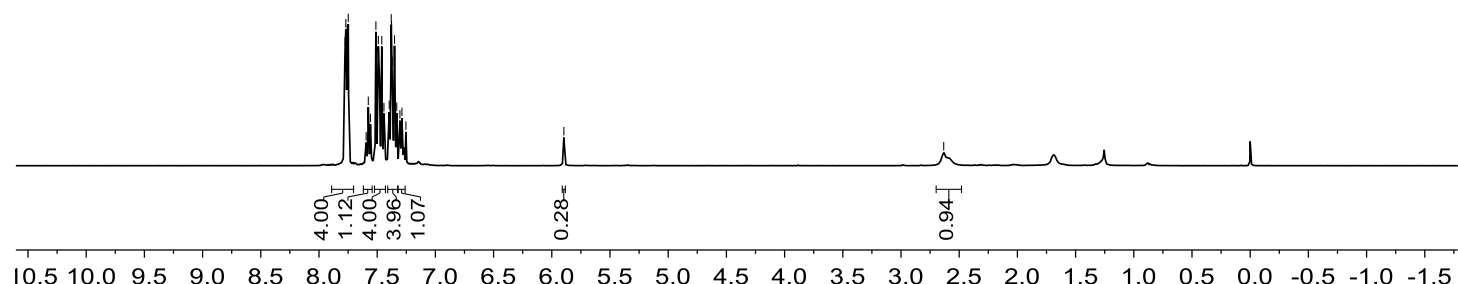

$\begin{array}{lllllllllllllllllllllllll}10.5 & 10.0 & 9.5 & 9.0 & 8.5 & 8.0 & 7.5 & 7.0 & 6.5 & 6.0 & 5.5 & 5.0 & 4.5 & 4.0 & 3.5 & 3.0 & 2.5 & 2.0 & 1.5 & 1.0 & 0.5 & 0.0 & -0.5 & -1.0 & -1.5\end{array}$ Chemical shift (ppm) 


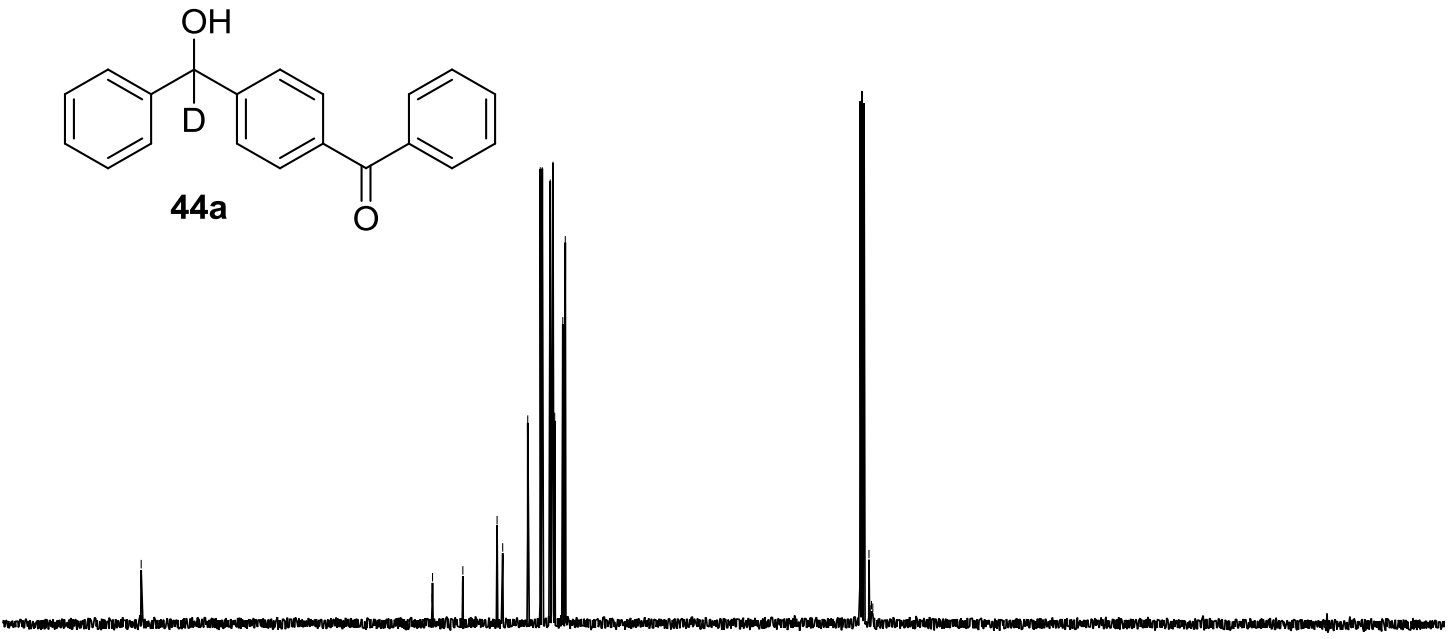

$\begin{array}{lllllllllllllllllllll}210 & 200 & 190 & 180 & 170 & 160 & 150 & 140 & 130 & \begin{array}{c}120 \\ \text { Chemical shift (ppm) }\end{array} & 110 & 100 & 90 & 80 & 50 & 40 & 30 & 20 & 10 & 0 & -10\end{array}$

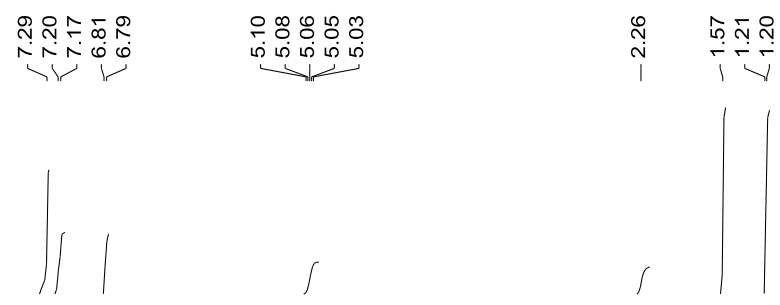<smiles>[R]C(C)(c1ccc(Cl)cc1)c1ccc(OC(C)(C)C(=O)OC(C)C)cc1</smiles>

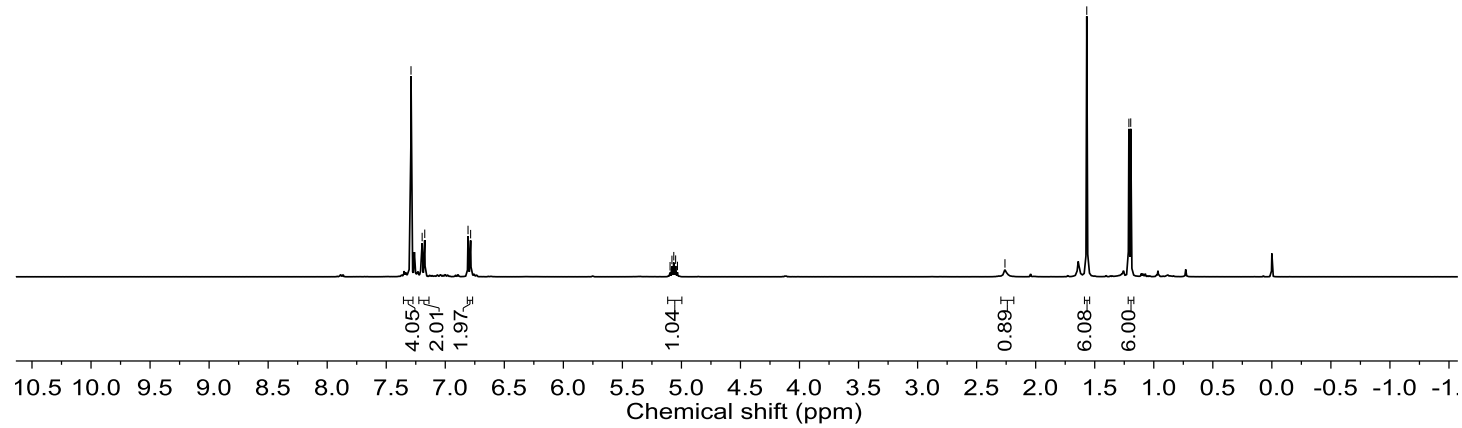


<smiles>[R]C(C)(c1ccc(Cl)cc1)c1ccc(OC(C)(C)C(=O)OC(C)C)cc1</smiles>

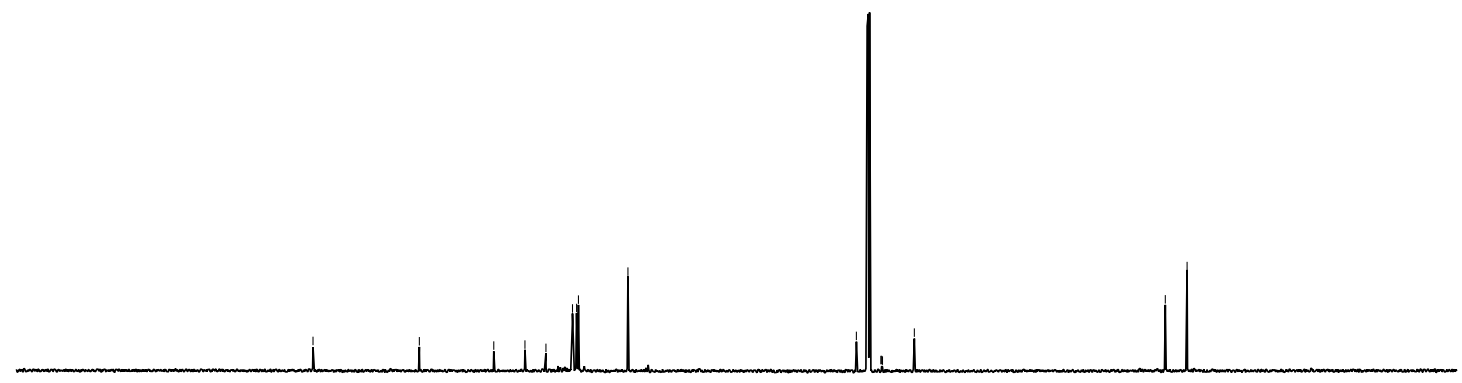

$\begin{array}{llllllllllllllllllllllllll}220 & 210 & 200 & 190 & 180 & 170 & 160 & 150 & 140 & 130 & 120 & 110 & 100 & 90 & 80 & 70 & 60 & 50 & 40 & 30 & 20 & 10 & 0 & -10 & -20\end{array}$ Chemical shift (ppm)

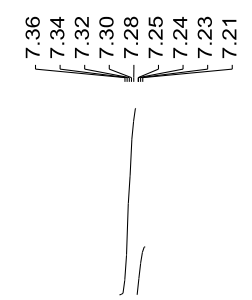<smiles>[2H]C(OCCN(C)C)(c1ccccc1)c1ccccc1</smiles>

$46 a$

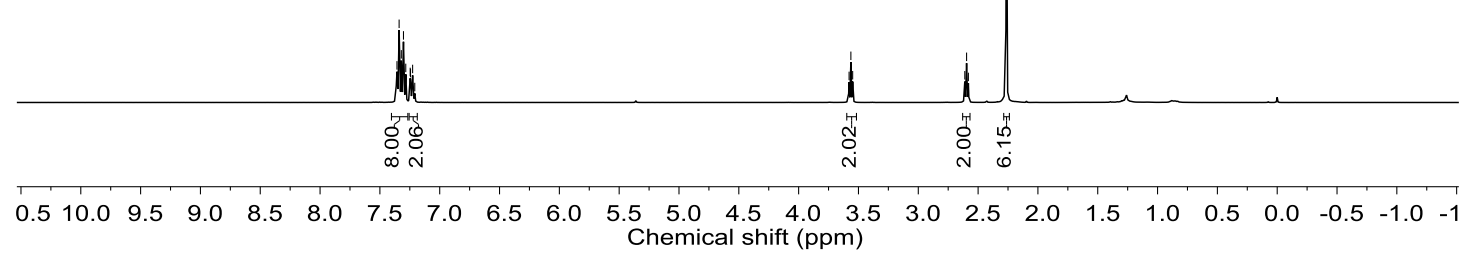


iv

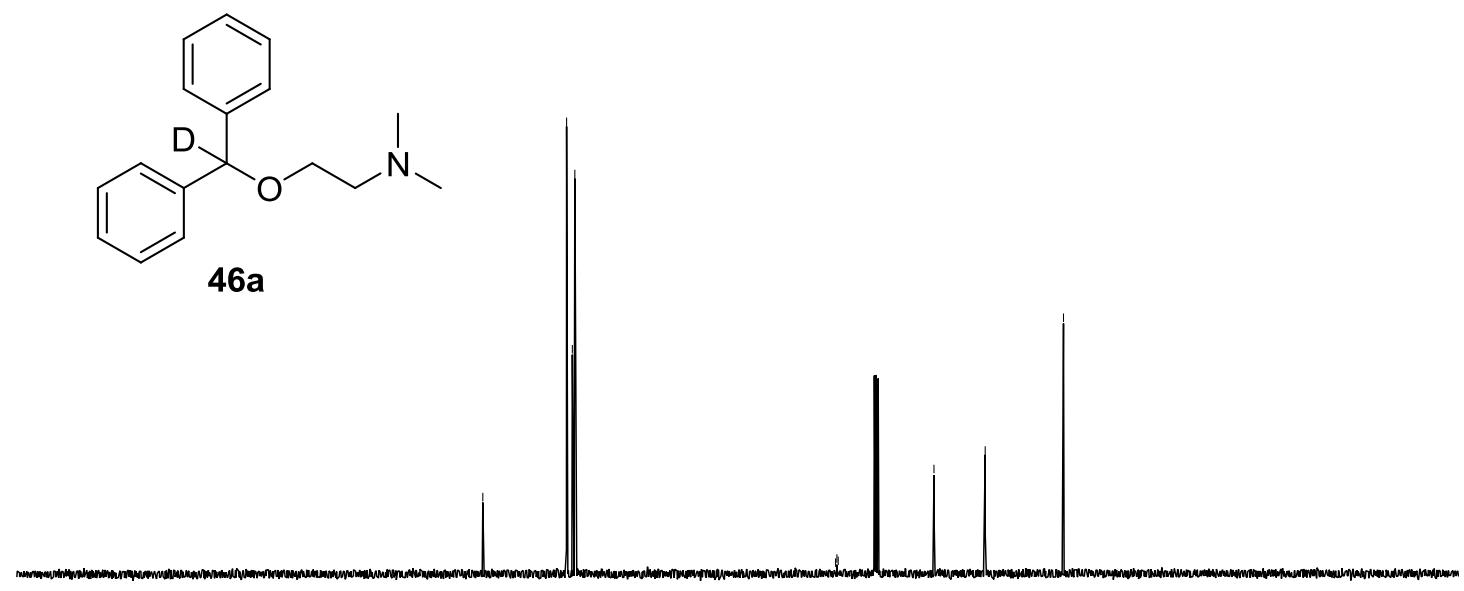

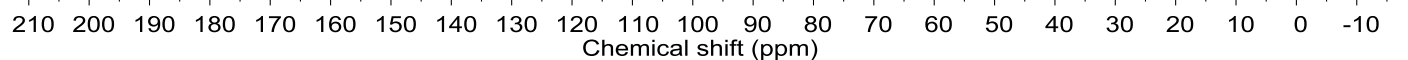

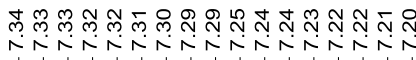

$\|$

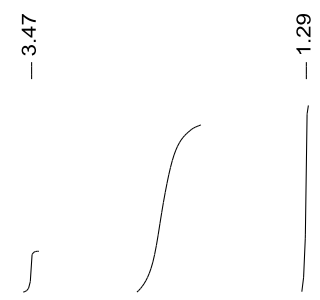<smiles>[2H]C(c1ccccc1)(c1ccc(Cl)cc1)N1CCN(Cc2ccc(C(C)(C)C)cc2)CC1</smiles>

48a

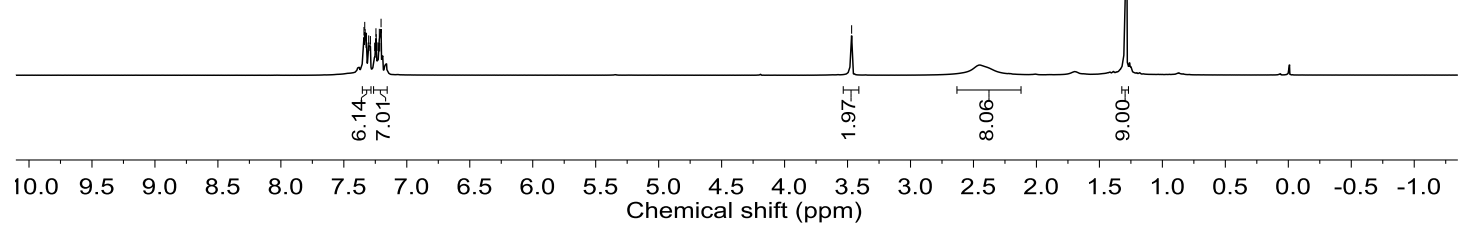


<smiles>[2H]C(c1ccccc1)(c1ccc(Cl)cc1)N1CCN(Cc2ccc(C(C)(C)C)cc2)CC1</smiles>

$48 a$

$\begin{array}{llllllllllllll}220 & 210 & 200 & 190 & 180 & 170 & 160 & 150 & 140 & 130 & 120 & 110 & 100 & 90\end{array}$ Chemical shift (ppm)
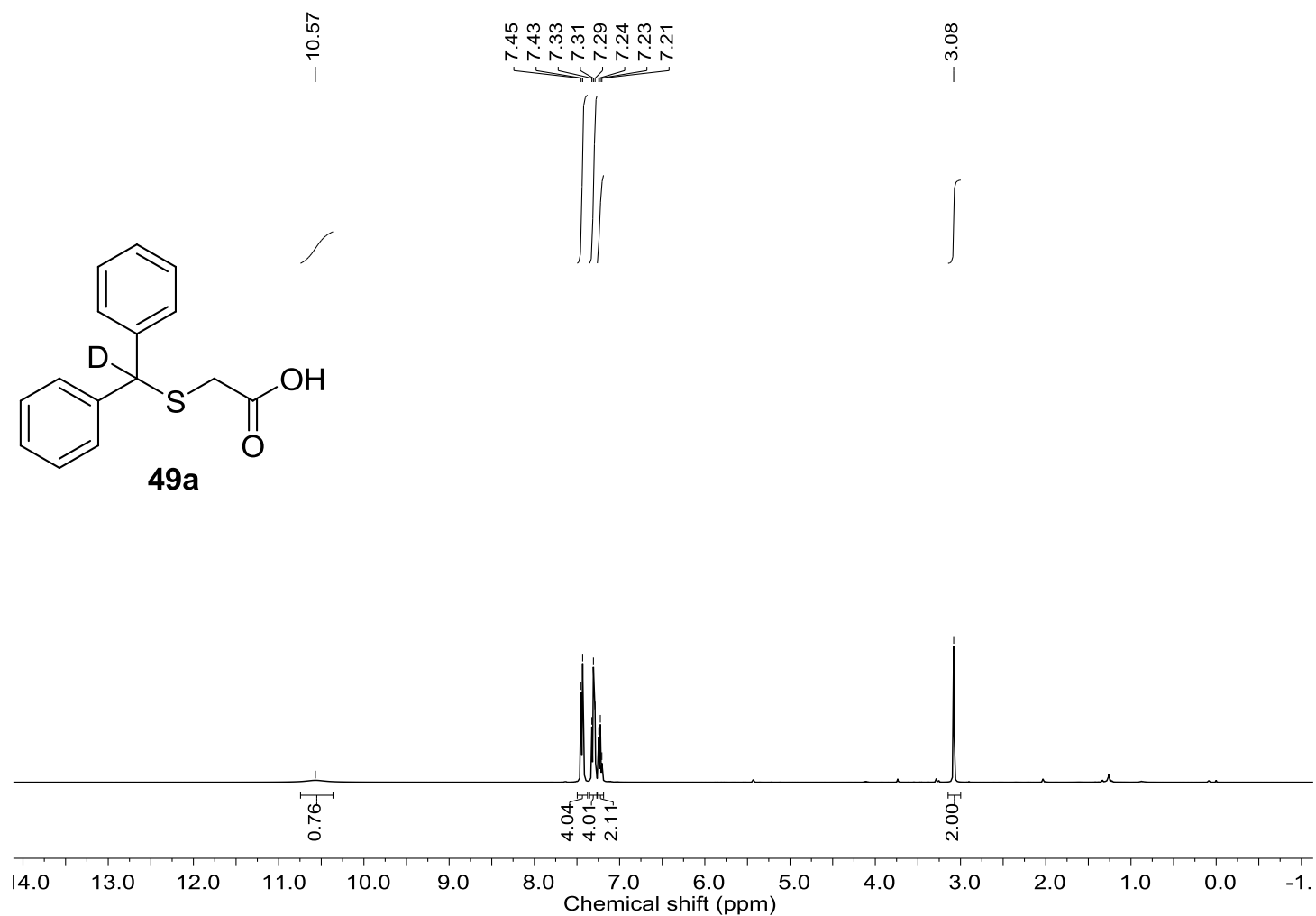


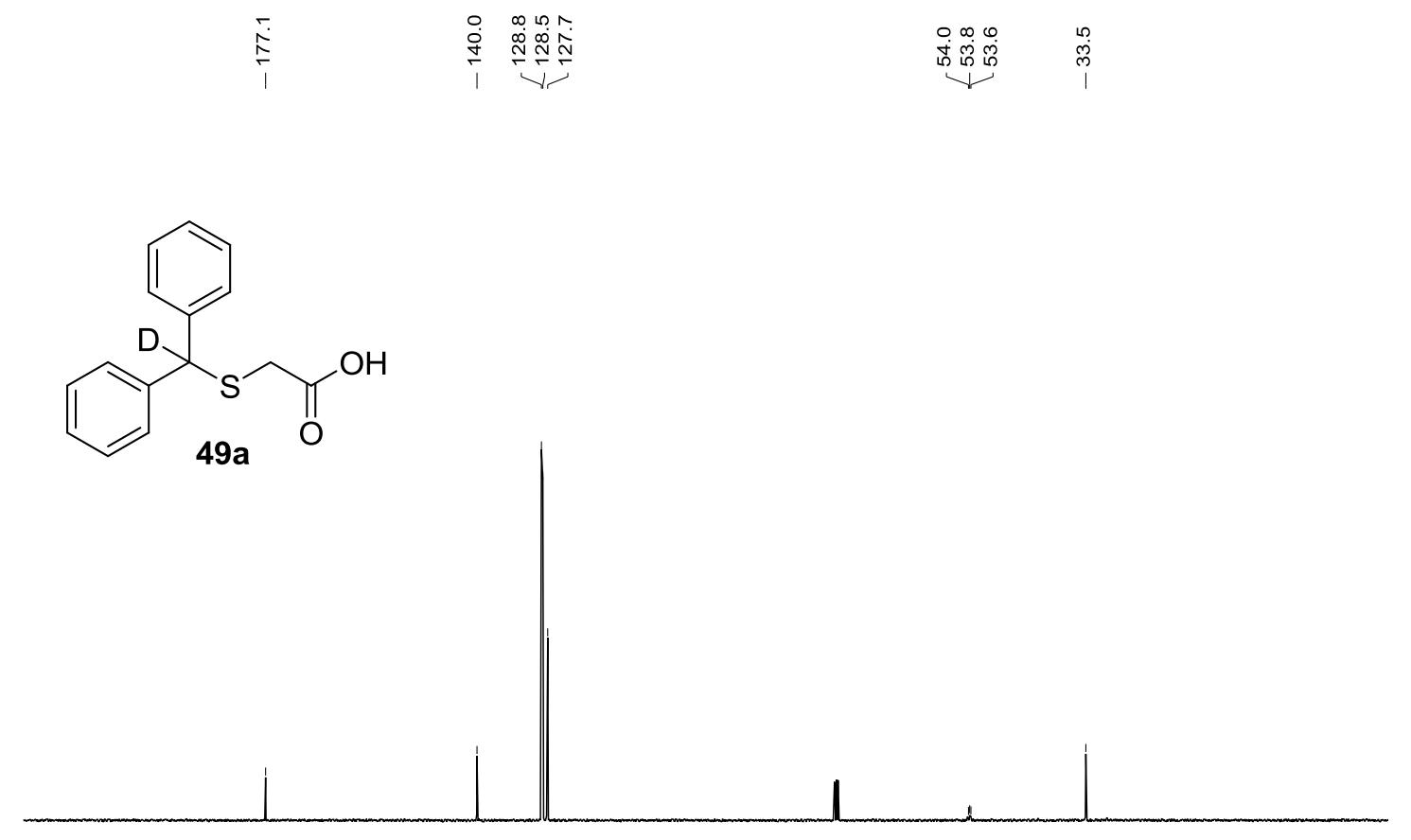

$\begin{array}{llllllllllllllllllllllllllllllll}210 & 200 & 190 & 180 & 170 & 160 & 150 & 140 & 130 & 120 & 110 & 100 & 90 & 80 & 70 & 60 & 50 & 40 & 30 & 20 & 10 & 0 & -10\end{array}$ Chemical Shift (ppm) 


\section{References}

1. Piomelli, D. B., T; Bertozzi, Fabio; et al., Carbamate Derivatives of Lactam Based N-Acylethanolamine Acid Amidase (NAAA) Inhibitors. WO2014/144836 [P]. 2014.

2. Williams, C. M.; Johnson, J. B.; Rovis, T., Nickel-Catalyzed Reductive Carboxylation of Styrenes Using CO2. J. Am. Chem. Soc. 2008, 130 (45), 14936-14937.

3. Liu, G.; Xu, B., Hydrogen bond donor solvents enabled metal and halogen-free Friedel-Crafts acylations with virtually no waste stream. Tetrahedron Lett. 2018, 59 (10), 869-872. 